\title{
Spherical Model for Turbulence
}

\author{
Thesis by \\ Chung-Yu Mou \\ In Partial Fulfillment of the Requirements \\ for the Degree of \\ Doctor of Philosophy
}

California Institute of Technology

Pasadena, California

1993

(Defended May 19, 1993) 


\section{Acknowledgments}

First and foremost, I would like to express my deepest gratitude to my advisor Professor Peter Weichman, who contributed essential ideas for the writing and the content of this thesis. I want to thank him for his generous support and inspiring guidance throughout my formative years at Caltech. Dr. Weichman taught me how to think about problems in more physical terms even though sometimes the problem itself may be mathematically oriented. From him, I have also learned the importance of being persistent in doing research. If not for this persistence, a number of bottlenecks in this work would not have been gone through. He has taught me the most valuable lessons I have ever learned.

I want to thank Professor Michael Cross, who has had a great influence on me. Listening to him always makes me discover something I have not thought about before.

A special note of thanks is due to Patricia Stevens, who makes me feel at home in Sloan Annex. I am very grateful to Eugenia Kuo for providing me with valuable advice and being a good friend, and to Dr. Peter Day for carefully proof-reading this thesis. I also thank Dr. Yuhai Tu for inspiring discussions.

I owe my wife Wen-Jean and my parents my deepest gratitude. Without their support and encouragement, this work would not have been possible. 


\section{Abstract}

A new set of models for homogeneous, isotropic turbulence is considered in which the Navier-Stokes equations for incompressible fluid flow are generalized to a set of $N$ coupled equations in $N$ velocity fields. It is argued that in order to be useful these models must embody a new group of symmetries, and a general formalism is laid out for their construction. The work is motivated by similar techniques that have had extraordinary success in improving the theoretical understanding of equilibrium phase transitions in condensed matter systems. The key result is that these models simplify when $N$ is large. The so-called spherical limit, $N \rightarrow \infty$, can be solved exactly, yielding a closed pair of nonlinear integral equations for the response and correlation functions. These equations, known as Kraichnan's Direct Interaction Approximation (DIA) equations, are, for the first time, solved fully in the scale-invariant turbulent regime, and the implications of these solutions for real turbulence $(N=1)$ are discussed. In particular, it is argued that previously applied renormalization group techniques, based on an expansion in the exponent, $y$, that characterizes the driving spectrum, are incorrect, and that the Kolmogorov exponent $\zeta$ has a nontrivial dependence on $N$, with $\zeta(N \rightarrow \infty)=\frac{3}{2}$. This value is remarkably close to the experimental result, $\zeta \approx \frac{5}{3}$, which must therefore result from higher order corrections in powers of $\frac{1}{N}$. Prospects for calculating these corrections are briefly discussed: though daunting, such a calculations would, for the first time, provide a controlled perturbation expansion for the Kolmogorov, and other, exponents. Our techniques may also be applied 
to other nonequilibrium dynamical problems, such as the KPZ equation for interface growth, and perhaps to turbulence in nonlinear wave systems. 


\section{Contents}

1 Introduction 1

1.1 The Energy Cascade . . . . . . . . . . . . . . 1

1.2 The Kolmogorov Argument . . . . . . . . . . . . . 4

1.3 Renormalization Group Approach . . . . . . . . . . . . 6

1.4 Analogy to Spin Models with Long-range Interactions . . . . . . . . 13

1.5 The $\frac{1}{N}$-expansion and the Spherical Limit . . . . . . . 16

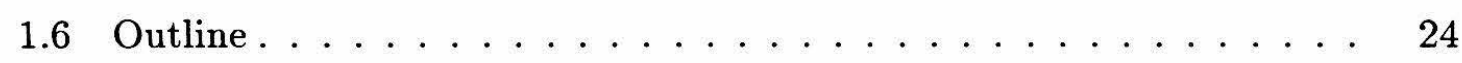

2 Generalization to N velocity fields $\quad 25$

2.1 Analogy to Spin Models . . . . . . . . . . . . . . . 25

2.2 Diagramatic Formalism . . . . . . . . . . . . . . . 28

2.3 Symmetries of $\stackrel{\leftrightarrow}{A}_{N} \ldots \ldots \ldots \ldots \ldots \ldots \ldots$

2.4 Group Theoretical Considerations . . . . . . . . . . . . 38

2.4 .1 Trace Invariants $\ldots \ldots \ldots \ldots \ldots \ldots \ldots$

2.4 .2 Wigner Coefficients ................ 43

2.5 Graphical Considerations . . . . . . . . . . . . . . 48

2.6 Galilean Invariance $\ldots \ldots \ldots \ldots$ 
3 The Spherical Limit $(N \rightarrow \infty) \quad 53$

3.1 Graph Decomposition Rules . . . . . . . . . . . . . . . . 53

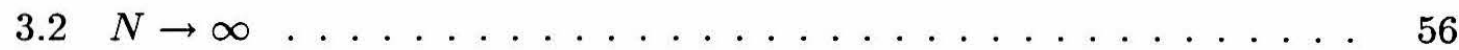

3.3 The Resulting Equations $\ldots \ldots \ldots$. . . . . . . . . . . . . 61

4 Scaling Properties of the Spherical Limit 65

4.1 Recovery of the RG's Results and the Analysis of Convergence . . . 65

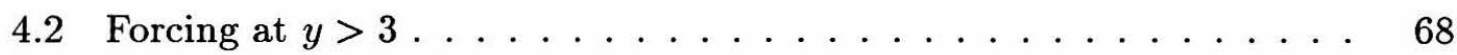

4.3 Viscous Cutoff in the Spherical Limit . . . . . . . . . . . . . . 75

5 Summary and Future Work $\quad 84$

A The Ward Identity and the Renormalization of $\lambda_{0} \quad 88$

A.1 The Ward Identity $(N=1) \ldots \ldots \ldots \ldots \ldots$

A.2 Direct Graph Analysis $\ldots \ldots \ldots . \ldots \ldots$

$\begin{array}{ll}\text { B The Model DIA Equations } & \mathbf{9 7}\end{array}$

$\begin{array}{ll}\text { C Details of Numerical Work } & 108\end{array}$

$\begin{array}{ll}\text { D Large- } N \text { Limit of a Cubic Invariant } & 115\end{array}$

$\begin{array}{ll}\text { References } & 121\end{array}$ 


\section{List of Figures}

1-1 Phase diagram for magnets with long range interactions . . . . . . . 14

1-2 Contrast between two results: the thick lines are exponents in the spherical limit, while the thin lines are those obtained from $y_{0}=y_{c}=4$

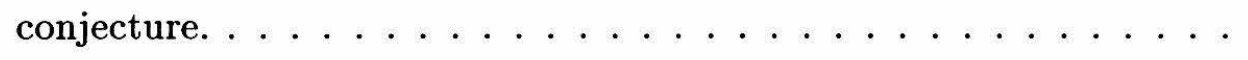

1-3 Turbulence phase diagram ................. 20

2-1 (a) $\hat{G}_{0}(\mathbf{k}, \omega) \hat{\tau}_{\alpha \beta}, \hat{v}_{\alpha}$ is represented by the straight line, $i \hat{w}_{\beta}$ is represented by the wavy line. (b) $\hat{U}_{0}(\mathbf{k}, \omega) \hat{\tau}_{\alpha \beta}\left(\right.$ c) $\frac{i}{2} \lambda_{0} P_{\alpha \beta \gamma}(\mathbf{k}) \ldots . . . .34$

$2-2$ (a) $\hat{G}_{0} \hat{\tau}_{\alpha \beta} \delta_{m}^{n}$ (b) $\hat{U}_{0} \hat{\tau}_{\alpha \beta} \delta_{m}^{n}$ (c) $\frac{i}{2} \lambda_{0} P_{\alpha \beta \gamma} A_{l m n}^{N} \ldots \ldots . \ldots 36$

3-1 Graph decomposition rules ................. 54

3-2 Lower order terms of two particle irreducible diagrams . . . . . . . 57

3-3 Diagrammatic equations in the spherical limit ......... 61

4-1 Scaling function $u(s)$ for different y's with $u(0)=1 . y$ is 3.1 for the outmost curve and increases 0.1 for each successive curve. . . . . . 76

$4-2 \quad \delta_{0}(y)$ vs. $y \ldots \ldots \ldots \ldots \ldots$

4-3 Turbulent scaling function for various dimensions . . . . . . . 78

4-4 Deviation of the correct solution from Kraichnan's solution . . . . . 79 
viii

A-1 Two possible orientations when the largest time vertex connects to an

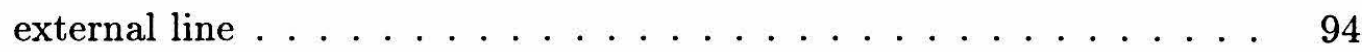

A-2 Three possible orientations when the largest time vertex is internal . $\quad 96$

C-1 Comparison of linear and quadratic interpolations . . . . . . . . 112

C-2 Comparison of two different numbers of divisions . . . . . . . 113

D-1 Two possible configurations: (a) $m \times n$ (b) $n \times m$ (c) An example for the construction of a zero-phase term . . . . . . . . . . 119 


\section{Chapter 1}

\section{Introduction}

\subsection{The Energy Cascade}

Perhaps the most basic issue in the theory of homogeneous turbulence is the nature of the so-called Kolmogorov energy cascade[1]. To describe the problem in the simplest possible terms, consider a three-dimensional fluid that is being stirred on some lengthscale, $l_{0}$, much larger than any dissipative length-scale, $l_{\nu}$. The stirring force causes (kinetic) energy to be input into large-scale, long wavelength hydrodynamic flows. If the fluid equations of motion were linear, the energy would remain in these long wavelength modes for all time. However, the equations are, in fact, nonlinear, and energy will gradually be transferred to shorter and shorter wavelength modes via the interactions between them. Eventually this cascade process will input energy into small-scale modes, of size $l_{\nu}$, which are strongly damped by viscosity. At this point the energy is dissipated irreversibly, and finally appears as heat. A steady state is then achieved in which energy is dissipated at the same rate that it is generated, and there is a kind of momentum-space flux of energy from small wavevectors, $k=O\left(l_{0}^{-1}\right)$, 
to large wavevectors, $k=O\left(l_{\nu}^{-1}\right)$. In the intermediate inertial range, $l_{0}^{-1} \ll k \ll l_{\nu}^{-1}$, the equations of motion are essentially scale invariant, and one expects power-law behavior of the energy spectrum,

$$
E(k) \approx A k^{-\zeta}, \quad l_{0}^{-1} \ll k \ll l_{\nu}^{-1} .
$$

The exponent $\zeta$ is called the Kolmogorov exponent, and, crudely, the question of its value is the fundamental issue in the theory of turbulence.

To state the problem in more formal terms, consider the Navier-Stokes equations for a three-dimensional incompressible fluid:

$$
\frac{\partial \mathbf{v}}{\partial t}+\lambda_{0}(\mathbf{v} \cdot \nabla) \mathbf{v}=-\frac{1}{\rho_{0}} \nabla p+\nu_{0} \nabla^{2} \mathbf{v}+\mathbf{f} ; \quad \nabla \cdot \mathbf{v}=0
$$

where $\mathbf{v}(\mathbf{r}, t)$ is the velocity field, $p(\mathbf{r}, t)$ is the pressure (determined completely by the incompressibility condition), $\mathbf{f}(\mathbf{r}, t)$ is the external driving force (without loss of generality, we take $\nabla \cdot \mathbf{f}=0), \rho_{0}$ is the mass density, $\nu_{0}$ is the kinematic viscosity, and the coupling constant, $\lambda_{0}$, physically equal to unity, is included for convenience. Since we assume the stirring to be large-scale, the Fourier amplitudes, $\hat{\mathbf{f}}(\mathbf{k}, \omega)$, of the driving force vanish rapidly for $k \gg m_{0} \equiv l_{0}^{-1}$. In the absence of the nonlinear convective term, $\lambda_{0}(\mathbf{v} \cdot \nabla) \mathbf{v}$, we would have

$$
\hat{\mathbf{v}}(\mathbf{k}, \omega)=\hat{\mathbf{f}}(\mathbf{k}, \omega) /\left(i \omega+\nu_{0} k^{2}\right)
$$


and only those velocity Fourier modes, $\hat{\mathbf{v}}(\mathbf{k}, \omega)$, for which $k \lesssim m_{0}$ would be substantially excited, and no small-scale motions would result. However, the nonlinearity leads to interactions between modes, and energy will gradually be transferred to shorter wavelengths.

We may estimate the lengthscale, $l_{\nu}$, at which the viscosity becomes important using dimensional analysis[1]. If energy is input into the system at a rate $\bar{\epsilon}$ per unit mass, and is more-or-less conserved in the inertial range, then it must be dissipated at the same rate at the length scale $l_{\nu}$. The only viscosity dependent quantity (with the correct dimensions of length) intrinsic to the dissipation process is

$$
l_{\nu} \equiv \frac{1}{\Lambda}=\left(\nu_{0}^{3} / \bar{\epsilon}\right)^{\frac{1}{4}}
$$

A well defined inertial range clearly requires some combination of small viscosity, large energy input, and large stirring length.

The energy spectrum is obtained from the velocity-velocity correlation function,

$$
U\left(\mathbf{r}-\mathbf{r}^{\prime}, t-t^{\prime}\right)=\frac{1}{2}\left\langle\mathbf{v}(\mathbf{r}, t) \cdot \mathbf{v}\left(\mathbf{r}^{\prime}, t^{\prime}\right)\right\rangle
$$

with Fourier transform

$$
\hat{U}(\mathbf{k}, \omega)=\int d^{d} r d t e^{i(\mathbf{k} \cdot \mathbf{r}+\omega t)} U(\mathbf{r}, t) .
$$


The meaning of the average, $\langle\cdot\rangle$, will be made clear below. The (angular integrated) energy spectrum is then defined by

$$
E(k)=B_{d} k^{d-1} \int_{-\infty}^{\infty} \frac{d \omega}{2 \pi} \hat{U}(\mathbf{k}, \omega)
$$

where the angular factor $B_{d}=(d-1) \pi^{\frac{1}{2} d} / \Gamma\left(\frac{1}{2} d\right)$ is chosen so that $E=\int_{0}^{\infty} d k E(k)=$ $\frac{1}{2}\left\langle\mathbf{v}^{2}\right\rangle$ is the total energy per unit mass, and for later convenience we have kept the spatial dimension, $d$, as a free parameter. In the inertial range, the power law form (1.1) is expected to hold.

\subsection{The Kolmogorov Argument}

In 1941, Kolmogorov[1] presented a simple argument for the value of $\zeta$. The argument was based on two fundamental assumptions. First, the cascade process was assumed to be local: in a sense, to be made precise later, the fluid equations lead mainly to exchanges of energy between modes with wavenumbers of the same order of magnitude. This allows one to define a momentum-space energy flux, which is the rate at which energy is transferred "through" wavevectors of magnitude $k$. Locality postulates that this flux is independent of $k$ in the inertial range, and must therefore be precisely equal to $\bar{\epsilon}$. Second, the energy spectrum was assumed to be independent of the length scales $l_{0}$ and $l_{\nu}$. This turns out to be the more questionable assumption. It basically postulates that as the stirring length, $l_{0}$, diverges, with $\bar{\epsilon}$ fixed, the 
energy spectrum at any given fixed $k$ in the inertial range remains unchanged. The larger scale motions therefore do not affect the details of the local cascade process. Through simple dimensional analysis these two assumptions together determine $E(k)$ : the unique combination of $\bar{\epsilon}$, and $k$ that yields a quantity with the same dimensions as $E(k)$ is

$$
E(k) \approx C_{K} \bar{\epsilon}^{\frac{2}{3}} k^{-\frac{5}{3}}, \quad m_{0} \ll k \ll \Lambda
$$

independently of the dimension, $d$. The dimensionless Kolmogorov constant, $C_{K}$, is postulated to be a universal number (for given $d$ ).

There seem to be two schools of thought on the validity of (1.8). The Kolmogorov prediction, and its derivation, would probably not receive the attention it does today if it did not fit the experimental data so well[2]. One school takes this agreement as strong evidence that $\zeta=\frac{5}{3}$ is exact, and this has led to numerous attempts, based to varying degrees on the actual fluid equations themselves[3,4], to put the result (1.7) on a firmer theoretical footing. Unfortunately, all of these derivations contain uncontrolled approximations, and the inherent danger is that they may all simply be more complicated rephrasings of Kolmogorov's original argument.

The second school (which includes the present authors) takes the view that turbulence is a strongly interacting, nonlinear problem, and that it would be very surprising (if not disappointing!) if the answer were indeed so simple. Given the failure of all attempts to date to prove its exactness, the proximity of experimental reality to the $\frac{5}{3}$-law should tentatively be viewed as coincidental[5], and some systematic means 
sought to distinguish $\zeta$ from $\frac{5}{3}$.

It is simple enough to parameterize such a distinction. If we relax the condition that $E(k)$ be independent of the outer scale, $l_{0}$, the energy spectrum may then depend on the dimensionless combination $k l_{0}=k / m_{0}$, and equation (1.8) may be generalized to

$$
E(k)=C_{K}^{\prime} \bar{\epsilon}^{\frac{2}{3}} k^{-\frac{5}{3}}\left(k / m_{0}\right)^{-\mu / 9},
$$

and thus $\zeta=\frac{5}{3}+\frac{\mu}{9}$. This definition of the exponent $\mu$ seems standard in the literature, originating from the Kolmogorov-Obukhov-Yaglom log-normal theory[2], in which $\mu$ is proportional to the variance of $\frac{\partial}{\partial k} \ln [\epsilon(k)]$, where $\epsilon(k)$ is the (fluctuating) energy flux at scale $k$ (no longer equal to the constant $\bar{\epsilon}$ ). Experimentally one finds $\mu \approx 0.2-0.5$. The exponent $\mu$ may also be interpreted in terms of the fractal codimension, $d-D_{f}$, of the dissipation region via $\mu=3\left(d-D_{f}\right)$.

\subsection{Renormalization Group Approach}

The most modern approach to the theory of turbulence is based on renormalization group ideas[4]. The renormalization group method has proven extraordinarily successful in the treatment of strongly interacting, highly nonlinear problems in equilibrium statistical mechanics. One might hope that the method would be equally successful in treating the problem of turbulence, and hence resolve the differences between the two schools of thought. This hope turns out to be unfounded, as we shall detail 
below. However, the method does yield exact results for related problems, which can then serve as a basis for comparison with appropriate limiting cases of a more general theory. For this reason we summarize the renormalization group results in fair detail. In applying the renormalization group method to turbulence, one begins by modelling the stirring force, $\mathbf{f}$, as a stochastic variable, usually taken to be Gaussian with zero mean and Fourier transformed variance

$$
\left\langle\hat{f}_{i}(\mathbf{k}, \omega) \hat{f}_{i}\left(\mathbf{k}^{\prime}, \omega^{\prime}\right)\right\rangle=D(\mathbf{k}, \omega) \hat{\tau}_{i j}(\mathbf{k}) \delta\left(\mathbf{k}+\mathbf{k}^{\prime}\right) \delta\left(\omega+\omega^{\prime}\right),
$$

where $\hat{\tau}_{i j}(\mathbf{k})=\delta_{i j}-k_{i} k_{j} / k^{2}$ is the transverse projection operator arising from the choice $\nabla \cdot \mathbf{f}=0$. The $\delta$-functions reflect the basic assumption that the turbulence is homogeneous. Within this model, true turbulence is obtained when the driving spectrum, $D(\mathbf{k}, \omega)$, vanishes rapidly for $k \gg m_{0}$. One may well question whether this model yields the same Kolmogorov spectrum as one with a more deterministic stirring force, i.e., whether or not they lie in the same "universality class." Clearly for a very weak deterministic force, the flows will also be deterministic. However, as the strength of the forcing grows, the onset of turbulence is expected to occur through various routes to temporal chaos. Eventually (through as yet ill-understood means), as the driving strength increases, flows that are both temporally and spatially chaotic are generated[6]. Once the flows are chaotic, the behavior in the inertial range is expected to be insensitive to the detailed structure of the forcing, and the stochastic model is probably appropriate. This question will not be addressed any further in 
this work; from now on we simply work with the model (1.10). The meaning of the average in (1.5) is now clear: the velocity field is to be averaged over all realizations of the stochastic driving force.

In addition to the velocity correlator, $\hat{U}(\mathbf{k}, \omega)$, there is another crucial two-point correlation function, namely the response function, $\hat{G}(\mathbf{k}, \omega)$, which measures the average response of the velocity field to an infinitesimal forcing field:

$$
\left\langle\frac{\delta \hat{v}_{i}(\mathbf{k}, \omega)}{\delta \hat{f}_{j}\left(\mathbf{k}^{\prime}, \omega^{\prime}\right)}\right\rangle=\hat{G}(\mathbf{k}, \omega) \hat{\tau}_{i j}(\mathbf{k}) \delta\left(\mathbf{k}+\mathbf{k}^{\prime}\right) \delta\left(\omega+\omega^{\prime}\right)
$$

and with the Gaussian stochastic driving, (1.10), one has the more explicit relation

$$
\left\langle\hat{v}_{i}(\mathbf{k}, \omega) \hat{f}_{j}\left(\mathbf{k}^{\prime}, \omega^{\prime}\right)\right\rangle=\hat{G}(\mathbf{k}, \omega) D(\mathbf{k}, \omega) \hat{\tau}_{i j}(\mathbf{k}) \delta\left(\mathbf{k}+\mathbf{k}^{\prime}\right) \delta\left(\omega+\omega^{\prime}\right) .
$$

The response function is causal, so that in the time domain $\hat{G}(\mathbf{k}, t)=0$ for $t<0$, while

$$
\hat{G}\left(\mathbf{k}, t \rightarrow 0^{+}\right)=1 \text { for all } \mathbf{k} \text {. }
$$

The renormalization group method is based on a form of the driving spectrum that has completely opposite characteristics from that required for turbulence. Specifically, the driving spectrum is assumed to grow stronger as $k$ increases:

$$
D(\mathbf{k}, \omega) \equiv D(k)=\frac{D_{0} k^{4-d}}{\left(k^{2}+m_{0}^{2}\right)^{\frac{1}{2} y}}
$$


where the parameter $y$ is assumed to be either negative, or positive but small.

When $y=2-d, D(k) \approx D_{0} k^{2}$ and the model is that of a thermally driven fluid (for this case it is safe to take $m_{0} \equiv 0$ )[4]. The fluctuation-dissipation theorem then requires that $D_{0}=k_{B} T \nu_{0}$, and the relation $\hat{U}(\mathbf{k}, \omega)=\left(2 D_{0} / \nu_{0}\right) \operatorname{Re} \hat{G}(\mathbf{k}, \omega)$ holds. The model was originally proposed by Forster, Nelson, and Stephen[4a] in order to study the effects of small-scale thermal fluctuations on large-scale hydrodynamics. By using a momentum-shell renormalization group technique, in which short length-scale fluctuations are successively integrated out, these authors were able to derive recursion relations for the length-scale dependent effective viscosity, $\nu(l)$, and the effective nonlinearity coefficient, $\bar{\lambda}(l)$, where $\bar{\lambda}(l)=\lambda(l)\left[D(l) / \nu(l)^{3}\right]^{1 / 2}$ and $l$ is the renormalization group flow parameter. For $d>2$ they showed that $\lim _{l \rightarrow \infty} \nu(l)=\nu_{R}$ is finite, while $\lim _{l \rightarrow \infty} \bar{\lambda}(l)=0$, indicating that linear hydrodynamics, with a renormalized (eddy) viscosity, $\nu_{R}$, appropriately describes large-scale flows. Generally, $\nu_{R}$ is larger than $\nu_{0}$ (and is, in fact, positive even when $\nu_{0} \equiv 0$ ), indicating enhanced diffusive transport by small-scale eddies. The energy spectrum obeys the equipartition principle, $E(k) \sim k^{d-1}$. In contrast, for $d<2$ nontrivial large-scale behavior results: In an expansion in $y=2-d, \bar{\lambda}(l)$ flows to a finite fixed point value, $\bar{\lambda}_{R}=O(y)$. However, the energy spectrum still obeys $E(k) \sim k^{d-1}$, a consequence of the fluctuation-dissipation theorem.

These authors also considered the case $y=4-d$, and hence $D(k) \approx D_{0}$, in which all wavenumbers are driven equally ("uniform" driving). In this case, linear 
hydrodynamics is valid on large scales only when $d>4$. Once more, when $y=4-d>$ $0, \bar{\lambda}(l)$ flows to a nontrivial fixed-point value, $\bar{\lambda}_{R}=O(y)$, and $E(k) \sim k^{\frac{1}{3}(2 d-5)}$. Note that in both cases the borderline between the two different kinds of behavior occurs at $y=0$.

A short time later, DeDominicis and Martin[4b] formalized and generalized these results using field-theoretic methods. From Ward identities, and the general form (1.14) for the driving spectrum, they showed that for $y<0$ linear hydrodynamics results on large scales, while for $y>0, \bar{\lambda}_{R}=O(y)$ is finite. Furthermore, they showed that to all orders in $y$ (see Appendix A for a discussion), the energy spectrum takes the power-law form

$$
E(k) \sim k^{-\zeta(y)}, \zeta(y)=1-\frac{2}{3} y,
$$

with no further explicit dimensionality dependence in $\zeta$.

The renormalization group picture implies much more than power-law energy spectra. The existence of fixed points implies scaling of the correlation functions. Thus, for example, in the inertial range the correlation and response functions are predicted to take the forms

$$
\begin{aligned}
& \hat{G}(\mathbf{k}, \omega) \approx A_{1} k^{-z} g\left(\omega / \tilde{\nu} k^{z}\right) \\
& \hat{U}(\mathbf{k}, \omega) \approx A_{2} k^{-\Delta} u\left(\omega / \tilde{\nu} k^{z}\right), k, \omega \rightarrow 0
\end{aligned}
$$

where the exponents $\Delta$ and $z$, and the scaling functions $g(s)$ and $u(s)$ are universal, 
while $A_{1}, A_{2}$ and $\tilde{\nu}$ are nonuniversal scale factors. The dynamical exponent $z$ provides a connection between length-scales and time-scales. The fact that it appears also in the prefactor of (1.16) is a consequence of the normalization (1.13). DeDominicis and Martin show that, again to all orders in $y$, the exponents are given by

$$
z=2-\frac{1}{3} y ; \quad \Delta=d+\frac{1}{3} y \quad(y>0) .
$$

Notice that this implies the "hyperscaling" relation,

$$
\Delta+z=d+2
$$

which will be significant later on. The relation (1.7) gives $E(k) \approx B_{d} \bar{u}_{0} \tilde{\nu} k^{-\zeta(\Delta, z)}$, where

$$
\zeta(\Delta, z)=\Delta-z-d+1
$$

and $\bar{u}_{0}=\int_{-\infty}^{\infty} \frac{d s}{2 \pi} u(s)$. Together with (1.18) this immediately yields the result (1.15). It should be emphasized that (1.20) is a general scaling relation, whereas (1.18) and (1.19) are valid only within the $y$-expansion.

Now, what connection, if any, do these results have with turbulence? Clearly, what we will call the "short-ranged" driving problem, in which $D(k)$ effectively vanishes for $k \geq m_{0}$, corresponds, in some sense, to the limit $y \rightarrow \infty$ of the "long-range" driving problem. If we blindly take this limit in (1.15), $\zeta$ diverges to plus infinity, which is clearly nonsensical. This is our first hint that the $y$-expansion must have a 
finite radius of convergence, $y_{0}$.

From theories of critical phenomena one knows that "input" exponents, like $y$, need not actually be infinite to recover "short-range" behavior. Rather, for sufficiently large values, $y>y_{c}$, one expects long-range driving, (1.14), to become technically irrelevant, and give rise only to lower-order corrections to the leading short-range (i.e., turbulent) behavior. In the simplest, most optimistic scenario, the value of $y$ at which this happens is precisely the radius of convergence of the expansion around $y=0$, i.e., $y_{c}=y_{0}$. In the renormalization group picture, this corresponds to a continuous coalescence of the long-range fixed point with the short-range one, and the exponents correspondingly go continuously over to their short-range values. We would conclude, in this case, that $\zeta=\frac{2}{3} y_{c}-1, z=2-\frac{1}{3} y_{c}$, and $\Delta=d-2+\frac{1}{3} y_{c}$.

Unfortunately, within the $y$-expansion there is no direct way of ascertaining either $y_{0}$ or $y_{c}$. DeDominicis and Martin[4b] have shown that for $y>4$ there are an infinite number of relevant Galilean invariant perturbations to the linear hydrodynamical fixed point. This says nothing about the stability of the power-law driven fixed point. In particular, it neither establishes that $y_{0}=4$, nor that $y_{0}=y_{c}$, though a great deal of work has been based on precisely these assumptions[7]. What makes them so compelling is that, as first noticed by DeDominicis and Martin[4], they yield precisely the Kolmogorov result for the energy spectrum, $\zeta=\frac{5}{3}$. 


\subsection{Analogy to Spin Models with Long-range}

\section{Interactions}

In order to place the renormalization group results in a clearer context, it is useful to consider the following analogy[8]. Consider the standard ferromagnetic phase transition in an Ising model with long-range interactions. The Hamiltonian is

$$
H_{I}=-\frac{1}{2} \sum_{i \neq j} J_{i j} s_{i} s_{j}
$$

where $s_{i}= \pm 1$ is the Ising spin at $d$-dimensional lattice site $i$, and the exchange constants have the power-law behavior

$$
J_{i j}=J_{0}\left|\mathbf{R}_{i j}\right|^{-(d+\sigma)}, \quad i \neq j, J_{0}>0,
$$

in contrast to those for the standard Ising model which vanish when $i$ and $j$ are not nearest neighbors. In Figure 1-1 we show the boundaries between various types of critical behavior in the $d-\sigma$ plane. For $\sigma<\frac{1}{2} d$ or $d>4$ a Gaussian model controls the critical behavior. For sufficiently large $\sigma$ and $d<4$ the usual short-range critical behavior, characteristic of the nearest neighbor Ising model, results. For $d<4$ there is an intermediate range of $\sigma$ for which nontrivial long-range critical behavior results. We make an analogy between Gaussian behavior in the spin model, and linear hydrodynamic behavior in the fluid model; between short-range Ising critical 


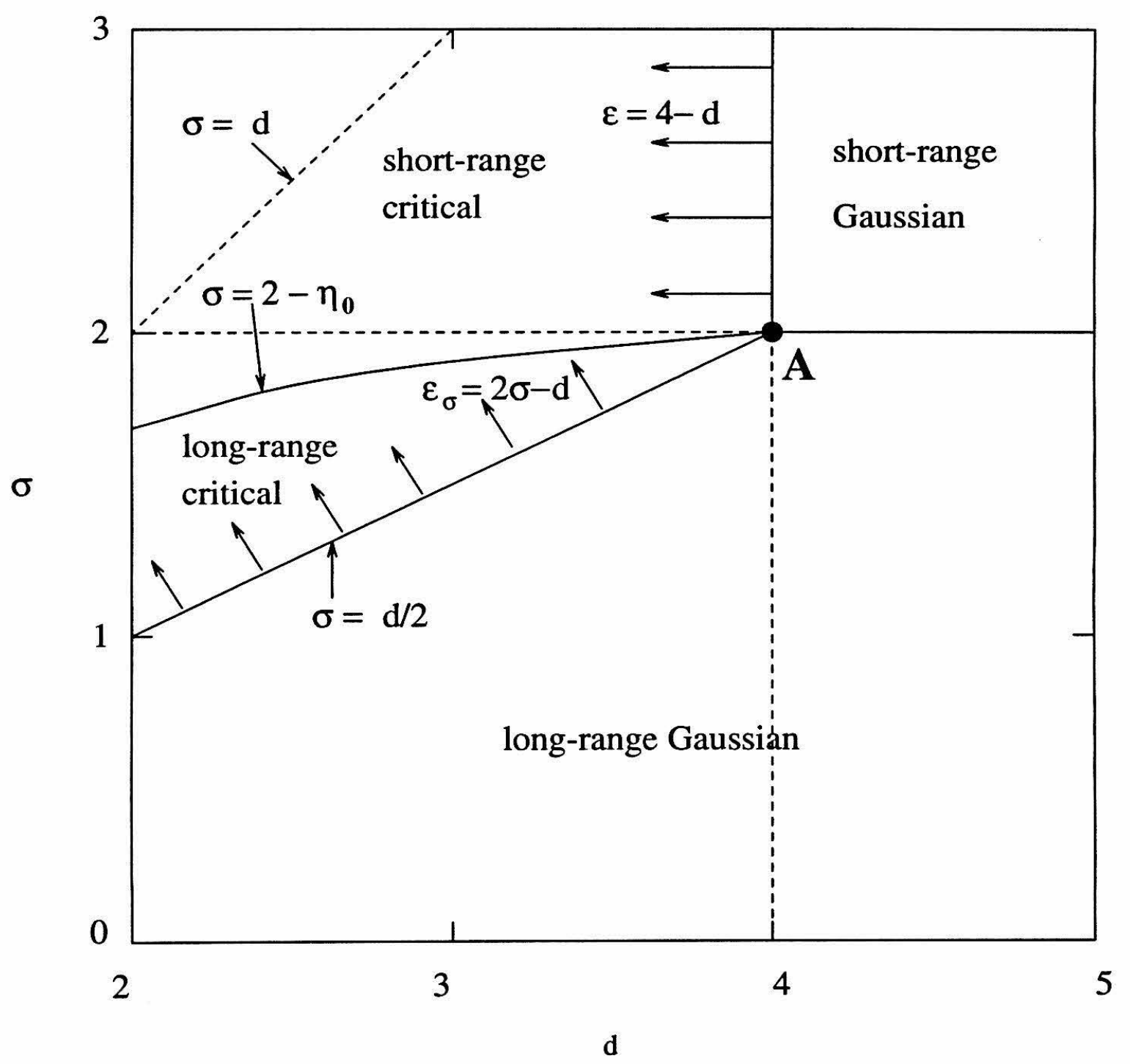

Figure 1-1: Phase diagram for magnets with long range interactions 
behavior and real turbulence; and between nontrivial long-range critical behavior and the long-range driven fluid. Analogous to the $y$-expansion in the fluid problem is the $\epsilon_{\sigma}$-expansion[9], with $\epsilon_{\sigma}=2 \sigma-d$, which penetrates upwards into the long-range critical region from the line $\sigma=\frac{1}{2} d$. In addition, an analysis equivalent to that of DeDominicis and Martin[4b] shows that for $\sigma>d$, there are infinitely many relevant perturbations to the long-range Gaussian fixed point (corresponding to multicritical behavior of all orders). It is clear from Figure 1-1 that this line has no significance whatsoever so long as $d>2$.

In the spin problem one has the advantage that the short-range critical behavior may be accessed directly through the usual $\epsilon$-expansion[10] about $d=4$. Thus one can check directly the relevance of long-range interactions at the short-range Ising fixed point. This, as well as more general arguments, allow one to fix precisely the boundary between short-range and long-range critical behavior[11], which occurs when $\sigma=2-\eta_{0}$, where $\eta_{0}(d)$ is the short-range value of the critical decay exponent, $\eta$. In addition, the long-range value of $\eta$ is given exactly by $\eta_{L R}=2-\sigma$, much like the exact results (1.15) and (1.18) for the fluid problem. Note that this immediately implies continuity of $\eta$ across the boundary.

We may now address, by analogy, the question of the position of the equivalent boundary in the fluid problem. There is no information in the $\epsilon_{\sigma}$-expansion about the value of $\eta_{0}$, and therefore no hint that the value $\sigma=2-\eta_{0}$ is special. Only by locating both fixed points, and seeing when they merge, or equivalently, seeing when the long- 
range fixed point becomes unstable to the short-range one, can this boundary be located. Naively one might have expected this boundary to occur at $\sigma=2$, for this is when the $k^{2}$ and $k^{\sigma}$ terms in the Fourier transform, $\hat{J}(\mathbf{k})=\hat{J}_{0}+\hat{J}_{2} k^{2}+\hat{J}_{\sigma} k^{\sigma}+O\left(k^{4}\right)$, of $J_{i j}$ exchange dominance as $k \rightarrow 0$. For subtle reasons, involving the nontrivial rescaling of the $k^{2}$ term under renormalization[11], this expectation is false. There is no reason not to have similar doubts about the $y_{0}=y_{c}=4$ conjecture in the turbulence problem.

It is basically the existence of the point $A$ in Figure 1-1, near which all of the four possible fixed points are simultaneously perturbatively accessible (both $\epsilon$ and $\epsilon_{\sigma}$ are small) that allows one to infer the detailed characteristics of the short-range-longrange boundary. The apparent absence of such a point in the $d-y$ plane for fluids is what leads to the failure of the renormalization group method in turbulence. We are therefore forced to seek a new approach in order to make progress on this problem.

\subsection{The $\frac{1}{N}$-expansion and the Spherical Limit}

In the theory of equilibrium phase transitions there are actually two analytic techniques that have provided many of the fundamental insights into the nature of critical phenomena: the epsilon expansion $[9,10]$ and the $\frac{1}{N}$-expansion[12]. The first, as we have seen, corresponds most closely to the $y$-expansion, and is based on the fact that the critical behavior is simple in sufficiently high dimension, $d>d_{c}$. One can then perform a systematic expansion in $\epsilon=d_{c}-d$ when $d<d_{c}$ (here $d_{c}=4$ for the 
short-range Ising model, and $d_{c}=2 \sigma$ for the long-range Ising model with $\sigma<2$ ).

The second technique involves analytically continuing the problem to one with a larger number of degrees of freedom, $N$. Thus the Ising Hamiltonian is generalized to the $O(N)$ model, $H^{(N)}=-\frac{1}{2} \sum_{i \neq j} J_{i j} \mathbf{s}_{i} \cdot \mathbf{s}_{j}$, where $\mathbf{s}_{i}$ is an $N$-component unit vector, $\left|\mathbf{s}_{i}\right|=1$. If taken in an appropriate fashion, the so-called spherical limit, $N \rightarrow \infty$, is often analytically tractable, and a systematic expansion in $\frac{1}{N}$ may be developed for the exponents[12]. The epsilon expansion has generally proven the more definitive of the two in understanding critical phenomena, mainly because it transpires that the dimensionality of interest, namely $d=3$, is usually, in some sense, closer to $d_{c}=4$ than are physical values of $N$, say $N=1,2$, or 3 , to $N=\infty$. However, the $\frac{1}{N}$ expansion has the advantage that the dimensionality, $d$, is a completely free variable, and is therefore useful in the study of physics in lower dimensions where $\epsilon$ is not small.

In turbulence, as described, the analogue of the epsilon expansion is uncontrolled in the region of interest. We seek, therefore, an approach in which the variable $y$ [or, more generally, the entire driving function $D(\mathbf{k}, \omega)$ ], like the dimensionality, $d$, in the spin problem, may be taken as a free parameter. This thesis, then, is concerned with the construction of a $\frac{1}{N}$-expansion for turbulence[13]. Our primary aim is to obtain an analytically tractable spherical limit and then to elucidate the dependence of the Kolmogorov spectrum, (1.1), on $y$. In particular, we wish to understand the analyticity properties of $\zeta(y)$, and how true turbulence is recovered in the limit of 
large $y$.

The essential results of our study are summarized below, and in Figures 1-2 and 1-3. The spherical limit yields a pair of coupled, nonlinear integral equations for the functions $\hat{G}(\mathbf{k}, \omega)$ and $\hat{U}(\mathbf{k}, \omega)$ [see equations (3.3) and (3.4) in Chapter 3] which turn out to be precisely Kraichnan's Direct Interaction Approximation (DIA) equations[14]. Although they have been around for nearly 35 years, these equations have never been fully solved. Kraichnan[14], through a series of scaling arguments, concluded that

$$
z=1, \Delta=d+\frac{3}{2}, \text { and } \zeta=\frac{3}{2}
$$

for short-range driving. We shall show that these results are, in fact, correct and present complete solutions for the scaling functions $g(s)$ and $u(s)$ (see Chapter 4). Note that these exponent values violate the hyperscaling relation (1.19) and therefore do not correspond to any value of $y$.

How do these results come about? If one now examines the solutions to the DIA equations in the presence of long-range driving, one discovers some amazing things. Firstly, so long as the integrals converge in the scaling limit, $m_{0} \rightarrow 0$, one finds precisely the hyperscaling relation (1.19). If, furthermore, it is assumed that $D(k)$ controls the scaling, then (1.18) and (1.15) hold and the $y$-expansion results are reproduced exactly. In fact, it can be shown that the DIA equations are an exact resummation of the $O(y)$ renormalization group recursion relations. The limit $N \rightarrow \infty$ is therefore exact to $O(y)$. However, the DIA equations also extend these 


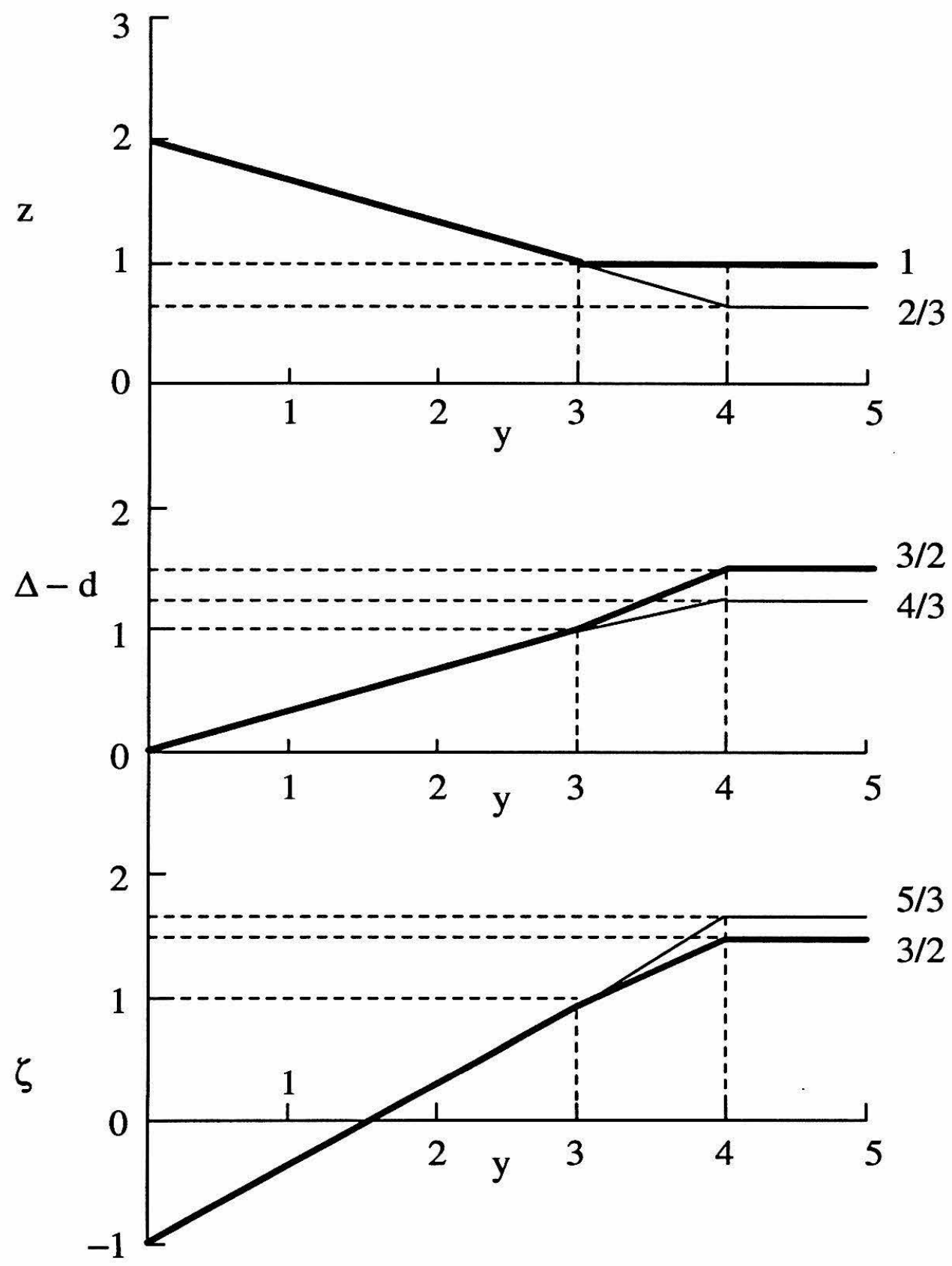

Figure 1-2: Contrast between two results: the thick lines are exponents in the spherical limit, while the thin lines are those obtained from $y_{0}=y_{c}=4$ conjecture. 


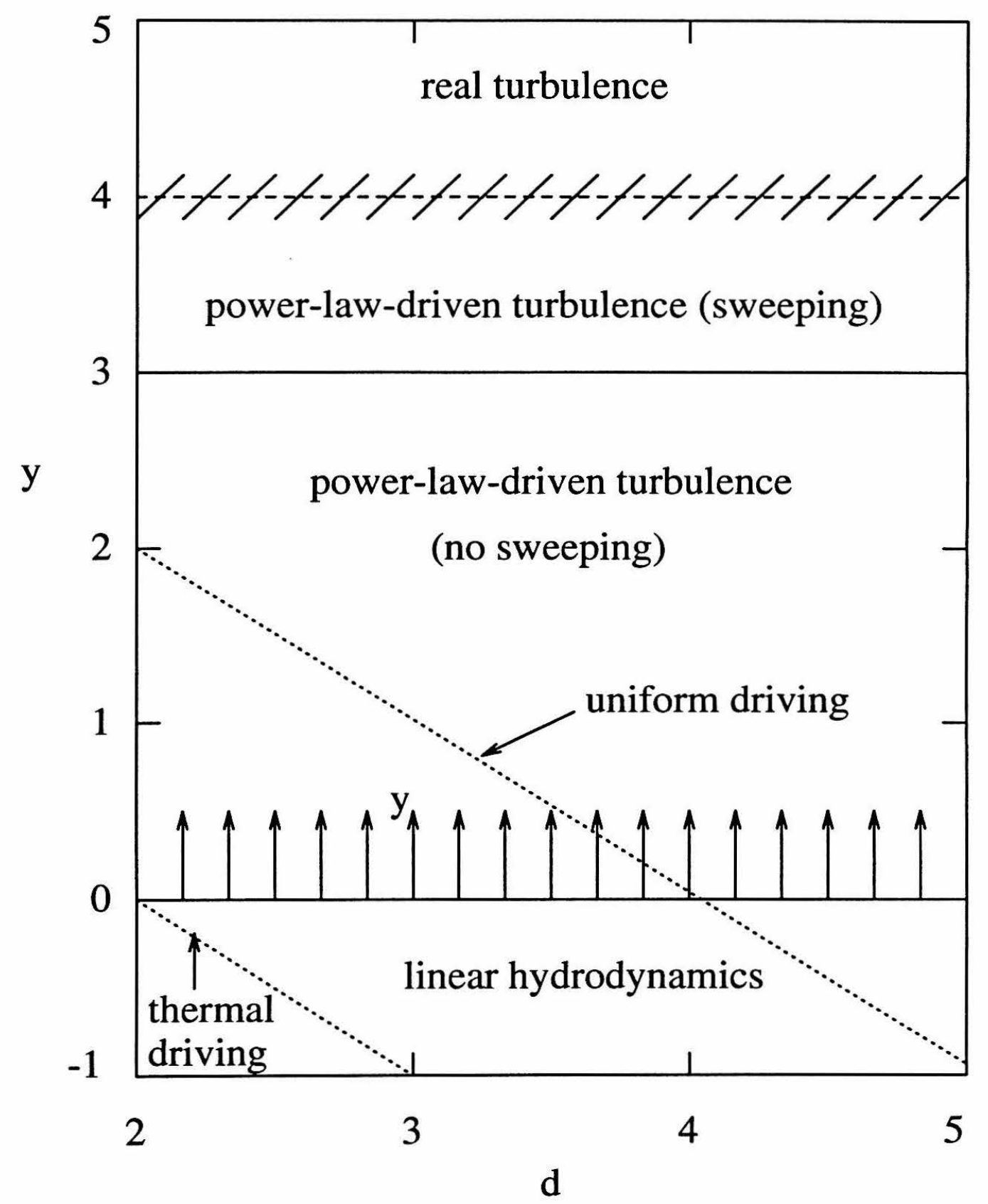

Figure 1-3: Turbulence phase diagram 
recursion relations to arbitrary driving, $D(\mathbf{k}, \omega)$, and allow one to see where the renormalization group results break down. Specifically, when $y \geq 3$ the DIA integrals no longer converge, and the limit $m_{0} \rightarrow 0$ becomes subtle. By careful asymptotic analysis one can show that the dynamical exponent sticks at $z=1$, and, again, so long as the driving still controls the scaling, one finds

$$
\Delta=d+1+\frac{y-3}{2} \text { and } \zeta=1+\frac{y-3}{2} \text {. }
$$

Finally, to connect these with the Kraichnan $\zeta=\frac{3}{2}$ result, one must determine the value of $y$ at which the driving ceases to control the scaling. This occurs at $y=4$ : for $y>4$ long-range driving becomes technically irrelevant, and except for lower order corrections to scaling, is equivalent to the real turbulence problem. For $y>4$ all exponents then stick at values determined by (1.24) with $y=4$, i.e., precisely the values (1.23) predicted by Kraichnan[14].

In Firgure 1-2 these results for the exponents in the spherical limit are contrasted with those obtained from the $y_{0}=y_{c}=4$ conjecture. In Firgure 1-3 we show a plot analogous to that for the Ising model, Firgure 1-1. Our basic prediction is then that a new type of "critical behavior" intervenes between the boundary of convergence for the $y$-expansion, $y_{0}=3$ (which we believe to be exact: see below), and the onset of true turbulence at $y_{c}=4$ (which will likely have corrections for finite $N$ ). Thus, although real turbulence indeed occurs for $y>y_{c}=4$, the different behavior in the intermediate interval, $3<y<4$, changes completely the values of the turbulent 
exponents. In renormalization group language, a new stable fixed point bifurcates away from the now unstable long-range driving fixed point, and only later coalesces with the true turbulence fixed point. This scenario is clearly even more involved than that for the Ising model, where the analogues, $\sigma_{0}$ and $\sigma_{c}$, of $y_{0}$ and $y_{c}$, though nontrivial, are at least equal, $\sigma_{0}=\sigma_{c}=2-\eta_{0}$.

The physics behind the $y=3$ borderline is, in fact, well known, corresponding to the oft-quoted effects of sweeping of smaller eddies by larger ones. The dynamical exponent value $z=1$ then confirms the Taylor "frozen in" hypothesis: small-scale turbulent structures are swept past a fixed observer at a speed which fluctuates, but remains more-or-less constant in order of magnitude. This speed basically determines the shortest time-scale in the problem, and the small scale structures change very little in the time it takes them to be swept by. Thus, measuring the temporal velocity fluctuations at a single point is nearly equivalent to measuring spatial velocity fluctuations along a one-dimensional line at a single time. Therefore, inertial range frequency spectra and wavenumber spectra should be the same up to a rescaling factor which depends on the large-scale cutoff, $m_{0}$. The fact that the spherical model equations reflect this physics is heartening and leads us to believe that $y_{0}=3$ and $z=1$ are exact results.

In a nutshell, it is attempts to remove the boundary at $y=3$ that motivates many of the attempts to show that the Kolmogorov $\frac{5}{3}$-law is exact (see especially Ref. 15). At the level of the DIA equations, these efforts focus on producing, in some natural 
way, extra terms which cancel the divergent parts of the integrals when $y \geq 3$. One problem with this is that if these same terms are produced when $y<3$, there will be large- $k$ divergences in the region described (presumably correctly) by the $y$-expansion. The theory will then fail to encompass the known exact results.

The second problem is connected with the entire philosophy of the large- $N$ approach. The limit $N \rightarrow \infty$ produces an exactly soluble model. Any alterations in this model can come only from finite- $N$ corrections; the DIA equations, not their subtracted versions, are fundamental. The differences between the value $\zeta=\frac{3}{2}$ at $N \rightarrow \infty$ and the experimental result $\zeta \approx \frac{5}{3}$ at $N=1$ are now accounted for in a very natural way: we propose that, just as for the $O(N)$ spin model, the exponents $\Delta$ and $\zeta$ (and most likely the boundary $y_{c}$ ) vary continuously with $N$, interpolating between $\zeta(\infty)=\frac{3}{2}$ and $\zeta(1) \approx \frac{5}{3}$. The fact that $\zeta(\infty) \neq \frac{5}{3}$ is strong evidence that $\zeta(1)$ is a nontrivial exponent, not obtainable through any simple argument. A real test of our approach would be to compute the first correction, in powers of $\frac{1}{N}$, to $\zeta(\infty)$. As will be seen in later chapters, this is a daunting task, but seems to be a necessary step in order to confirm our ideas.

We therefore view the DIA $\frac{3}{2}$-result not as a problem to be fixed, but as an amazingly accurate zeroth order result, differing from the experimental result by only 10\%. Large- $N$ expansions for spin models seldom do this well! 


\subsection{Outline}

The remainder of this thesis is organized as follows: In Chapter 2 we generalize the incompressible Navier-Stokes equations, (1.2), to $N$ equations for $N d$-dimensional velocity fields. In Chapter 3 we obtain the limit $N \rightarrow \infty$ and derive the spherical/DIA equations. In Chapter 4 we solve these equations and derive the results shown in Figures 1-2 and 1-3. Finally in Chapter 5 we summarize and describe work for the future. Appendix A is devoted to the derivation of the Ward identity. In Appendix B we describe a time-independent toy model whose large- $\mathrm{N}$ limit yields frequency-independent DIA-type equations that can be solved analytically using power laws. The solution to these toy equations contain all the structure of their frequency-dependent counterparts, and provide some further insight into the workings of the full DIA equations. Appendix C outlines the numerical work that is involved in Chapter 4. In Appendix D, we describe one of our attempts at generalizing our large- $N$ model. Although this attempt is fruitless, it provides another example for the application of the formalism developed in Chapter 3. 


\section{Chapter 2}

\section{Generalization to $\mathrm{N}$ velocity fields}

\subsection{Analogy to Spin Models}

The most straightforward generalization of the Navier-Stokes equations, (1.2), to $N$ velocity fields, $\mathbf{v}^{l}, l=1, \ldots, N$, is

$$
\frac{\partial \mathbf{v}^{l}}{\partial t}+\lambda_{0} \sum_{m, n=1}^{N} A_{N}^{l m n}\left(\mathbf{v}_{m} \cdot \nabla\right) \mathbf{v}_{n}=-\frac{1}{\rho_{0}} \nabla p^{l}+\nu_{0} \nabla^{2} \mathbf{v}^{l}+\mathbf{f}^{l} ; \nabla \cdot \mathbf{v}^{l}=0, l=1, \ldots, N
$$

where $\mathbf{f}^{l}$ are independent random forces. The only question one must address is that of the choice of the tensor $\overleftrightarrow{A}_{N}$ (for later convenience, a distinction, to be defined below, has been made between upper and lower latin indices).

Here we again appeal to the spin model analogy. The generalization of the Ising Hamiltonian, (1.21), to $N$-component spins, $\mathbf{s}_{i}=\left(s_{i, 1}, \ldots, s_{i, N}\right)$, is

$$
H^{(N)}=-\frac{1}{2} \sum_{i \neq j} J_{i j} \sum_{l, m=1}^{N} C_{N}^{l m} s_{i, l} s_{j, m},\left|\mathbf{s}_{i}\right|^{2}=N
$$

where one must choose an appropriate $N \times N$ positive definite matrix $\stackrel{\leftrightarrow}{C}_{N}$ (the normal- 
ization $\left|\mathbf{s}_{i}\right|^{2}=N$ is chosen by convention, and yields the correct large- and small- $N$ limits). Since $\overleftrightarrow{C}_{N}$ is symmetric one may perform a rotation in spin-space to diagonalize it, obtaining

$$
H^{(N)}=-\frac{1}{2} \sum_{i \neq j} J_{i j} \sum_{l=1}^{N} \lambda^{l} s_{i, l} s_{j, l},\left|\mathbf{s}_{i}\right|^{2}=N
$$

where $\lambda^{l}$ are the eigenvalues of $\overleftrightarrow{C}_{N}$. Clearly, any state with long-range magnetic order will energetically prefer to align along the component of $\mathbf{s}$ with largest eigenvalue, say $\lambda^{1}$. If $\lambda^{1}$ is unique one can, in fact, show that the critical behavior is completely dominated by this "easy axis," [16] and lies in the same universality class as that of the Ising model, (1.21). We have therefore gained nothing by giving $\mathbf{s}_{i}$ extra components. Only if $\lambda^{1}$ is not unique does the critical behavior change. Thus if $\lambda^{1}=\lambda^{2}=\ldots=\lambda^{M}, M \leq N$, are the largest eigenvalues, then the model has $O(M)$ symmetry and the critical behavior depends on the value of $M$. Again, however, the $N-M$ components with smaller eigenvalues are redundant and do not effect the asymptotic critical behavior. Clearly, then, in order to obtain the simplest possible model, one should take $M=N$ (i.e., $C_{N}^{l m}=\delta_{l m}$ ) and

$$
H^{(N)}=-\frac{1}{2} \sum_{i \neq j} J_{i j} \mathbf{s}_{i} \cdot \mathbf{s}_{j}
$$

This is the so-called $N$-vector model. Special cases are $N=1$, Ising; $N=2, \mathrm{XY}$; and $N=3$, Heisenberg. The crucial property of $H^{(N)}$ is its invariance under the group of 
rotations, $O(N)$, in spin space, and this is what allows the universality class of the transition to vary with $N[17]$.

By analogy, if we seek a generalized model for turbulence in which Kolmogorovtype exponents depend continuously on $N$, it seems likely that one must build into the equations an extra group of symmetries. Thus, by analogy with the set of rotations

$$
s_{i, l}^{\prime}=\sum_{m=1}^{N} R_{N}^{l m}(g) s_{i, m}, g \in O(N), \stackrel{\leftrightarrow}{R}_{N}^{T} \stackrel{\leftrightarrow}{R}_{N}=I_{N}
$$

(here $I_{N}$ is the $N \times N$ identity matrix) which leave $H^{(N)}$ invariant, we seek an $N$ dimensional irreducible representation of a group (technically, a simple compact Lie group), $G$, of transformations along with an appropriate tensor, $\stackrel{A}{A}_{N}$, such that the transformation

$$
\mathbf{v}^{l}=\sum_{m=1}^{N} D_{N}^{l m}(g) \mathbf{v}^{m}, g \in G
$$

leaves the equations of motion (2.1) invariant. Since the group is taken to be compact, the representation may always be taken as unitary, $\stackrel{\leftrightarrow}{D}_{N}^{\dagger} \stackrel{\leftrightarrow}{D}_{N}=I_{N}$. We assume, of course, that the pressures, $p^{l}$, and the forces, $\mathbf{f}^{l}$, also transform under (2.6). We leave open the possibility that the velocity fields are complex (their real and imaginary parts then being the physical variables). The distinction between upper and lower indices is then made: $\mathbf{v}_{l}$ transforms via the inverse (or complex conjugate) representation,

$$
\mathbf{v}_{l}^{\prime}=\sum_{m=1}^{N} \mathbf{v}_{m} D_{N}^{m l}\left(g^{-1}\right)=\sum_{m=1}^{N} \mathbf{v}_{m}\left[\overleftrightarrow{D}_{N}(g)^{-1}\right]^{m l}
$$


If the representation is unitary (which we assume unless stated otherwise), then $\mathbf{v}_{l} \equiv \mathbf{v}^{l *}$, and $D_{N}^{m l}\left(g^{-1}\right)=D_{N}^{l m}(g)^{*}[18]$. If the representation is orthogonal (and hence all quantities are real) there is no distinction between upper and lower indices. If the representation is not unitary, it differs from unitary only by a similarity transformation, and $\mathbf{v}_{l}$ is then an appropriate linear combination of the $\mathbf{v}^{m *}$. Substituting (2.6) and (2.7) into (2.1) we obtain then the condition

$$
A_{N}^{l^{\prime} m^{\prime} n^{\prime}}=\sum_{l, m, n} D_{N}^{l^{\prime \prime} l}(g) D_{N}^{m^{\prime} m}(g) D_{N}^{n^{\prime} n}(g) A_{N}^{l m n}, \forall g \in G
$$

i.e., that $\overleftrightarrow{A}_{N}$ be invariant under the group of transformations $G$. The questions to be addressed then, are, given a group $G$, which irreducible representation should we choose, and given a representation how do we construct appropriate cubic invariants, $\overleftrightarrow{A}_{N} ?$

\subsection{Diagramatic Formalism}

Before addressing these questions it is useful to outline the perturbation theoretic formalism for the Navier-Stokes equations, including its generalization to $N>1$. The formalism was first developed by Martin, Siggia, and Rose[19], extending the earlier Wyld diagrammatic theory[20], and was used by DeDominicis and Martin[4b] in their renormalization group calculations.

First we include the incompressibility condition, $\nabla \cdot \mathbf{v}=0$, explicitly by realizing 
that the gradient of the pressure in (1.2) simply cancels the longitudinal part of the nonlinear term. Thus if we define the $k$-space transverse projection operator,

$$
\hat{\tau}_{\alpha \beta}(\mathbf{k})=\delta_{\alpha \beta}-\frac{k_{\alpha} k_{\beta}}{k^{2}}
$$

and let $\tau_{\alpha \beta}(\mathbf{r})$ be its inverse Fourier transform, then the Navier-Stokes equations may be written

$$
\frac{\partial \mathbf{v}}{\partial t}+\lambda_{0} \stackrel{\leftrightarrow}{\tau} \cdot(\mathbf{v} \cdot \nabla) \mathbf{v}=\nu_{0} \nabla^{2} \mathbf{v}+\mathbf{f}
$$

where we have used the shorthand notation

$$
[\overleftrightarrow{\tau} \cdot(\mathbf{v} \cdot \nabla) \mathbf{v}]_{\alpha}(\mathbf{r})=\sum_{\beta} \int d^{3} r^{\prime} \tau_{\alpha \beta}\left(\mathbf{r}-\mathbf{r}^{\prime}\right) \mathbf{v}\left(\mathbf{r}^{\prime}\right) \cdot \nabla v_{\alpha}\left(\mathbf{r}^{\prime}\right)
$$

Let us define the "Navier-Stokes operator"

$$
\mathbf{N}(\mathbf{r}, t) \equiv \frac{\partial \mathbf{v}}{\partial t}+\lambda_{0} \stackrel{\leftrightarrow}{\tau} \cdot(\mathbf{v} \cdot \nabla) \mathbf{v}-\nu_{0} \nabla^{2} \mathbf{v}
$$

then we may formally compute the statistical average of any functional $F[\mathbf{v}]$ of the velocity field via

$$
\langle F[\mathbf{v}]\rangle=\int D \mathbf{v} F[\mathbf{v}] J[\mathbf{v}]\langle\delta[\mathbf{N}[\mathbf{v}]-\mathbf{f}]\rangle
$$

where $\int D \mathbf{v}$ is a functional integral over all incompressible velocity fields, and is 
defined by an appropriate continuum limit,

$$
\int D \mathbf{v} \equiv \lim _{M \rightarrow \infty} \prod_{i=1}^{M} \int d^{d} v\left(\mathbf{r}_{i}, t_{i}\right) \delta\left(\nabla \cdot \mathbf{v}\left(\mathbf{r}_{i}, t_{i}\right)\right)
$$

where $\left\{\mathbf{r}_{i}, t_{i}\right\}_{i=1}^{M}$ runs over a discrete space-time grid, and $\nabla \cdot \mathbf{v}\left(\mathbf{r}_{i}, t_{i}\right)$ is the obvious discretized divergence at lattice point $\left(\mathbf{r}_{i}, t_{i}\right)$. Similarly,

$$
\delta[\mathbf{N}[\mathbf{v}]-\mathbf{f}]=\lim _{M \rightarrow \infty} \prod_{i=1}^{M} \delta\left(\mathbf{N}\left(\mathbf{r}_{i}, t_{i}\right)-\mathbf{f}\left(\mathbf{r}_{i}, t_{i}\right)\right)
$$

enforces the Navier-Stokes equations at all space-time points. The Jacobian, $J[\mathbf{v}]$, is given by

$$
J[\mathbf{v}]=\operatorname{det}\left[\frac{\delta \mathbf{N}(\mathbf{r}, t)}{\delta \mathbf{v}\left(\mathbf{r}^{\prime}, t^{\prime}\right)}\right]
$$

with the operator

$$
\frac{\delta N_{\alpha}(\mathbf{r}, t)}{\delta v_{\beta}\left(\mathbf{r}^{\prime}, t^{\prime}\right)}=\left[\delta_{\alpha \beta}\left(\partial_{t}-\nu_{0} \nabla^{2}+\lambda_{0} \mathbf{v} \cdot \nabla\right)+\lambda_{0}\left(\partial_{\beta} v_{\alpha}\right)\right] \delta\left(\mathbf{r}-\mathbf{r}^{\prime}\right) \delta\left(t-t^{\prime}\right)
$$

inside the determinant, and is precisely what is needed to convert $\delta\left[\mathbf{v}-\mathbf{N}^{-1}[\mathbf{f}]\right]$ to $\delta[\mathbf{N}[\mathbf{v}]-\mathbf{f}]$.

We now represent the $\delta$-function using the identity $\delta(x)=\int_{-\infty}^{\infty} \frac{d w}{2 \pi} e^{-i w x}$ for each 
space-time point, so that

$$
\langle F[\mathbf{v}]\rangle=\int D \mathbf{v} \int D \mathbf{w} F[\mathbf{v}] e^{-i \int d^{d} r \int d t \mathbf{w}(\mathbf{r}, t) \cdot \mathbf{N}(\mathbf{r}, t)+\ln J[\mathbf{v}]}\left\langle e^{-i \int d^{d} r \int d t \mathbf{w}(\mathbf{r}, t) \cdot \mathbf{f}(\mathbf{r}, t)}\right\rangle,
$$

where $\mathbf{w}(\mathbf{r}, t)$ is also incompressible, i.e., $\nabla \cdot \mathbf{w}=0$. It can be shown that causality (i.e., the fact that $\delta \mathbf{N}(\mathbf{r}, t) / \delta \mathbf{v}\left(\mathbf{r}^{\prime}, t^{\prime}\right)$ vanishes when $\left.t^{\prime}>t\right)$ implies that the Jacobian term reduces to[21]

$$
\ln J[\mathbf{v}]=C_{1}+\int d^{d} r \int d t \sum_{\alpha} \frac{\delta N_{\alpha}(\mathbf{r}, t)}{\delta v_{\alpha}(\mathbf{r}, t)}=C_{1}+\mathbf{C}_{2} \cdot \int d^{d} r \int d t \mathbf{v}(\mathbf{r}, t)
$$

Rotation invariance implies that $\mathbf{C}_{2}$ must vanish, and the Jacobian term is therefore a constant, independent of the velocity field.

Performing the average over the Gaussian random field $\mathbf{f}(\mathbf{r}, t)$, we finally arrive at

$$
\langle F[\mathbf{v}]\rangle=\frac{1}{Z} \int D \mathbf{v} \int D \mathbf{w} F[\mathbf{v}] e^{\mathcal{L}[\mathbf{v}, \mathbf{w}]}
$$

where the Lagrangian is

$$
\begin{aligned}
\mathcal{L}[\mathbf{v}, \mathbf{w}] & =-i \sum_{\alpha, \beta} \int d^{d} r \int d t\left[w_{\alpha}\left(\partial_{t}-\nu_{0} \nabla^{2}\right) v_{\beta} \delta_{\alpha \beta}+\lambda_{0} w_{\alpha} \tau_{\alpha \beta}(\mathbf{v} \cdot \nabla) v_{\beta}\right] \\
& -\frac{1}{2} \sum_{\alpha, \beta} \int d^{d} r \int d t \int d^{d} r^{\prime} \int d t^{\prime} w_{\alpha}(\mathbf{r}, t) D_{\alpha \beta}\left(\mathbf{r}-\mathbf{r}^{\prime}, t-t^{\prime}\right) w_{\beta}\left(\mathbf{r}^{\prime}, t^{\prime}\right)
\end{aligned}
$$


with $D_{\alpha \beta}\left(\mathbf{r}-\mathbf{r}^{\prime}, t-t^{\prime}\right)=\left\langle f_{\alpha}(\mathbf{r}, t) f_{\beta}\left(\mathbf{r}^{\prime}, t^{\prime}\right)\right\rangle[$ see $(1.10)]$, and $Z=\int D \mathbf{v} \int D \mathbf{w} e^{\mathcal{L}[\mathbf{v}, \mathbf{w}]}$ ensures correct normalization by cancelling out the (formally divergent) constant $C_{1}$. One may now extend, in the obvious way, the quantities being averaged to functionals of both $\mathbf{w}$ and $\mathbf{v}$ :

$$
\langle F[\mathbf{v}, \mathbf{w}]\rangle=\frac{1}{Z} \int D \mathbf{v} \int D \mathbf{w} F[\mathbf{v}, \mathbf{w}] e^{\mathcal{L}[\mathbf{v}, \mathbf{w}]} .
$$

This is important as it turns out that response functions may be generated in this way[19]. In particular, for isotropic driving, (1.10), we have

$$
\left\langle i \hat{w}_{\alpha}(\mathbf{k}, \omega) \hat{v}_{\beta}\left(\mathbf{k}^{\prime}, \omega^{\prime}\right)\right\rangle=\hat{G}(\mathbf{k}, \omega) \hat{\tau}_{\alpha \beta}(\mathbf{k}) \delta\left(\mathbf{k}+\mathbf{k}^{\prime}\right) \delta\left(\omega+\omega^{\prime}\right)
$$

[compare (1.11) and (1.12)] while, as before,

$$
\left\langle\hat{v}_{\alpha}(\mathbf{k}, \omega) \hat{v}_{\beta}\left(\mathbf{k}^{\prime}, \omega^{\prime}\right)\right\rangle=\hat{U}(\mathbf{k}, \omega) \hat{\tau}_{\alpha \beta}(\mathbf{k}) \delta\left(\mathbf{k}+\mathbf{k}^{\prime}\right) \delta\left(\omega+\omega^{\prime}\right)
$$

where (1.5) is obtained by realizing that $\operatorname{tr}[\hat{\tau}(\mathbf{k})]=2$. In Fourier space the Lagrangian may be written $\mathcal{L}=\mathcal{L}_{0}+\lambda_{0} \mathcal{L}_{1}$, with

$$
\begin{aligned}
& \mathcal{L}_{0}[\mathbf{v}, \mathbf{w}]=\int_{\mathbf{k}} \int_{\omega}\left[-i\left(-i \omega+\nu_{0} k^{2}\right) \hat{\mathbf{w}}(-\mathbf{k},-\omega) \cdot \hat{\mathbf{v}}(\mathbf{k}, \omega)-\frac{1}{2}|\hat{\mathbf{w}}(\mathbf{k}, \omega)|^{2} D(\mathbf{k}, \omega)\right] \\
& \mathcal{L}_{1}[\mathbf{v}, \mathbf{w}]=-\frac{i}{2} \int_{\mathbf{k}} \int_{\omega} \int_{\mathbf{q}} \int_{\Omega} \sum_{\alpha, \beta, \gamma} P_{\alpha \beta \gamma}(\mathbf{k}) \hat{w}_{\alpha}(-\mathbf{k},-\omega) \hat{v}_{\beta}(\mathbf{k}-\mathbf{q}, \omega-\Omega) \hat{v}_{\gamma}(\mathbf{q}, \Omega)
\end{aligned}
$$


where $P_{\alpha \beta \gamma}(\mathbf{k})=\hat{\tau}_{\alpha \beta}(\mathbf{k}) k_{\gamma}+\hat{\tau}_{\alpha \gamma}(\mathbf{k}) k_{\beta}$, and we have used the short hand notation $\int_{\mathbf{k}} \equiv \int \frac{d^{d} k}{(2 \pi)^{d}}, \int_{\omega} \equiv \int \frac{d \omega}{2 \pi}$, etc. Recalling that $\mathbf{k} \cdot \hat{\mathbf{v}}(\mathbf{k}, \omega)=\mathbf{k} \cdot \hat{\mathbf{w}}(\mathbf{k}, \omega)=0$, we easily compute the zeroth-order $\left(\lambda_{0}=0\right)$ forms

$$
\begin{aligned}
& \hat{G}_{0}(\mathbf{k}, \omega)=\frac{1}{-i \omega+\nu_{0} k^{2}} \\
& \hat{U}_{0}(\mathbf{k}, \omega)=D(\mathbf{k}, \omega)\left|\hat{G}_{0}(\mathbf{k}, \omega)\right|^{2} .
\end{aligned}
$$

The usual diagrammatic perturbation theory[19] in $\lambda_{0}$ results by expanding $e^{\lambda_{0} \mathcal{L}_{1}}$ in a Taylor series and performing the averages term by term. Representing the resulting integrals by Feynman diagrams, the zeroth order correlation function, $\hat{U}_{0}$, becomes a straight line, the zeroth order response function, $\hat{G}_{0}$, becomes a combination straightwavy line. Vertices have three legs (one wavy one and two straight ones) and a momentum-conserving $\delta$-function, along with a factor $\frac{i}{2} \lambda_{0} P_{\alpha \beta \gamma}(\mathbf{k})$ (where $\mathbf{k}$ is the incoming momentum on the wavy leg) accompanies each one (see Figure 2-1).

Generalizing the formalism to $N$ velocity fields is straightforward. Introducing incompressible fields $\mathbf{w}^{l}$ and $\mathbf{w}_{l}, l=1, \ldots, N$ which bear the same relationship to each other as $\mathbf{v}^{l}$ and $\mathbf{v}_{l}$, and assuming

$$
\left\langle f_{\alpha}^{l}(\mathbf{k}, \omega) f_{\beta, m}\left(\mathbf{k}^{\prime}, \omega^{\prime}\right)\right\rangle=D(\mathbf{k}, \omega) \hat{\tau}_{\alpha, \beta}(\mathbf{k}) \delta\left(\mathbf{k}+\mathbf{k}^{\prime}\right) \delta\left(\omega+\omega^{\prime}\right) \delta_{m}^{l}
$$




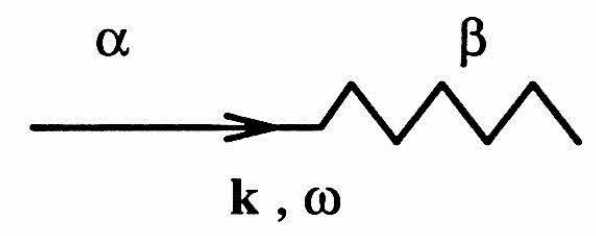

(a)

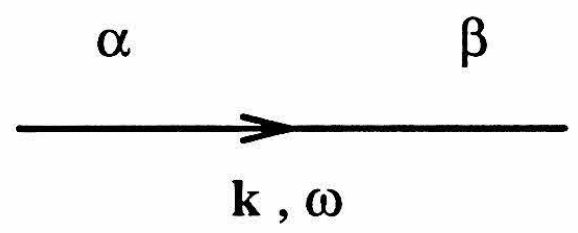

(b)

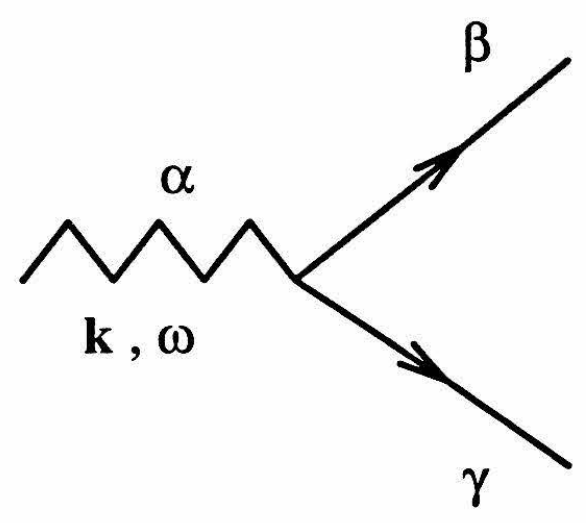

(c)

Figure 2-1: (a) $\hat{G}_{0}(\mathbf{k}, \omega) \hat{\tau}_{\alpha \beta}, \hat{v}_{\alpha}$ is represented by the straight line, $i \hat{w}_{\beta}$ is represented by the wavy line. (b) $\hat{U}_{0}(\mathbf{k}, \omega) \hat{\tau}_{\alpha \beta}$ (c) $\frac{i}{2} \lambda_{0} P_{\alpha \beta \gamma}(\mathbf{k})$ 
one finds a Lagrangian $\mathcal{L}^{(N)}=\mathcal{L}_{0}^{(N)}+\lambda_{0} \mathcal{L}_{1}^{(N)}$, with

$$
\begin{aligned}
\mathcal{L}_{0}^{(N)} & =\sum_{l=1}^{N} \int_{\mathbf{k}} \int_{\omega}\left\{-\frac{i}{2}\left[\left(-i \omega+\nu_{0} k^{2}\right) \hat{\mathbf{w}}_{l}(-\mathbf{k},-\omega) \cdot \hat{\mathbf{v}}^{l}(\mathbf{k}, \omega)+\text { c.c. }\right]\right. \\
& \left.-\frac{1}{2} D(\mathbf{k}, \omega) \hat{\mathbf{w}}^{l}(-\mathbf{k},-\omega) \cdot \hat{\mathbf{w}}_{l}(\mathbf{k}, \omega)\right\} \\
\mathcal{L}_{1}^{(N)} & =-\frac{i}{4} \sum_{l, m, n} \sum_{\alpha, \beta, \gamma} \int_{\mathbf{k}} \int_{\omega} \int_{\mathbf{q}} \int_{\Omega}\left[A_{N}^{l m n} P_{\alpha \beta \gamma}(\mathbf{k}) \hat{\mathbf{w}}_{\alpha, l}(-\mathbf{k},-\omega)\right. \\
& \left.\times \hat{\mathbf{v}}_{\beta, m}(\mathbf{q}-\mathbf{k}, \omega-\Omega) \hat{\mathbf{v}}_{\gamma, n}(\mathbf{q}, \Omega)+\text { c.c. }\right] .
\end{aligned}
$$

The fields $\mathbf{w}^{l}$ are assumed to transform in precisely the same way, (2.6), as the fields $\mathbf{v}^{l}$. The properties (2.7) and (2.8) then immediately imply that both $\mathcal{L}_{0}^{(N)}$ and $\mathcal{L}_{1}^{(N)}$ are invariant under the group, $G_{N}$, of transformations. When $\lambda_{0}=0$ we have

$$
\begin{aligned}
\left\langle i \hat{w}_{\alpha}^{l}(\mathbf{k}, \omega) \hat{v}_{\beta, m}\left(\mathbf{k}^{\prime}, \omega^{\prime}\right)\right\rangle & =\hat{G}(\mathbf{k}, \omega) \hat{\tau}_{\alpha \beta}(\mathbf{k}) \delta_{m}^{l} \delta\left(\mathbf{k}+\mathbf{k}^{\prime}\right) \delta\left(\omega+\omega^{\prime}\right) \\
\left\langle\hat{v}_{\alpha}^{l}(\mathbf{k}, \omega) \hat{v}_{\beta, m}\left(\mathbf{k}^{\prime}, \omega^{\prime}\right)\right\rangle & =\hat{G}(\mathbf{k}, \omega) \hat{\tau}_{\alpha \beta}(\mathbf{k}) \delta_{m}^{l} \delta\left(\mathbf{k}+\mathbf{k}^{\prime}\right) \delta\left(\omega+\omega^{\prime}\right)
\end{aligned}
$$

with $\hat{G}_{0}$ and $\hat{U}_{0}$ given by $(2.27)$ and (2.28).

Perturbation theory in $\lambda_{0} \mathcal{L}_{1}^{(N)}$ is also straightforward. The only changes are that one associates an extra index, $l$, with each line in a diagram, and the vertices are now $\frac{i}{2} \lambda_{0} P_{\alpha \beta \gamma}(\mathbf{k}) A_{N}^{l m n}$ and $\frac{i}{2} \lambda_{0} P_{\alpha \beta \gamma}(\mathbf{k}) A_{N, l m n}$, where $A_{N, l m n} \equiv A_{N}^{l m n *}$. We adopt the convention that an arrow coming into a vertex carries an upper index, while an arrow going out of a vertex carries a lower index (see Figure 2-2). 


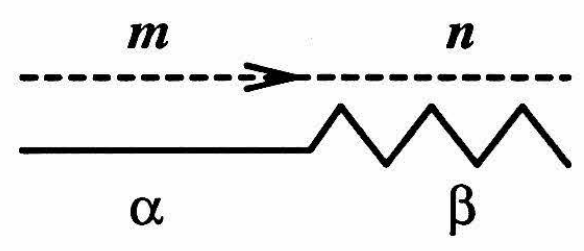

(a)

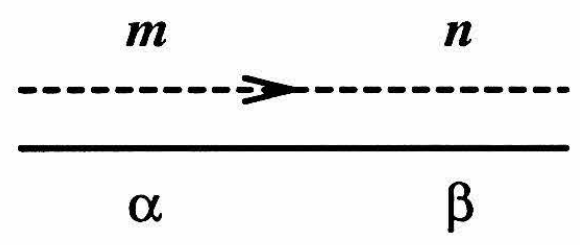

(b)

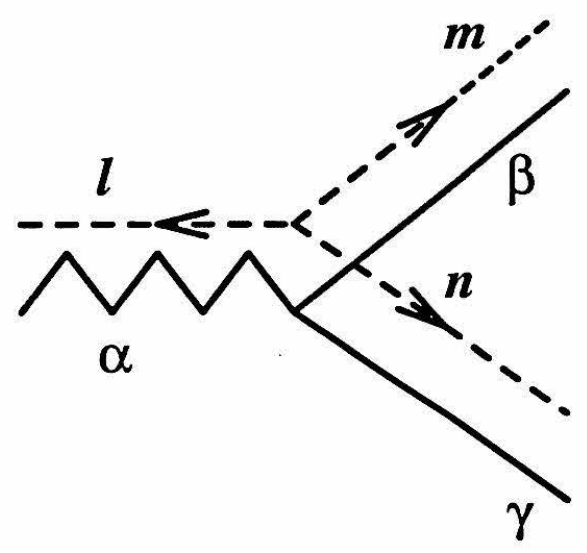

(c)

Figure 2-2: $(a) \hat{G}_{0} \hat{\tau}_{\alpha \beta} \delta_{m}^{n}$ (b) $\hat{U}_{0} \hat{\tau}_{\alpha \beta} \delta_{m}^{n}$ (c) $\frac{i}{2} \lambda_{0} P_{\alpha \beta \gamma} A_{l m n}^{N}$ 


\subsection{Symmetries of $\stackrel{\leftrightarrow}{A}_{N}$}

In order to limit our search for appropriate cubic invariants, we now discuss symmetry requirements on the coefficients $A_{N}^{l m n}$.

Firstly, since the projection-type operator $P_{\alpha \beta \gamma}(\mathbf{k})$ is symmetric under interchange of $\beta$ and $\gamma$, it is natural to choose $A_{N}^{l m n}$ symmetric in $m$ and $n$. It is easy to check that the equations of motion are still nontrivial even if $A_{N}^{l m n}$ is antisymmetric in $m$ and $n$ (the nonlinear term does not vanish identically so long as $N \geq 1$ ), but this does not give a sensible $N=1$ limit. In particular the diagonal terms, $n=m$, which are the only ones that survive when $N=1$, are cancelled. We therefore assume $A_{N}^{l m n}=A_{N}^{l n m}$.

Secondly, we impose the constraint that the total energy be conserved in the absence of viscosity and forcing. The total energy is defined as

$$
E=\int d^{d} r \epsilon(\mathbf{r}, t), \quad \epsilon(\mathbf{r}, t) \equiv \frac{1}{2} \rho_{0} \sum_{l=1}^{N} \mathbf{v}^{l}(\mathbf{r}, t) \cdot \mathbf{v}_{l}(\mathbf{r}, t)
$$

whose integrand reduces to $\frac{1}{2} \rho_{0} \sum_{l}\left|\mathbf{v}^{l}\right|^{2}$ when the representation is unitary, but is in any case real and positive. Using the incompressibility conditions, and appropriate integrations by parts, one finds

$$
\frac{d E}{d t}=-\frac{1}{2} \rho_{0} \lambda_{0} \operatorname{Re}\left\{\sum_{l, m, n}\left(A_{N}^{l m n}-A_{N}^{n m l}\right) \int d^{d} r \mathbf{v}_{l} \cdot\left(\mathbf{v}_{\mathbf{m}} \cdot \nabla\right) \mathbf{v}_{\mathbf{n}}\right\}
$$

and therefore vanishes automatically if $A_{N}^{l m n}=A_{N}^{n m l}$ (more complicated assumptions 
which allow for an antisymmetric part may be possible, but we again appeal to a sensible $N=1$ limit). Associated with this conservation law is the conserved energy current $\mathbf{j}_{E}(\mathbf{r}, t)=\operatorname{Re}\left\{\lambda_{0} \rho_{0} \sum_{l, m, n} A_{N}^{l m n}\left(\mathbf{v}_{l} \cdot \mathbf{v}_{n}\right) \mathbf{v}_{m}+2 \sum_{l} p^{l} \mathbf{v}_{l}\right\}$ such that $\frac{\partial \epsilon}{\partial t}+\nabla \cdot \mathbf{j}_{E}=0$. Together with the symmetry in $m$ and $n$, this implies that we require full symmetry under all permutations of the three indices:

$$
A_{N}^{l m n}=A_{N}^{l n m}=A_{N}^{n m l}=A_{N}^{m l n} .
$$

\subsection{Group Theoretical Considerations}

We now treat more technical issues involving the relation between $\overleftrightarrow{A}_{N}$ and the group $G$. We will discuss two approaches to constructing group invariants: trace invariants and Wigner symbols. The first is actually a special case of the second, but is easier to motivate, and hence worth introducing separately.

\subsubsection{Trace Invariants}

Perhaps the simplest way to generate invariants is to associate the indices $l, m$, and $n$ with the generators of the group $G$. Thus we let $\mathbf{J}^{l}, l=1, \ldots, N$ be some set of finite-dimensional Hermitean matrices such as the unitary matrix, $\mathbf{U}(g)$, representing 
any group element, $g \in G$, may be expressed as

$$
\mathbf{U}(g)=e^{i \sum_{l=1}^{N} a_{l}(g) \mathbf{J}^{\prime}}, \quad g \in G,
$$

where the $a_{l}$ are real numbers. The group structure is completely specified by the structure constants, $f^{l m n}$, which are real and defined by the commutation relations

$$
\left[\mathbf{J}^{l}, \mathbf{J}^{m}\right]=i \sum_{n=1}^{N} f^{l m n} \mathbf{J}^{n}
$$

We will always take $\operatorname{tr}\left[\mathbf{J}^{l}\right]=0$ (since an overall phase factor in (2.36) has no effect). By choosing suitable linear combinations if necessary, we may also take $\operatorname{tr}\left[\mathbf{J}^{l} \mathbf{J}^{m}\right]=$ $\lambda \delta_{l m}$, where the real number $\lambda>0$ is chosen for convenience. In this case $f^{l m n}=$ $\frac{1}{i \lambda} \operatorname{tr}\left\{\left[\mathbf{J}^{l}, \mathbf{J}^{m}\right] \mathbf{J}^{n}\right\}$ is completely antisymmetric in all three indices. For example, if $G=S U(2)$, and $\mathbf{J}^{l}=\sigma^{l}, l=1,2,3$, are the Pauli matrices, then $\lambda=2$, and $f^{l m n}=2 \epsilon_{l m n}$ where $\epsilon_{l m n}$ is the fully antisymmetric tensor with $\epsilon_{123}=1$.

Suppose we now define matrix dynamical variables, $\Omega_{\alpha}[\mathbf{x}]$, by

$$
\Omega_{\alpha}[\mathbf{x}]=\sum_{l=1}^{N} x_{\alpha, l} \mathbf{J}^{l}, \quad \alpha=1, \ldots, d
$$

where $\mathbf{x}^{l}(\mathbf{r}, t)=\mathbf{x}_{l}(\mathbf{r}, t)$ are real vector field dynamical variables. The unitary trans- 
formation (2.36) induces a transformation on the $\mathbf{x}^{l}$ via

$$
\Omega_{\alpha}\left[\mathbf{x}^{\prime}\right] \equiv \mathbf{U}^{\dagger}(g) \Omega_{\alpha}[\mathbf{x}] \mathbf{U}(g)=\sum_{l=1}^{N} x_{\alpha, l}^{\prime} \mathbf{J}^{l}
$$

which defines a $N \times N$ real orthogonal matrix $D_{N}^{l l^{\prime}}(g)$ via

$$
\begin{gathered}
\mathbf{U}^{\dagger}(g) \mathbf{J}^{l} \mathbf{U}(g)=\sum_{l^{\prime}=1}^{N} D_{N}^{l l^{\prime}}(g) \mathbf{J}^{l^{\prime}}, \\
x_{\alpha}^{\prime l}=\sum_{l^{\prime}=1}^{N} D_{N}^{l l^{\prime}}(g) x_{\alpha}^{l^{\prime}} ; \stackrel{\leftrightarrow}{D}_{N}^{T}(g) \overleftrightarrow{D}_{N}(g)=I_{N}
\end{gathered}
$$

[compare (2.6)] and there is no distinction between upper and lower indices in this case. We now ask: what equations of motion for the $\mathbf{x}_{\alpha}^{l}$ can we write down that are invariant under this group of transformations? To see the answer, note that due to the cyclic property of the trace, any quantity of the form

$$
\operatorname{tr}\left\{\Omega_{\alpha}[\mathbf{x}] \Omega_{\beta}[\mathbf{y}] \ldots \Omega_{\gamma}[\mathbf{z}]\right\} \equiv \sum_{l_{1}, l_{2}, \ldots, l_{m}} A_{N}^{l_{1} \ldots l_{m}} x_{\alpha, l_{1}} y_{\beta, l_{2}} \ldots z_{\gamma, l_{m}}
$$

where,

$$
A_{N}^{l_{1} \ldots l_{m}} \equiv \operatorname{tr}\left\{\mathbf{J}^{l_{1}} \ldots \mathbf{J}^{l_{m}}\right\}
$$

is an invariant of order $m$. Invariants other than those defined by (2.43) can also be constructed: simply contract the indices on products of lower order $\overleftrightarrow{A}_{N}$ 's. For example, both $\delta_{l_{1} l_{2}} \delta_{l_{3} l_{4}}$ and $\sum_{n} A_{N}^{l_{1} l_{2} n} A_{N}^{n l_{3} l_{4}}$ are fourth order invariants (which may or 
may not differ significantly from $\left.A_{N}^{l_{1} l_{2} l_{3} l_{4}}\right)$.

Consider then any set of equations of motion of the form

$$
\dot{x}_{\alpha}^{l}=\mathcal{F}_{\alpha}^{l}[\mathbf{x}], l=1, \ldots, N, \alpha=1, \ldots, d,
$$

where $\mathcal{F}_{\alpha}^{l}[\mathbf{x}]$ is a sum over all possible $m$, and for each $m$, all possible $m$ th order invariants (which we will still denote generically by $A_{N}^{l_{1} \ldots l_{m}}$ ), of terms of the form

$$
\mathcal{F}_{\alpha}^{l ; m}[\mathbf{x}] \equiv \sum_{l_{2}, \ldots, l_{m}} A_{N}^{l l_{2} \ldots l_{m}} \sum_{\alpha_{2}, \ldots, \alpha_{m}} O_{\alpha \alpha_{2} \ldots \alpha_{m}} x_{\alpha_{2}, l_{2}} \ldots x_{\alpha_{m}, l_{m}}
$$

where $O_{\alpha \alpha_{2} \ldots \alpha_{m}}$ could be any spatial-rotation invariant integro-differential operator acting of the $\mathbf{r}$ dependences of the $x_{\alpha}^{l}(\mathbf{r}, t)$. In particular, the form

$$
O_{\alpha \alpha_{2} \alpha_{3}}=\delta_{\alpha \alpha_{3}} \int d^{d} r_{2} \int d^{d} r_{3} \delta\left(\mathbf{r}-\mathbf{r}_{2}\right) \frac{\partial}{\partial r_{\alpha_{2}}} \delta\left(\mathbf{r}-\mathbf{r}_{3}\right)
$$

yields the nonlinear term in (2.1) with $\mathbf{x}^{l} \equiv \mathbf{v}^{l}$. These equations must transform covariantly since multiplying by $x_{\alpha, l}$ and summing on $l$ yields a scalar on both sides of the equation.

Given a group $G$, there are many possible choices for the generators $\mathbf{J}^{l}$; however, within a given representation, any choice may be obtained from any other by taking appropriate linear combinations. The corresponding invariants are then similar linear combinations of each other. The number, $N$, of generators is determined by the 
dimension of the group. Thus, if $N$ is to vary (in particular, become large) the group $G$ must vary with $N$. The dimension of the matrices $\mathbf{J}^{l}$ (i.e., the dimension of the representation) is of no consequence here. Though the details of the representation may enter [more than just the structure constants (2.37) are relevant in computing (2.43)], the matrices $\overleftrightarrow{D}_{N}$ are representation independent, and a single maximal set of independent invariants must exist. It is simplest to assume that the $\mathbf{J}^{l}$ generate the fundamental representation, i.e., they have minimal dimension. For $S U(2)$ these are the Pauli matrices; for $S U(n)$ they are any orthogonal set of $N=n^{2}-1, n \times n$ traceless matrices. Below we will discuss invariants that depend more significantly on the representation.

In the example of $S U(2)$ represented by the Pauli matrices, the first few invariants are

$$
\begin{aligned}
A_{3}^{l} & =\operatorname{tr}\left(\sigma^{l}\right)=0 \\
A_{3}^{l_{1} l_{2}} & =\operatorname{tr}\left(\sigma^{l_{1}} \sigma^{l_{2}}\right)=2 \delta_{l_{1} l_{2}} \\
A_{3}^{l_{1} l_{2} l_{3}} & =\operatorname{tr}\left(\sigma^{l_{1}} \sigma^{l_{2}} \sigma^{l_{3}}\right)=2 i \epsilon_{l_{1} l_{2} l_{3}} \\
A_{3}^{l_{1} l_{2} l_{3} l_{4}} & =\operatorname{tr}\left(\sigma^{l_{1}} \sigma^{l_{2}} \sigma^{l_{3}} \sigma^{l_{4}}\right)=2\left[-\delta_{l_{1} l_{3}} \delta_{l_{2} l_{4}}+\delta_{l_{1} l_{4}} \delta_{l_{2} l_{3}}+\delta_{l_{1} l_{2}} \delta_{l_{3} l_{4}}\right] .
\end{aligned}
$$

Note that $A^{l m n}=i f^{l m n}$ is completely antisymmetric in this case, and therefore violates the requirements in Section 2.3. It will always be the case that $A_{N}^{l m n}-A_{N}^{m l n}=$ $i \lambda f^{l m n}$. The only question is whether or not there is a nontrivial symmetric part 
[clearly not, for $S U(2)]$. Note also that the fourth order invariant can be constructed trivially from sums of products of the second order one. In fact, in this case all higher order invariants may be constructed from products of Kronecker- $\delta$ 's and $\epsilon$-tensors. This implies the well-known result that all rotation invariant combinations of vectors in three dimensions may be constructed from dot products and cross products.

\subsubsection{Wigner Coefficients}

In the second approach to constructing invariants we associate the index $l$ with the basis vectors (or states) on which the $\mathbf{J}^{l}$ operate. Thus $N$ is now the dimension of the matrices rather than their number, and may vary even when the group $G$ is fixed, independent of $N$. Using a quantum mechanical bra-ket notation, if $\{|1\rangle, \ldots,|N\rangle\}$ is an orthonormal basis for the vector space (with corresponding hermitian conjugates $\{\langle 1| \ldots,\langle N|\})$, then we define dynamical states

$$
\left|x_{\alpha}\right\rangle=\sum_{l=1}^{N} x_{\alpha}^{l}|l\rangle,\left\langle x_{\alpha}\right|=\sum_{l=1}^{N} x_{\alpha, l}\langle l|, l=1, \ldots, d
$$

The operation

$$
\left|x_{\alpha}^{\prime}\right\rangle=\mathbf{U}(g)\left|x_{\alpha}\right\rangle,\left\langle x_{\alpha}^{\prime}\right|=\left\langle x_{\alpha}\right| \mathbf{U}^{\dagger}(g)
$$

then defines the group of transformations on the $x_{\alpha}^{l}$ via

$$
x_{\alpha}^{\prime l}=\sum_{l^{\prime}=1}^{N} D_{N}^{l l^{\prime}}(g) x_{\alpha}^{l^{\prime}}, x_{\alpha, l}^{\prime}=\sum_{l^{\prime}=1}^{N} D_{N}^{l l^{\prime}}(g)^{*} x_{\alpha, l^{\prime}}
$$


where

$$
D_{N}^{l l^{\prime}}(g)=\left\langle l|\mathbf{U}(g)| l^{\prime}\right\rangle
$$

For the group $S U(2)$, the representations are labelled by the total spin, $j=$ $0, \frac{1}{2}, 1, \frac{3}{2}, 2, \ldots$, and have dimension $N=2 j+1$. A convenient basis is formed by the eigenstates of angular momentum component $J_{z}$ :

$$
|l\rangle \equiv|j, m\rangle, \quad-j \leq m=l-j-1 \leq j .
$$

For integer $j$ these are most familiar in the form of the spherical harmonics $Y_{j m}(\theta, \phi)$. The transformation matrices (2.50) are the famous quantum mechanical $D$-matrices[22].

We now wish to construct quantities of the form

$$
\mathcal{I}_{\alpha_{1} \ldots \alpha_{m}}=\sum_{l_{1}, \ldots, l_{m}} A_{N}^{l_{1} \ldots l_{m}} x_{\alpha_{1}, l_{1}} \ldots x_{\alpha_{m}, l_{m}}
$$

where $\overleftrightarrow{A}_{N}$ is chosen to make $\mathcal{I}$ invariant under (2.50). Since, by (2.51),

$$
\mathbf{U}(g)|l\rangle=\sum_{l^{\prime}=1}^{N} D_{N}^{l^{\prime} l}(g)\left|l^{\prime}\right\rangle
$$

the kets transform in the same way that the $x_{\alpha, l}$ do. We may therefore state the problem alternatively: we seek a tensor, $\overleftrightarrow{A}_{N}$, such that the linear combination of 
product states

$$
\left|\mathcal{I}^{(m)}\right\rangle=\sum_{l_{1}, \ldots, l_{m}} A_{N}^{l_{1} \ldots l_{m}}\left|l_{1}\right\rangle \otimes \ldots \otimes\left|l_{m}\right\rangle
$$

is a scalar (normalized in some fashion) under the transformation (2.54). For the group $S U(2)$ this means that we wish to add $m$ angular momenta together to obtain a state with zero total angular momentum. This is a well known problem in group theory and quantum mechanics, closely related to the problem of decomposing the direct product of $(m-1)$ irreducible representations of a group into a direct sum of irreducible representations. The solutions $A_{N}^{l_{1} \ldots l_{m}}$ are called Wigner coefficients. For $S U(2)$, with basis states given by (2.52), one uses the notation

$$
A_{N}^{l_{1} l_{2} l_{3}} \equiv\left(\begin{array}{ccc}
j & j & j \\
& & \\
m_{1} & m_{2} & m_{3}
\end{array}\right), \quad m_{i}=-j,-j+1, \ldots, j
$$

These are special cases of the Wigner $3 j$-symbols[22]. In general, the states in the direct product (2.54) can belong to different representations, $N_{1}, \ldots, N_{m}$ and we would seek coefficients $A_{N_{1} \ldots N_{m}}^{l_{1} \ldots l_{m}}$ that make the result a scalar. The general $S U(2)$ Wigner $3 j$-symbol is then $\left(\begin{array}{ccc}j_{1} & j_{2} & j_{3} \\ & & \\ m_{1} & m_{2} & m_{3}\end{array}\right) \equiv A_{N_{1} N_{2} N_{3}}^{l_{1} l_{2} l_{3}}$, with $N_{i}=2 j_{i}+1$ and $m_{i}=l_{i}-j_{i}-1$. We are clearly interested only in the case in which all representations are the same.

Appropriate equations of motion may now be written down precisely as before. Equations (2.45) and (2.46) are valid with the new tensors $\overleftrightarrow{A}_{N}$ defined above.

In addition, the relation to the Clebsch-Gordon coefficients is made clear by con- 
structing the state

$$
\left|\mathcal{I}_{l}^{(m)}\right\rangle=\sum_{l_{2}, \ldots, l_{m}} A_{N}^{l l_{2} \ldots l_{m}}\left|l_{2}\right\rangle \otimes\left|l_{3}\right\rangle \otimes \ldots \otimes\left|l_{m}\right\rangle
$$

From (2.55) we have the obvious relation

$$
\left|\mathcal{I}^{(m)}\right\rangle=\sum_{l=1}^{N}|l\rangle \otimes\left|\mathcal{I}_{l}^{(m)}\right\rangle
$$

Since $\left|\mathcal{I}^{(m)}\right\rangle$ is a scalar, it is clear that $\left|\mathcal{I}_{l}^{(m)}\right\rangle$ must transform in the same way that $\langle l|$ does, i.e., with the complex conjugate representation. More generally, if the states in the product come from different representations, $N_{2}, \ldots, N_{m}$, the resulting states transform via the complex conjugate of the representation $N$. Thus by letting $N$ vary over all permitted values, the Wigner symbols allow one to decompose the transformation of the given direct product into a direct sum of irreducible representations (this is known as the Clebsch-Gordon decomposition of the direct product). This does not quite define the usual Clebsch-Gordon coefficients. These are defined so that the resulting product state transforms via the representation $N$, not its complex conjugate. In the case where the representation is real (i.e., where the complex conjugate representation is the same as the representation itself), there is a matrix $g^{l l^{\prime}}$ such that

$$
|l\rangle=\sum_{m=1}^{N} g^{l l^{\prime}}\left\langle l^{\prime}\right|
$$


translates the bras into kets. This matrix is really a special case of the invariant tensors $\overleftrightarrow{A}_{N}$ in which there are only two indices since $\left|\mathcal{I}^{(2)}\right\rangle=\sum_{l, l^{\prime}} g^{l l^{\prime}}|l\rangle \otimes\left|l^{\prime}\right\rangle$ is clearly a scalar. If we then define the Clebsch-Gordon coefficients

$$
C_{N ; N_{2} \ldots N_{m}}^{l ; l_{2} \ldots l_{m}}=c\left(N, N_{1}, \ldots, N_{m}\right) \sum_{l^{\prime}=1}^{N} g^{l l^{\prime}} A_{N N_{2} \ldots N_{m}}^{l^{\prime} l_{2} \ldots l_{m}}
$$

where the prefactor is an appropriate normalization, it is clear that

$$
\left|\overline{\mathcal{I}}_{l}^{(m)}\right\rangle=\sum_{l_{2}, \ldots, l_{m}} C_{N ; N_{2} \ldots N_{m}}^{l_{j} l_{1} \ldots l_{m}}\left|l_{2}\right\rangle \otimes \ldots \otimes\left|l_{m}\right\rangle
$$

transforms just as $|l\rangle$ does. For $m \geq 4$ these coefficients are no more unique than the Wigner coefficients are [see the discussion below (2.43)]. For the rotation group $O(3)$ the representations are all real, and one more commonly denotes

$$
C_{N ; N_{2} N_{3}}^{l ; l_{2} l_{3}} \equiv\left\langle j m \mid j_{2} m_{2} j_{3} m_{3}\right\rangle=(-1)^{j_{2}-j_{3}+m} \sqrt{2 j+1}\left(\begin{array}{ccc}
j & j_{2} & j_{3} \\
-m & m_{2} & m_{3}
\end{array}\right),
$$

corresponding to the matrix $g^{l l^{\prime}}=(-1)^{l^{\prime}} \delta_{l^{\prime}, N-l}$, and conventional normalization $c\left(N, N_{1}, N_{2}\right)=-(-1)^{\frac{1}{2}\left(N_{2}-N_{3}\right)-N} \sqrt{N}[22]$.

Finally, we mentioned at the beginning of this section that the trace invariants are really special cases of the Wigner coefficients. They are constructed, in effect, by using the group generators as states. This, in fact, corresponds to a special representation, known as the adjoint representation[23], and it can be shown that in this 
representation the Wigner coefficients as defined above are precisely the traces of products of generators, (2.43). The demonstration of this fact is a special case of the use of tensor methods in the theory of group representations[23], where invariants are constructed as traces of products of more general tensor quantities. In the case of the adjoint representation, the tensors have only two indices [one transforming under the fundamental representation, the other under its complex conjugate, exactly as in (2.39)], and are traceless, and hence may always be written as linear combinations of the generators, precisely as in (2.38).

\subsection{Graphical Considerations}

A crucial property of the group-theoretical formulation is the preservation of the coefficients $A_{N}^{l_{1} \ldots l_{m}}$ under vertex renormalization. Stated more simply, different order diagrams, with the same structure of $m$ external legs, will have the same dependence on the indices $l_{1}, \ldots, l_{m}$, and must therefore be proportional to some linear combination of $\overleftrightarrow{A}_{N}$ 's with the same $m$. For small $m$ ( $m=2$ or 3 , say) there will be only a single type of $\overleftrightarrow{A}_{N}$, and the dependence on the indices $l_{1}, \ldots, l_{m}$ will be uniquely specified.

The reason for this is that $\mathcal{L}_{1}^{(N)}$ is a scalar, and therefore the average of an operator $\mathcal{O}^{l_{1}, \ldots, l_{m}}$, which transforms in the same way that $x^{l_{1}} y^{l_{2}} \ldots z^{l_{m}}$ does, is given by the sum over $p$ of

$$
O_{p}^{l_{1} \ldots l_{m}} \equiv \lambda_{0}^{p}\left\langle\mathcal{O}^{l_{1} \ldots l_{m}}\left(\mathcal{L}_{1}^{(N)}\right)^{p}\right\rangle_{0}
$$


which therefore must also transform in the same way. Now $O_{p}^{l_{1} \ldots l_{m}}$ is independent of the $\mathbf{v}$ and $\mathbf{w}$ fields, and a contraction of the form

$$
O_{p}=\sum_{l_{1} \ldots l_{m}} O_{p}^{l_{1} \ldots l_{m}} x_{l_{1}} y_{l_{2}} \ldots z_{l_{m}}
$$

is, by construction, a scalar. However, the $A_{N}^{l_{1} \ldots l_{m}}$ comprise all invariants; therefore, $O_{p}^{l_{1} \ldots l_{m}}$ must be some linear combination of them.

At the level of graphical technology, it is precisely this property that is responsible for the variation of the universality class with $N$ in the spin models. Without the group symmetry, diagrams with the same external leg structure would have essentially random dependence on the indices and will therefore not add up in any coherent way. This is precisely what leads to Ising-like behavior for all finite $N$. We expect similar behavior to occur in the turbulence problem.

\subsection{Galilean Invariance}

In Chapter 1 we alluded to the importance of Galilean invariance in the establishment of the exact renormalization group results (1.15) and (1.18) for the exponents. If the generalization to $N>1$ is to be truly useful, it seems necessary that we be able to prove similar results for any $N$. This can, in fact, be done, and is a consequence only of the symmetries, (2.35). 
For $N=1$ the Galilean transformation

$$
\mathbf{v}^{\prime}(\mathbf{r}, t)=\mathbf{v}\left(\mathbf{r}+\lambda_{0} \mathbf{v}_{0} t, t\right)-\mathbf{v}_{0}
$$

where $\mathbf{v}_{0}$ is an arbitrary fixed (real) velocity, leaves the Navier-Stokes equations invariant since

$$
\frac{\partial \mathbf{v}^{\prime}}{\partial t}+\lambda_{0}\left(\mathbf{v}^{\prime} \cdot \nabla\right) \mathbf{v}^{\prime}=\frac{\partial \mathbf{v}}{\partial t}+\lambda_{0}(\mathbf{v} \cdot \nabla) \mathbf{v}
$$

If, in addition, we assume that $\mathbf{w}^{\prime}(\mathbf{r}, t)=\mathbf{w}\left(\mathbf{r}+\lambda_{0} \mathbf{v}_{0} t, t\right)$ transforms without an additive term (as do $\mathbf{f}$ and $p$ ), then the Lagrangian (2.20) is Galilean invariant, $\mathcal{L}\left[\mathbf{v}^{\prime}, \mathbf{w}^{\prime}\right]=\mathcal{L}[\mathbf{v}, \mathbf{w}]$. It is precisely this invariance that was exploited by DeDominicis and Martin[4b] to prove (1.15) and (1.18) to all orders in $y$.

For $N>1$, consider the following generalization of (2.65):

$$
\mathbf{v}^{\prime l}(\mathbf{r}, t)=\mathbf{v}^{l}\left(\mathbf{r}+\lambda_{0} \mathbf{v}_{0} t, t\right)-\frac{1}{\mu} h^{l} \mathbf{v}_{0} ; \mathbf{v}_{l}^{\prime}(\mathbf{r}, t)=\mathbf{v}_{l}\left(\mathbf{r}+\lambda_{0} \mathbf{v}_{0} t, t\right)-\frac{1}{\mu^{*}} h_{l} \mathbf{v}_{0},
$$

where $h^{l}=h_{l}^{*}$ are any set of complex numbers, normalized so that $\sum_{l=1}^{N} p_{l} p^{l}=1$, and $\mu$ is yet to be determined. We then have

$$
\begin{aligned}
\frac{\partial \mathbf{v}^{\prime l}}{\partial t}+\lambda_{0} \sum_{m, n} A_{N}^{l m n}\left(\mathbf{v}_{m}^{\prime} \cdot \nabla\right) \mathbf{v}_{n}^{\prime} & =\frac{\partial \mathbf{v}^{l}}{\partial t}+\lambda_{0} \sum_{m, n} A_{N}^{l m n}\left(\mathbf{v}_{m} \cdot \nabla\right) \mathbf{v}_{n} \\
& +\lambda_{0}\left(\mathbf{v}_{0} \cdot \nabla\right)\left[\mathbf{v}^{l}-\frac{1}{\mu} \sum_{n} A_{N}^{l n}(h) \mathbf{v}_{n}\right]
\end{aligned}
$$

where $A_{N}^{l n}(h)=\sum_{m} A_{N}^{l m n} h_{m}$. The last term on the right cannot generally be made 
to vanish simultaneously for all $l$, so full invariance of the equations of motion under (2.67) is not possible. Let us focus instead on the equation of motion for scalar combinations

$$
\mathbf{v}_{q}(\mathbf{r}, t)=\frac{1}{2} \sum_{l=1}^{N}\left[q_{l} \mathbf{v}^{l}(\mathbf{r}, t)+q^{l} \mathbf{v}_{l}(\mathbf{r}, t)\right]
$$

where $q^{l}=q_{l}^{*}$ is another set of complex numbers with $\sum_{l} q^{l} q_{l}=1$. From (2.68), the equation of motion for $\mathbf{v}_{q}$ is invariant under the transformation (2.67) only if

$$
\mu q^{n}=\sum_{l=1}^{N} A_{N}^{l n}(h) q_{l}=\sum_{l, m} A_{N}^{l m n} h_{m} q_{l} .
$$

This is a kind of eigenvalue problem for the $q^{n}$, with eigenvalue $\mu$. The matrix $A_{N}^{l n}(h)$ is symmetric, but not necessarily real, so $\mu$ is not necessarily real.

Associated, then, with each Galilean transformation, (2.67), are a set of $N$ invariant velocity fields, $\mathbf{v}_{q}(\mathbf{r}, t)$, one for each eigenvector $q^{l}$ of $A_{N}^{l n}(h)$, transforming with the associated eigenvalue, $\mu$. This invariance property clearly respects the group symmetry: if $\mathbf{v}^{l}$ is transformed according to (2.6), transforming $h^{l}$ and $q^{l}$ in exactly the same way yields the same scalar $\mathbf{v}_{q}$ in (2.69), and the same eigenvalue equation (2.70). Clearly, when $N=1$ the standard Galilean invariance, (2.65) and (2.66), is recovered with eigenvalue $\mu=1$.

These invariance properties, though compelling, do not by themselves imply the result we seek, namely that the renormalization group results for the exponents generalize to any $N>1$. Further analysis is necessary: in Appendix A we present the 
technical arguments necessary to fully establish (1.15) and (1.18). The validity of these results lends further credence to our proposition that the $N>1$ equations really do represent a logical generalization of the $N=1$ Navier-Stokes equations, and do not violate any fundamental symmetries present at $N=1$. One might still worry that the Galilean symmetry at $N=1$ is somehow stronger and more profound than the seemingly more restrictive symmetry inherent in (2.67) and (2.70). However we know of no explicit property (of the correlation functions, for example) that demonstrate any "discontinuity" in behavior between $N=1$ and $N>1$. Unless such behavior is found, it seems reasonable to propose that the model evolves continuously with $N$, just as do the $N$-vector models of magnetism. 


\section{Chapter 3}

\section{The Spherical Limit $(N \rightarrow \infty)$}

\subsection{Graph Decomposition Rules}

Before deriving the spherical limit, we shall first develop some useful graph decomposition rules in this section. These rules concern the numerical factors due to the presence of $A_{N}^{l m n}$ and thus have nothing to do with the spatial parts of diagrams. Their validity is very general and can be applied to a very general class of $A_{N}^{l m n}$ or even to other theories, such as theories with a quartic term ( $\phi^{4}$ theory).

These rules are summarized in Figure 3-1[24]. To derive them, we first make a few remarks about the group transformation properties of perturbation theory developed from (2.29) and (2.30). First, as an example, let us consider the $\mathrm{n}^{\text {th }}$ order term of the two-point correlation function $\left\langle i \mathbf{w}^{l} \cdot \mathbf{v}_{l^{\prime}}\right\rangle$, which may be written as

$$
\left\langle i \mathbf{w}^{l} \cdot \mathbf{v}_{l^{\prime}}\left[\lambda_{0} \mathcal{L}_{1}^{(N)}\right]^{n}\right\rangle_{0}
$$

where the average $\langle\cdot\rangle_{0}$ is done with respect to $\mathcal{L}_{0}^{(N)}$. Under the group transformation, 


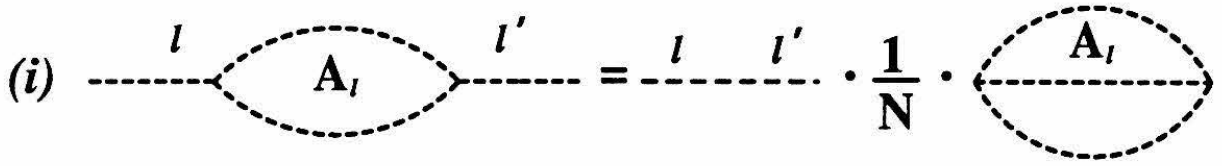

(ii)

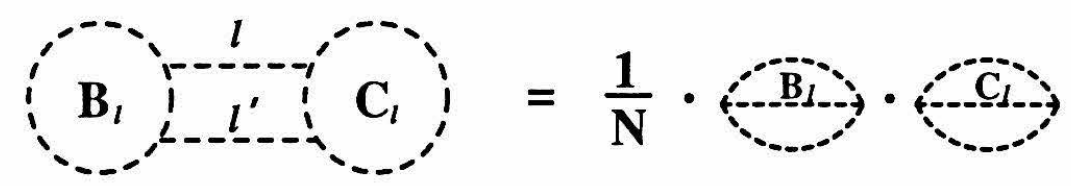

(iii)<smiles></smiles><smiles>CC(C)I=[IH]</smiles>

(iv)
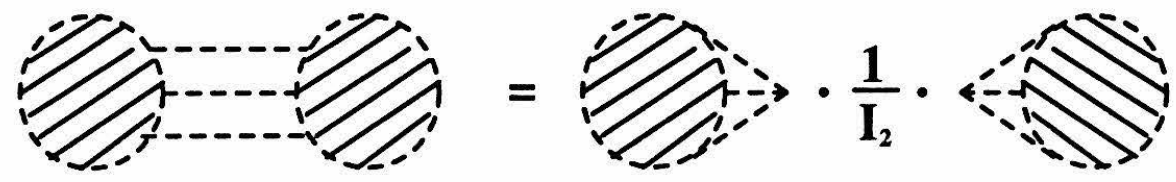

Figure 3-1: Graph decomposition rules 
the term (3.1) transforms exactly as $\mathbf{w}^{l} \cdot \mathbf{v}_{l^{\prime}}$ because $\mathcal{L}_{0}^{(N)}$ and $\mathcal{L}_{1}^{(N)}$ are invariant. Now assuming $\mathbf{w}^{l}$ and $\mathbf{v}_{l^{\prime}}$ transform according to the irreducible representations of the group $G$, then by the fundamental lemma, Schur's lemma, of group representation theory, it is straightforward to show that $\delta_{l}^{\prime \prime}$ is the only invariant second rank tensor under the group transformation. Hence (3.1) must be proportional to $\delta_{l}^{l^{\prime}}$ with an $l$-independent proportional constant (see Section 2.5). Similarly, assuming $A_{N}^{l m n}$ is the only invariant three rank tensor, any three-point correlation function is also proportional to $A_{N}^{l m n}$.

Under these assumptions, rule $(i)$ is simply the graphic representation of the following identity:

$$
\text { the left hand side }=\delta_{l}^{l^{\prime}} \cdot A_{l}=\delta_{l}^{\prime \prime} \cdot \frac{1}{N} \cdot \sum_{l} A_{l}
$$

where $\sum_{l} A_{l}$ corresponds to the closed diagram on the right hand side.

By using rule $(i)$, rule $(i i)$ can be easily derived as follows:

the left hand side $=\sum_{l, l^{\prime}} B_{l} C_{l} \delta_{l^{\prime}}^{l}=\sum_{l} B_{l} C_{l}=N B_{l} C_{l}=\frac{1}{N} \sum_{l_{1}} B_{l_{1}} \sum_{l_{2}} C_{l_{2}}$.

The right hand side of rule ( $i i)$ is thus the graphic representation of the last expression.

To derive rule (iii), we first note that since $\left\langle i \mathbf{w}^{l} \mathbf{v}^{m} \mathbf{v}^{n}\right\rangle$ is assumed to be proportional to $A_{N}^{l m n}$, it can be written as $A_{N}^{l m n} \Gamma_{N}$, where $\Gamma_{N}$ is the proportional constant. 
Therefore, we have

$$
\sum_{l m n} A_{l m n}^{N}\left\langle i \mathbf{w}^{l} \mathbf{v}^{m} \mathbf{v}^{n}\right\rangle=\Gamma_{N} \cdot \sum_{l m n} A_{l m n}^{N} A_{N}^{l m n}
$$

Hence $\Gamma_{N}=\frac{1}{I_{2}} \sum_{l m n} A_{l m n}^{N}\left\langle i \mathbf{w}^{l} \mathbf{v}^{m} \mathbf{v}^{n}\right\rangle$, where $I_{2}$ is $\sum_{l m n} A_{l m n}^{N} A_{N}^{l m n}$. Finally, we have

$$
\left\langle i \mathbf{w}^{l} \mathbf{v}^{m} \mathbf{v}^{n}\right\rangle=\sum_{l^{\prime} m^{\prime} n^{\prime}} A_{l^{\prime} m^{\prime} n^{\prime}}^{N}\left\langle i \mathbf{w}^{l^{\prime}} \mathbf{v}^{m^{\prime}} \mathbf{v}^{n^{\prime}}\right\rangle \cdot A_{N}^{l m n} \cdot \frac{1}{I_{2}}
$$

This is the content of the third rule. Using rule (ii), rule $(i v)$ can be derived similarly to rule $(i i)$.

\section{$3.2 \quad N \rightarrow \infty$}

As far as two-point correlation functions are concerned, only the first two rules are relevant. For any diagrams of two-point correlation functions, we first close them by using rule $(i)$. If the closed diagrams can be separated into two diagrams by severing two internal lines, they are so-called two-particle reducible (2PR) diagrams. They can be decomposed into two smaller diagrams by using rule (ii). These two smaller diagrams may be decomposed further if they are also two-particle reducible. This reduction can be proceeded until finally we are left with a bunch of two-particle irreducible (2PI) diagrams and some $\frac{1}{N}$ factors. Some of the lower order terms of $2 \mathrm{PI}$ diagrams are shown in Figure 3-2. If during this reduction, no other 2PI diagrams except the lowest order ones (the first term shown in Figure 3-2) are generated, they 


\section{7}

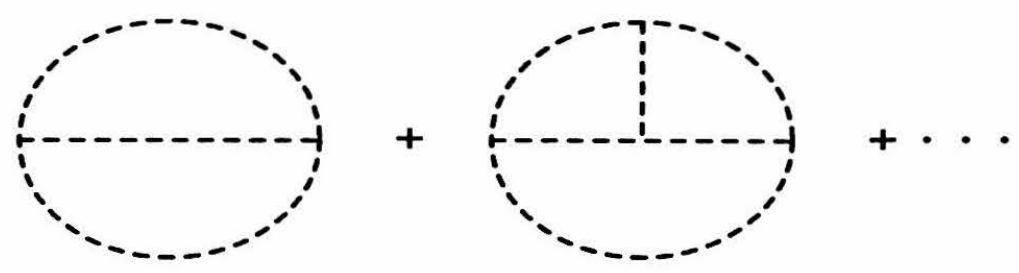

Figure 3-2: Lower order terms of two particle irreducible diagrams

are called fully $2 \mathrm{PR}$ diagrams.

In general, any $2 \mathrm{PI}$ diagram has an even number of vertices. Therefore, we may represent them by $I_{2}, I_{4}, \cdots, I_{2 k}^{(i)}, \cdots$. Here, the lower indices indicate number of vertices in diagrams, while the upper indices indicate different topologies of the diagrams, because there may exist more than one diagram for a given number of vertices. When there is only one diagram for the given number of vertices, we drop the upper indices. Note that $I_{2}$ is the same as the factor defined in the last section that results from the lowest order 2PI diagram.

The total number of $\frac{1}{N}$ factors depends on how many times we sever the diagram. Suppose that at the end of this reduction, the 2PI diagrams we are left with are $\left(I_{2}\right)^{n_{1}}\left(I_{4}\right)^{n_{2}} \cdots\left(I_{2 k}^{(i)}\right)^{n_{k}^{i}} \ldots$. In other words, we are left with $n_{1} I_{2^{-}}$diagrams, $n_{2} I_{4^{-}}$ diagrams, etc. The total number of times we sever the diagram is then simply $\left(n_{1}+\right.$ $\left.n_{2}+\cdots+n_{k}^{i} \cdots\right)-1$. Adding the $\frac{1}{N}$ that was generated when we closed the diagram by using rule $(i)$, we have

$$
\text { any diagram }=\delta_{l}^{l^{\prime}}\left(\frac{1}{N}\right)^{n_{1}+\cdots+n_{k}^{i} \cdots}\left(I_{2}\right)^{n_{1}}\left(I_{4}\right)^{n_{2}} \cdots\left(I_{2 k}^{(i)}\right)^{n_{k}^{i}} \cdots
$$


For the special case when the diagrams are fully $2 \mathrm{PR}, n_{k}^{i}=0$ for $k \geq 2$. Therefore, we have

$$
2 \mathrm{n}^{\text {th }} \text { order fully } 2 \mathrm{PR} \text { diagram }=\delta_{l}^{l^{\prime}}\left(\frac{I_{2}}{N}\right)^{n} .
$$

It is now clear that the large- $N$ limit of any diagram is summarized in the asymptotic behaviors of $I_{2}, I_{4}, \cdots$ when $N \rightarrow \infty$. To discuss these asymptotic behaviors, it is convenient to choose the normalization: $I_{2}=N$. Then, under this normalization, we parameterize the asymptotic behavior of $I_{2 k}^{(i)}$ by $I_{2 k}^{(i)}=O\left(N^{1-\alpha_{k}^{i}}\right)$. As a result, (3.2) becomes

$$
\text { any diagram }=\delta_{l}^{l^{\prime}} \frac{1}{N^{n_{1} \alpha_{1}+n_{2} \alpha_{2}+\cdots+n_{k}^{i} \alpha_{k}^{i} \cdots}}
$$

where by construction, $\alpha_{1}=0$. Clearly, if all $\alpha_{k}^{i}$ s are positive, all the higher terms $\left(n_{k}^{i} \neq 0, k \geq 2\right)$ vanish when $N \rightarrow \infty$. In this case, fully $2 \mathrm{PR}$ diagrams are the only surviving diagrams in spherical limit. On the other hand, if some $\alpha_{k}^{i}$ is negative, it means that the previous normalization $I_{2}=N$ is not good. Suppose the most negative term is $\alpha_{k_{0}}^{i_{0}}$. Then the new normalization is simply $I_{2 k_{0}}^{i_{0}}=N$. The corresponding $2 \mathrm{PI}$ diagrams are then the surviving diagrams.

Although the above procedure of finding the spherical limit is quite general, without considering specific $A_{N}^{l m n}$, we are not able to make general statements about what kinds of diagrams can survive. Therefore, in this thesis we shall only consider the Wigner $3 j$ coefficients of $\mathrm{SU}(2)$, which is the group that has been understood most. We leave other choices of $A_{N}^{l m n}$ as possible future generalizations of this work (see 
Appendix D for one of our attempts).

Specifically, the $A_{N}^{l m n}$ we consider are

$$
A_{l m n}^{N}=f(N)\left(\begin{array}{ccc}
j & j & j \\
l & m & n
\end{array}\right) ; \quad l, m, n=-j,-j+1, \ldots, j
$$

where $N=2 j+1 . f(N)$ is introduced for the purpose of normalization and will be chosen below. In order to satisfy the symmetry requirements (2.35), we require that $j$ is an even integer (if $j$ is odd, $3 j$ coefficients will pick up a minus when exchanging two columns).

As it was mentioned in Chapter 2, when $j$ is an integer, the upper indices (e.g., $\left.\mathbf{v}^{m}\right)$ transform in the same manner as $Y_{j m}(\theta, \phi)$ does. Because $(-1)^{m} Y_{j-m}(\theta, \phi)=$ $Y_{j m}^{*}(\theta, \phi)$ and, by construction, $\left(\mathbf{v}^{m}\right)^{*}=\mathbf{v}_{m}$, the lowering and raising operations of indices are defined via

$$
A_{m} \equiv(-1)^{m} A^{-m}, A^{m} \equiv(-1)^{m} A_{-m}
$$

Thus, $\sum_{m} A_{m} B^{m}=\sum_{m} A^{m} B_{m}$. Altogether, the $3 j$ coefficients we consider satisfy

$$
\left(\begin{array}{lll}
j & j & j \\
l & m & n
\end{array}\right)=\left(\begin{array}{ccc}
l & m & n \\
j & j & j
\end{array}\right)=\left(\begin{array}{ccc}
j & j & j \\
-l & -m & -n
\end{array}\right)=\text { any permutations in } l, m, n .
$$

Finding the large- $N$ limit can be proceeded as we just described. First, we note that $3 j$ coefficients are not zero only when $l+m+n=0$. Apparently, this implies 
the "conservation" of indices at each vertex, which in turn implies that (3.1) is proportional to $\delta_{l}^{\prime}$. Since for a given $j$ the representations of $\mathrm{SU}(2)$ are irreducible, the proportional constant must be $l$-independent. Therefore, rules (i) (ii) apply.

The derivation of the large- $N$ limit is proceeded first by normalizing $I_{2}$ to $N$. In this case, because

$$
I_{2}=f^{2} \sum_{l, m, n}\left(\begin{array}{lll}
j & j & j \\
l & m & n
\end{array}\right)\left(\begin{array}{lll}
l & m & n \\
j & j & j
\end{array}\right)=f^{2}
$$

we may choose $f(N)=\sqrt{N}$. Then the next task is to compute $\alpha_{k}^{i}$.

Amit and Roginsky[24] have considered $N$-component generalizations of the Potts model, which also requires cubic invariants. The cubic invariants were also chosen to be the Wigner $3 j$ coefficients of $\mathrm{SU}(2)$. Using $f(N)=\sqrt{N}$, they computed the conventional $3 m j$ coefficients numerically. In this case, the $3 m j$ coefficients, $(3 m j)$, are related to 2 PI diagrams with $2 m$ vertices by $(3 m j)(\sqrt{N})^{2 m}=I_{2 m}^{(i)}$. They found

$$
|(3 m j)| \lesssim N^{-m+1-\alpha}
$$

with $\alpha>0$ if $m \geq 2$. This implies $I_{2 m}^{(i)}=O\left(N^{1-\alpha}\right)=O\left(N^{1-\alpha_{m}^{i}}\right)$. Hence, $\alpha_{m}^{i}=\alpha>0$ if $m \geq 2$. As a result, when $N$ goes to infinity, only fully $2 \mathrm{PR}$ diagrams survive. 


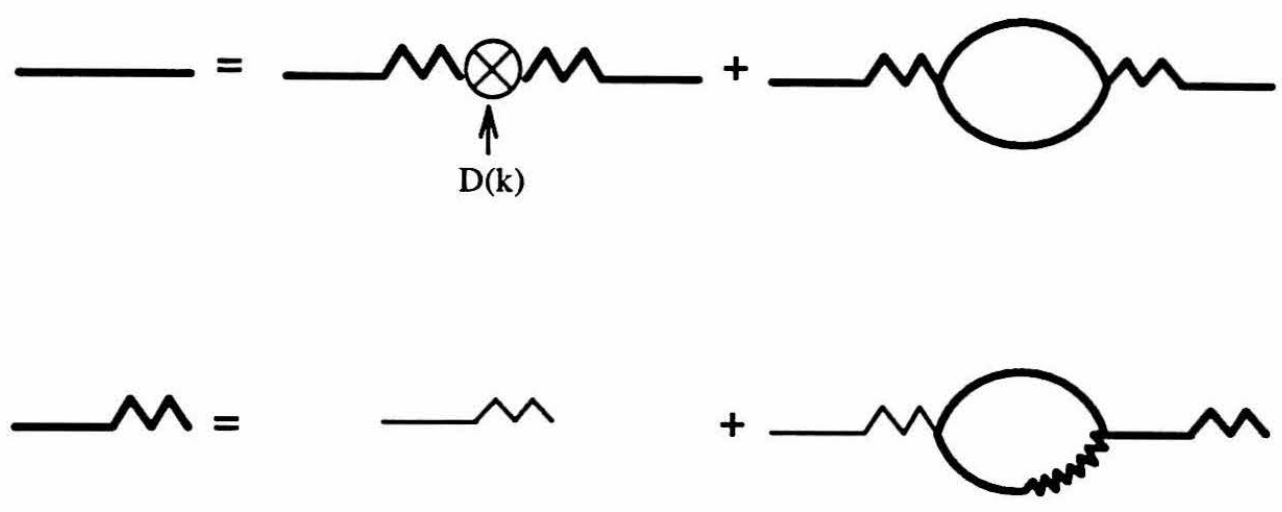

Figure 3-3: Diagrammatic equations in the spherical limit

\subsection{The Resulting Equations}

The main feature of fully $2 \mathrm{PR}$ diagrams is that they consist of bubble diagrams only. In the spherical limit, the two-point correlation functions are simply summations of these bubble diagrams. Let us represent $\hat{U}$ by a thick, straight line and $\hat{G}$ by a thick, straight-wavy line in the spherical limit. Obviously, they satisfy the diagrammatic equations as shown in Figure 3-3, where as usual, thin, straight-wavy lines represent $\hat{G}_{0}$. Using the fact that both $\hat{U}$ and $\hat{G}$ carry the transverse projection operator $\hat{\tau}_{\alpha \beta}(\mathbf{k})$, we may write out these equations. We find

$$
\begin{aligned}
& \frac{1}{\hat{G}(\mathbf{k}, \omega)}=-i \omega+\nu_{0} k^{2}+\lambda_{0} k^{2} \int_{q} \int_{\Omega} b(\mathbf{k}, \mathbf{q}) \hat{U}(\mathbf{k}-\mathbf{q}, \omega-\Omega) \hat{G}(\mathbf{q}, \Omega) \\
& \hat{U}(\mathbf{k}, \omega)=|\hat{G}(\mathbf{k}, \omega)|^{2}\left[D(\mathbf{k}, \omega)+\lambda_{0} k^{2} \int_{q} \int_{\Omega} a(\mathbf{k}, \mathbf{q}) \hat{U}(\mathbf{k}-\mathbf{q}, \omega-\Omega) \hat{U}(\mathbf{q}, \Omega)\right],
\end{aligned}
$$


where $a(\mathbf{k}, \mathbf{q})$ and $b(\mathbf{k}, \mathbf{q})$ result from contractions among $P_{\alpha \beta \gamma}$ 's and $\hat{\tau}_{\alpha \beta}$ 's :

$$
\begin{aligned}
& a(\mathbf{k}, \mathbf{q})=\frac{1}{2 k^{2}} \frac{1}{d-1} P_{i j l}(\mathbf{k}) \hat{\tau}_{j m}(\mathbf{k}-\mathbf{q}) \hat{\tau}_{l n}(\mathbf{q}) P_{i m n}(\mathbf{k})=a\left(\hat{k}, \frac{\mathbf{q}}{k}\right), \\
& b(\mathbf{k}, \mathbf{q})=\frac{1}{k^{2}} \frac{1}{d-1} P_{i j l}(\mathbf{k}) \hat{\tau}_{j m}(\mathbf{k}-\mathbf{q}) P_{l m i}(\mathbf{q})=b\left(\hat{k}, \frac{\mathbf{q}}{k}\right) .
\end{aligned}
$$

Hence $a$ and $b$ are homogeneous functions. In three dimension, they become

$$
\begin{aligned}
& a(\mathbf{k}, \mathbf{q})=\frac{1}{2}\left[1-2 \frac{(\mathbf{k} \cdot \mathbf{q})^{2}(\mathbf{k} \cdot(\mathbf{k}-\mathbf{q}))^{2}}{k^{4} q^{2}(\mathbf{k}-\mathbf{q})^{2}}+\frac{(\mathbf{k} \cdot \mathbf{q})(\mathbf{k} \cdot(\mathbf{k}-\mathbf{q}))(\mathbf{q} \cdot(\mathbf{k}-\mathbf{q}))}{k^{2} q^{2}(\mathbf{k}-\mathbf{q})^{2}}\right] \\
& b(\mathbf{k}, \mathbf{q})=\frac{q}{k}\left[-\frac{(\mathbf{q} \cdot(\mathbf{k}-\mathbf{q}))(\mathbf{k} \cdot(\mathbf{k}-\mathbf{q}))}{k q(\mathbf{k}-\mathbf{q})^{2}}+\frac{(\mathbf{k} \cdot \mathbf{q})^{3}}{k^{3} q^{3}}\right]
\end{aligned}
$$

Also, they satisfy a simple relation,

$$
a(\mathbf{k}, \mathbf{q})=\frac{1}{2}[b(\mathbf{k}, \mathbf{q})+b(\mathbf{k}, \mathbf{k}-\mathbf{q})]
$$

which is true in any dimension and can be verified straightforwardly. It is important to note that, as promised, the driving function $D(\mathbf{k}, \omega)$ is completely arbitrary in (3.4). Therefore, $y$ is a free parameter, and the detailed crossover between short- and long-ranged $D(k)$ can be elucidated now!

These equations are very well known in the theory of turbulence: they are Kraichnan's Direct Interaction Approximation (DIA) equations[14], originally derived through an uncontrolled approximation scheme. We have derived these equations in a well-defined large- $N$ limit. 
The DIA equations are non-linear integral equations which presumably include the energy cascading phenomenon. That is, even though $D(\mathbf{k}, \omega)$ vanishes in the inertial range, $\hat{U}(\mathbf{k}, \omega)$ does not vanish because of the presence of the second term in (3.4). In fact, if we consider the strictly bounded force

$$
D(\mathbf{k}, \omega)\left\{\begin{array}{l}
=0 \text { if } k>m_{0} \\
\neq 0 \text { if } 0 m_{0} \geq k \geq 0
\end{array}\right.
$$

then look at these bubble diagrams that compose $\hat{U}$; one can easily see that the diagram with one bubble vanishes for $k>2 m_{0}$, that diagrams with two nested bubbles vanish for $k>3 m_{0}$, and so on. Therefore, looking at the inertial range $\left(k \gg m_{0}\right)$ effectively picks up diagrams with very large numbers of bubbles, which are the largeorder terms in perturbation theory. The progression of energy towards larger $k$ then corresponds to the progression of the value of $\hat{U}$ towards diagrams with larger numbers of bubbles. This is the way that the DIA equations preserve the energy cascading picture.

We conclude this chapter by a brief discussion of some old attempts at constructing large- $N$ models of turbulence. The most serious attempts of which we are aware are those of so-called stochastic models[17,25,26]. These models, originated from Kraichnan's Random Coupling Model (R.C.M.)[25], are only variations of R.C.M. Kraichnan noted that the DIA equations also result from the large- $N$ limit of the Random Coupling Model in which $A_{N}^{l m n}$ are randomly $\pm \frac{1}{N}$, restricted only by sym- 
metry under permutation of the indices. We stress[17], however, that because these models do not include some higher-order symmetry varying with $N$ and, also, they do not reduce to the original Navier-Stokes equations at $N=1$, we do not expect that they can yield a systematic expansion of the universal exponents. Kraichnan's result does, however, lead one to expect that the limit $N \rightarrow \infty$ will be rather insensitive to the detailed procedure for obtaining it. 


\section{Chapter 4}

\section{Scaling Properties of the Spherical Limit}

We now turn to a complete analysis of the DIA equations. Most of the formalism described in this chapter is carried over from that of a toy model developed in Appendix B. We refer readers to Appendix B for more details.

\subsection{Recovery of the RG's Results and the Analysis of Convergence}

We first note that the DIA equations reproduce the $y$-expansion results precisely. To see this, we assume the dynamical scaling forms, (1.16) and (1.17), in the inertial range $m_{0} \ll k \ll \Lambda$. We shall consider the limit when the inertial range is expanded to the whole space of $k$ by letting $\Lambda$ go to infinity and $m_{0}$ go to zero. Since $\Lambda \rightarrow \infty$ is the same as $\nu_{0} \rightarrow 0$, we are equivalently considering the limit of infinite Reynolds number.

Because $\Lambda \rightarrow \infty$ is the same as $\nu_{0} \rightarrow 0$, we shall drop $\nu_{0}$, put an upper cutoff $\Lambda$ in every integral, and take $\Lambda$ to $\infty$ at the end. Similarly, we shall put the lower-end-cutoff 
$m_{0}$ in every integral and take $m_{0}$ to zero at the end.

When we substitute the scaling forms, (1.16) and (1.17), into the DIA equations and make the rescalings, $s=\omega / \tilde{\nu} k^{z}, t=\Omega / \tilde{\nu} q^{z}$ and $\mathbf{q} \rightarrow \mathbf{q} / k$, we have

$$
\begin{aligned}
\frac{1}{A_{1} \tilde{\nu} g(s)}= & -i s+ \\
& A_{1} A_{2} k^{d+2-\Delta-z} \int d t \int_{m_{0} / k}^{\Lambda / k} d^{d} \mathbf{q} b(\hat{k}, \mathbf{q}) \frac{1}{|\hat{k}-\mathbf{q}|^{\Delta}} u\left(\frac{s-q^{z} t}{|\hat{k}-\mathbf{q}|^{z}}\right) g(t),
\end{aligned}
$$

and

$$
\begin{aligned}
& \frac{A_{2} u(s)}{\tilde{\nu}\left|A_{1}\right|^{2}|g(s)|^{2}}=\frac{D_{0}}{\tilde{\nu}} k^{\Delta-2 z+4-d-y}+ \\
& {A_{2}}^{2} k^{d+2-\Delta-z} \int d t \int_{m_{0} / k}^{\Lambda / k} d^{d} \mathbf{q} b(\hat{k}, \mathbf{q}) \frac{1}{|\hat{k}-\mathbf{q}|^{\Delta}} \frac{1}{q^{\Delta-z}} u\left(\frac{s-q^{z} t}{|\hat{k}-\mathbf{q}|^{z}}\right) u(t),
\end{aligned}
$$

where the integration limits become $k$ dependent. Obviously, if the two integrals involved are convergent when $\Lambda \rightarrow \infty$ and $m_{0} \rightarrow 0$, they are functions of $s$ only. Since all of the other terms in equations are functions of $s$, it is consistent only when the two exponents in $k$ vanish. We thus obtain

$$
\Delta+z=d+2
$$


and

$$
2 \Delta-z=2 d-2+y
$$

which lead directly to the $y$-expansion solution. This is perhaps not surprising because one may perform the renormalization group $y$-expansion directly on the equations (3.3) and (3.4), and the result is precisely the $O(y)$ recursion relations from Ref. 4. Thus the equations (3.3) and (3.4) are an exact integration of these lowest order recursion relations.

It is important to note that the $y$-expansion solutions are exact to all orders in $y$ if $\lambda_{0}$ is not renormalized. When $N=1$, this is guaranteed by the Ward identity derived from Galilean invariance (see Appendix A). However, when $N \neq 1$, even though Galilean invariance can be generalized in a certain way as shown in Chapter 2, the Lagrangian is not invariant under the generalized Galilean transformation. Hence, there is no corresponding Ward identity in the case when $N \neq 1$; nevertheless, by resorting to a direct graph analysis, one can still prove that $\lambda_{0}$ is not renormalized. We refer readers to Appendix A for further details.

The validity of $y$-expansion solutions depends on the convergence of the two integrals in (4.1) and (4.2). The analysis of the convergence can be proceeded first by taking $q \rightarrow \infty$. Then $\left(s-q^{z} t\right) /|\hat{k}-\mathbf{q}|^{z} \rightarrow-x$, and the relevant integrals in (4.1) and (4.2) are reduced to

$$
\int_{q \gg 1} \frac{b(\hat{k}, \mathbf{q})}{q^{\Delta}} \text { and } \int_{q \gg 1} \frac{a(\hat{k}, \mathbf{q})}{q^{2 \Delta-z}}
$$


which are convergent if $\Delta>d$ and $2 \Delta>d+z$. Next, we take $\mathbf{q} \rightarrow \mathbf{0}$ and thus $\left(s-q^{z} t\right) /|\hat{k}-\mathbf{q}|^{z} \rightarrow s$. In this case, we need to consider the integral

$$
\int_{q \ll 1} \frac{a(\hat{k}, \mathbf{q})}{q^{\Delta-z}}
$$

which is convergent when $\Delta<d+z$. Finally, when considering the limit $\mathbf{q} \rightarrow \hat{k}$, we change variables by substituting: $\bar{x}=\left(s-q^{z} t\right) /|\hat{k}-\mathbf{q}|^{z}$. The integrals involved are then

$$
\int_{q \ll 1} \frac{b(\hat{k}, \hat{k}-\mathbf{q})}{q^{\Delta-z}} \text { and } \int_{q \ll 1} \frac{a(\hat{k}, \hat{k}-\mathbf{q})}{q^{\Delta-z}}
$$

These integrals are also convergent in the range $\Delta<d+z$. The final range that every integral is convergent is $d<\Delta<d+z$ (if $0<z<d$ ). When we combine this with the $y$-expansion solutions, we find that the $y$-expansion solutions are valid in the range $0<y<3$. Furthermore, as we increase $y$ towards $3, \Delta$ approaches $d+z$, which is the boundary when the convergence of $m_{0} \rightarrow 0$ fails. Apparently, when $y>3$, we need to include $m_{0}$ carefully in our analysis. This will be done below.

\subsection{Forcing at $y>3$}

In this section, we shall derive the main consequences of $y>3$, i.e., $\Delta>d+z$. We first generalize the dynamical scaling forms to include $m_{0}$ dependence with

$$
G(w, k)=A_{1} k^{-z} g\left(\frac{w}{\tilde{\nu} k^{z}} ; \frac{k}{m_{0}}\right)
$$


and

$$
U(w, k)=A_{2} k^{-\Delta} u\left(\frac{w}{\tilde{\nu} k^{z}} ; \frac{k}{m_{0}}\right)
$$

where $\lim _{x \rightarrow \infty} g(s ; x) \equiv g(s)$ and $\lim _{x \rightarrow \infty} u(s ; x) \equiv u(s)$ are the asymptotic scaling functions in the limit $m_{0} \rightarrow 0$ or, equivalently, in the inertial range. When including the $\frac{k}{m_{0}}$ dependence, equations (4.1) and (4.2) become

$$
\begin{aligned}
& \frac{1}{A_{1} \tilde{\nu} g(s ; x)}=-i s+ \\
& A_{1} A_{2} k^{d+2-\Delta-z} \int_{\mathbf{q} t} b(\hat{k}, \mathbf{q}) \frac{1}{|\hat{k}-\mathbf{q}|^{\Delta}} u\left(\frac{s-q^{z} t}{|\hat{k}-\mathbf{q}|^{2}} ;|\hat{k}-\mathbf{q}| x\right) g(t ; q x),
\end{aligned}
$$

$$
\begin{aligned}
& \frac{A_{2} u(s ; x)}{\tilde{\nu}\left|A_{1}\right|^{2}|g(s ; x)|^{2}}=\frac{D_{0}}{\tilde{\nu}} k^{\Delta-2 z} \eta(x)+ \\
& A_{2}{ }^{2} k^{d+2-\Delta-z} \int_{\mathbf{q} t} a(\hat{k}, \mathbf{q}) \frac{1}{|\hat{k}-\mathbf{q}|^{\Delta}} \frac{1}{q^{\Delta-z}} u\left(\frac{s-q^{z} t}{|\hat{k}-\mathbf{q}|^{z}} ;|\hat{k}-\mathbf{q}| x\right) u(t ; q x),
\end{aligned}
$$

where we have reparameterized $D(\mathbf{k}, \omega)$ by $D_{0}\left(m_{0}\right) \eta\left(\frac{k}{m_{0}}\right)$.

Let us define

$$
\begin{aligned}
J_{a}(s ; x) & \equiv \int_{\mathbf{q} t} a(\hat{k}, \mathbf{q}) \frac{1}{|\hat{k}-\mathbf{q}|^{\Delta}} \frac{1}{q^{\Delta-z}} u\left(\frac{s-q^{z} t}{|\hat{k}-\mathbf{q}|^{z}} ;|\hat{k}-\mathbf{q}| x\right) u(t ; q x), \\
J_{b}(s ;, x) & \equiv \int_{\mathbf{q} t} b(\hat{k}, \mathbf{q}) \frac{1}{|\hat{k}-\mathbf{q}|^{\Delta}} u\left(\frac{s-q^{z} t}{|\hat{k}-\mathbf{q}|^{z}} ;|\hat{k}-\mathbf{q}| x\right) g(t ; q x),
\end{aligned}
$$

and proceed in the same way as what is done in Appendix B to isolate the singularities 
in $J_{a}$ and $J_{b}$ by defining

$$
\begin{aligned}
J_{a}^{\text {sing }}(s ; x) & =u(s ; x) \int_{\mathbf{q} t} 2 a\left(\hat{k}, \frac{\hat{k}-\mathbf{q}}{|\hat{k}-\mathbf{q}|}\right) \frac{1}{|\hat{k}-\mathbf{q}|^{\Delta}} u\left(\frac{s-q^{z} t}{|\hat{k}-\mathbf{q}|^{z}} ;|\hat{k}-\mathbf{q}| x\right), \\
J_{b}^{\text {sing }}(s ;, x) & =g(s ; x) \int_{\mathbf{q} t} b(\hat{k}, \mathbf{q}) \frac{1}{|\hat{k}-\mathbf{q}|^{\Delta}} u\left(\frac{s-q^{z} t}{|\hat{k}-\mathbf{q}|^{z}} ;|\hat{k}-\mathbf{q}| x\right)
\end{aligned}
$$

where we have set $\mathbf{q}$ to $\hat{k}$ in all nonsingular terms. The factor of 2 in $J_{a}^{s i n g}$ arises because $a(\hat{k}, \mathbf{q})=a(\hat{k}, \hat{k}-\mathbf{q})$, so the singularity at $\mathbf{q} \rightarrow \mathbf{0}$ is identical to that at $\mathbf{q} \rightarrow \hat{k}$. By construction, $\Delta J_{a}(s ;, x) \equiv J_{a}(s ;, x)-J_{a}^{s i n g}(s ; x)$ and $\Delta J_{b}(s ; x) \equiv J_{b}(s ;, x)-$ $J_{b}^{s i n g}(s ; x)$ are convergent as $x \rightarrow \infty$ so that we are able to define

$$
\begin{aligned}
& \lim _{x \rightarrow \infty} \Delta J_{a}(s ;, x) \equiv \Delta J_{a}(s), \\
& \lim _{x \rightarrow \infty} \Delta J_{b}(s ;, x) \equiv \Delta J_{b}(s) .
\end{aligned}
$$

By changing variables: $\mathbf{p}=s(\hat{k}-\mathbf{q})$ and $\omega=\left(s-q^{z} t\right) /|\hat{k}-\mathbf{q}|^{z}$, we may write

$$
\begin{aligned}
J_{a}^{s i n g}(s ; x) & =u(s ; x) \int_{\mathbf{p}} 2 a\left(\hat{k}, \frac{\mathbf{p}}{x}\right) \frac{u_{\infty}(p)}{p^{\Delta-z}} x^{\Delta-z-d} \\
& =u(s ; x) \int_{\mathbf{p}}\left[1-(\hat{k} \cdot \mathbf{p})^{2}\right] \frac{u_{\infty}(p)}{p^{\Delta-z}} x^{\Delta-z-d}+O\left(x^{\Delta-z-d-2}\right) \\
& =\frac{d-1}{d} \int_{\mathbf{p}} \frac{u_{\infty}(p)}{p^{\Delta-z}} u(s ; x) x^{\Delta-z-d}+O\left(x^{\Delta-z-d-2}\right), \\
J_{b}^{\text {sing }}(s ; x) & =g(s ; x) \int_{\mathbf{p}} b\left(\hat{k}, \hat{k}-\frac{\mathbf{p}}{x}\right) \frac{u_{\infty}(p)}{p^{\Delta-z}} x^{\Delta-z-d} \\
& =g(s ; x) \int_{\mathbf{p}}\left[1-(\hat{k} \cdot \mathbf{p})^{2}\right] \frac{u_{\infty}(p)}{p^{\Delta-z}} x^{\Delta-z-d}+O\left(x^{\Delta-z-d-2}\right) \\
& =\frac{d-1}{d} \int_{\mathbf{p}} \frac{u_{\infty}(p)}{p^{\Delta-z}} g(s ; x) x^{\Delta-z-d}+O\left(x^{\Delta-z-d-2}\right),
\end{aligned}
$$


where $u_{\infty}(p)$ is defined to be $\int_{-\infty}^{\infty} \frac{d \omega}{2 \pi} u(\omega ; p)$, and we have made use of (B.13) and (B.14).

By using all of the above expressions, the DIA equations (4.5) and (4.6) now read

$$
\begin{aligned}
\frac{1}{A_{1} \tilde{\nu} g(s ; x)}= & -i s+ \\
& A_{1} A_{2} m_{0}{ }^{d+2-\Delta-z} x^{2-2 z}\left[u_{0} g(s ; x)+x^{d+z-\Delta} \Delta J_{b}(s)+\cdots\right], \\
\frac{A_{2} u(s ; x)}{\tilde{\nu}\left|A_{1}\right|^{2}|g(s ; x)|^{2}}= & \frac{D_{0}}{\tilde{\nu}} m_{0}{ }^{\Delta-2 z} x^{\Delta-2 z} \eta(x)+ \\
& A_{2}{ }^{2} m_{0}{ }^{d+2-\Delta-z} x^{2-2 z}\left[u_{0} u(s ; x)+x^{d+z-\Delta} \Delta J_{a}(s)+\cdots\right],
\end{aligned}
$$

where we have defined $u_{0} \equiv \frac{d-1}{d} \int_{\mathbf{p}} \frac{u_{\infty}(p)}{p^{\Delta-z}}$, which may also be rewritten as

$$
u_{0}=\frac{d-1}{d} \frac{m_{0}^{\Delta-d-z}}{A_{2} \tilde{\nu}} \int_{\omega} \int_{\mathbf{k}} \hat{U}(\mathbf{k}, \omega)
$$

Hence $u_{0}$ is a cutoff dependent measure of the total energy density.

We see from (4.7) and (4.8) that we may choose $A_{1} \tilde{\nu}=1$ (take $A_{1}$ to be real) and $u_{0} A_{1} A_{2} m_{0}{ }^{d+2-z-\Delta}=1$. And also, for large $\mathrm{x}$, we may expand

$$
\begin{aligned}
& g(s ;, x)=g(s)+g_{1}(s) x^{d+z-\Delta}+\cdots, \\
& u(s ;, x)=u(s)+u_{1}(s) x^{d+z-\Delta}+\cdots .
\end{aligned}
$$


Clearly, considering the leading terms of (4.7), we require $2-2 z=0$, i.e, $z=1$ and

$$
\frac{1}{g(s)}=-i s+g(s)
$$

Therefore, $g(s)=\left(i s \pm \sqrt{4-s^{2}}\right) / 2$. In order that $g(s) \rightarrow 0$ for large $s$, we choose

$$
g(s)=\frac{i s-\sqrt{4-s^{2}}}{2}
$$

On the other hand, considering the extreme case when $y$ is very large, i.e., $\eta(x)=0$ for $x>1$, comparison of the leading terms in (4.8) yields

$$
\frac{A_{2}}{A_{1}} u(s)=\frac{A_{2}}{A_{1}} u(s)|g(s)|^{2} .
$$

Since $|g(s)|^{2}=1$ only for $s^{2}<4$, we have $u(s)=0$ for $s^{2}>4$ but is otherwise undetermined at this order.

At next-to-leading order, we find

$$
-\frac{g_{1}(s)}{g(s)^{2}}=\frac{\Delta J_{b}(s)}{u_{0}}+g_{1}(s)
$$

and

$$
\frac{1}{|g(s)|^{2}}\left[u_{1}(s)-2 u(s) \operatorname{Re}\left(\frac{g_{1}(s)}{g(s)}\right)\right]=\frac{\Delta J_{a}(s)}{u_{0}}+u_{1}(s) .
$$


From these equations, we can solve $u(s)$ for $s^{2}<4$. We get

$$
-\frac{2 u(s)}{\sqrt{4-s^{2}}} \operatorname{Re}\left[\Delta J_{b}(s)\right]=\Delta J_{a}(s)
$$

Writing this out, we have

$$
\begin{aligned}
& \Delta J_{b}(s)=\int_{\mathbf{q}, t} u\left(\frac{s-q^{z} t}{|\hat{k}-\mathbf{q}|^{2}}\right)|\hat{k}-\mathbf{q}|^{-\Delta}\left[b(\hat{k}, \mathbf{q}) g(t)-\tilde{b}\left(\hat{k}, \frac{\hat{k}-\mathbf{q}}{|\hat{k}-\mathbf{q}|}\right) g(s)\right], \\
& \Delta J_{a}(s)=\int_{\mathbf{q}, t} u\left(\frac{s-q^{z} t}{|\hat{k}-\mathbf{q}|^{z}}\right)|\hat{k}-\mathbf{q}|^{-\Delta}\left[a(\hat{k}, \mathbf{q}) u(t) q^{z-\Delta}-2 \tilde{a}\left(\hat{k}, \frac{\hat{k}-\mathbf{q}}{|\hat{k}-\mathbf{q}|}\right) u(s)\right] .
\end{aligned}
$$

Since $-\frac{2 u(s)}{\sqrt{4-s^{2}}} \operatorname{Re}\left[J_{b}^{s i n g}\right]=J_{a}^{s i n g}$, the final equation for $u(s)$ is

$$
\begin{aligned}
0= & \int_{\mathbf{q}, t} b(\hat{k}, \mathbf{q}) u\left(\frac{s-q^{z} t}{|\hat{k}-\mathbf{q}|^{z}}\right)|\hat{k}-\mathbf{q}|^{-\Delta} \times \\
& \left\{u(s) \sqrt{1-\frac{t^{2}}{4}} \theta\left(1-\frac{t^{2}}{4}\right)-\sqrt{1-\frac{s^{2}}{4}} q^{z-\Delta} u(t)\right\},
\end{aligned}
$$

where $\left(s-q^{z} t\right) /|\hat{k}-\mathbf{q}|^{z}<2$, and we have made use of $a(\hat{k}, \mathbf{q})=\frac{1}{2}[b(\hat{k}, \mathbf{q})+b(\hat{k}, \hat{k}-\mathbf{q})]$ and the fact that $J_{a}(s)$ is invariant under the substitutions: $t^{\prime}=\left(s-q^{z} t\right) /|\hat{k}-\mathbf{q}|^{z}$ and $\overrightarrow{q^{\prime}}=\hat{k}-\mathbf{q}$.

An obvious solution to (4.10) is $u(s) \propto \sqrt{1-\frac{s^{2}}{4}}, \Delta=z$ which is the solution guessed by Kraichnan[14]. Clearly, this solution implies $\Delta=1$, which is ruled out by the requirement $\Delta>z+d$.

Another exact solution, which is also readily read off, is $u(s) \propto \delta(s)$. The consis- 
tency condition is

$$
\int_{\mathbf{q}} b(\hat{k}, \mathbf{q}) \frac{1}{|\hat{k}-\mathbf{q}|^{\Delta-z}} \frac{1}{q^{z}}=\int_{\mathbf{q}} b(\hat{k}, \mathbf{q}) \frac{1}{|\hat{k}-\mathbf{q}|^{\Delta-z}} \frac{1}{q^{\Delta-z}}
$$

i.e., $\Gamma_{b}(\Delta-z, z, d)=\Gamma_{a}(\Delta-z, \Delta-z, d)$. Therefore, this solution corresponds precisely to that of the static model studied in Appendix B, with the substitution $\Delta \rightarrow \Delta-z$. The word "static" is justified since in the time space $u(t)$ is constant. Apparently, this solution is also not the one we are seeking.

Besides the above two trivial solutions, there seems to be no obvious analytic solution. Therefore, numerical work is in order to find $u(s)$ and $\Delta$. Before describing our numerical results, it is convenient to consider the case when $y$ is greater than three, but not very large. To this end, we put $\eta(x)=x^{4-d-y}$ back. Then equation (4.8) becomes

$$
\begin{aligned}
& \frac{A_{2} u(s ; x)}{\tilde{\nu}\left|A_{1}\right|^{2}|g(s ; x)|^{2}}=\frac{D_{0}}{\tilde{\nu}} m_{0}{ }^{\Delta-2 z} x^{\Delta-2 z+4-d-y}+ \\
& A_{2}{ }^{2} m_{0}{ }^{d+2-\Delta-z}\left[u_{0} u(s ; x) x^{2-2 z}+\Delta J_{a}(s) x^{d+2-z-\Delta}+\cdots\right] .
\end{aligned}
$$

Following the same reasoning at the end of Appendix B, we expect that when $y$ is greater than 3 but less than certain $y_{c}, x^{2-2 z}$ is the leading term on the right hand side, while the remaining terms, $x^{\Delta-2 z+4-d-y}$ and $x^{d+2-z-\Delta}$, are both the next-toleading terms. As a consequence, $\Delta$ is $d+\frac{y-1}{2}$ when $3<y<y_{c}$ and sticks to $\Delta\left(y_{c}\right)$ beyond $y_{c}$. On the other hand, (4.10) becomes 


$$
\begin{aligned}
\delta_{0}= & \int_{\mathbf{q}, t} b(\hat{k}, \mathbf{q}) u\left(\frac{s-q t}{|\hat{k}-\mathbf{q}|}\right)|\hat{k}-\mathbf{q}|^{-\Delta} \times \\
& {\left[u(s)\left(\frac{1-t^{2} / 4}{1-s^{2} / 4}\right)^{\frac{1}{2}} \theta\left(1-t^{2} / 4\right)-q^{1-\Delta} u(t)\right], }
\end{aligned}
$$

where $\delta_{0}=\tilde{\nu}^{-3} u_{0}^{2} D_{0} m_{0}^{4-d-y}$, which vanishes at $y_{c}$. It is interesting to note that the reason why turbulence happens at $\delta_{0}=0$ actually reflects the definition of turbulence, in which $D_{0}$ vanishes in the inertial range.

Our numerics for solving (4.11) is described in Appendix C. Figure 4-1 shows $u(s)$ for various $y$ 's with the normalization $u(0)=1$. In Figure $4-2$, we see that $\delta_{0}$ seems to vanish exactly at $y_{c}=4$. This is of course not a proof. If $y_{c}=4$ is exact, we have $z=1, \Delta=d+3 / 2$, and $\zeta=3 / 2$, which agree with Kaichnan's guessed solutions[14]. In Figure 4-3, we show the turbulence scaling function $u(s)$ at different dimensions. Figure 4-4 shows the deviation of $u(s)$ from Kraichnan's guessed solution, $\sqrt{1-s^{2} / 4}$. The deviation is quite large.

\subsection{Viscous Cutoff in the Spherical Limit}

In this section, we discuss the viscous cutoff $\Lambda$, which is an important length scale in Kolmogorov's theory and also plays an important role in subsequent experiments[27]. In Kolmogorov's theory, $\Lambda$ is $\left(\bar{\epsilon} / \nu_{0}^{3}\right)^{1 / 4}$ and $\Lambda / m_{0} \sim(R e)^{3 / 4}$, where $R e$ is the 


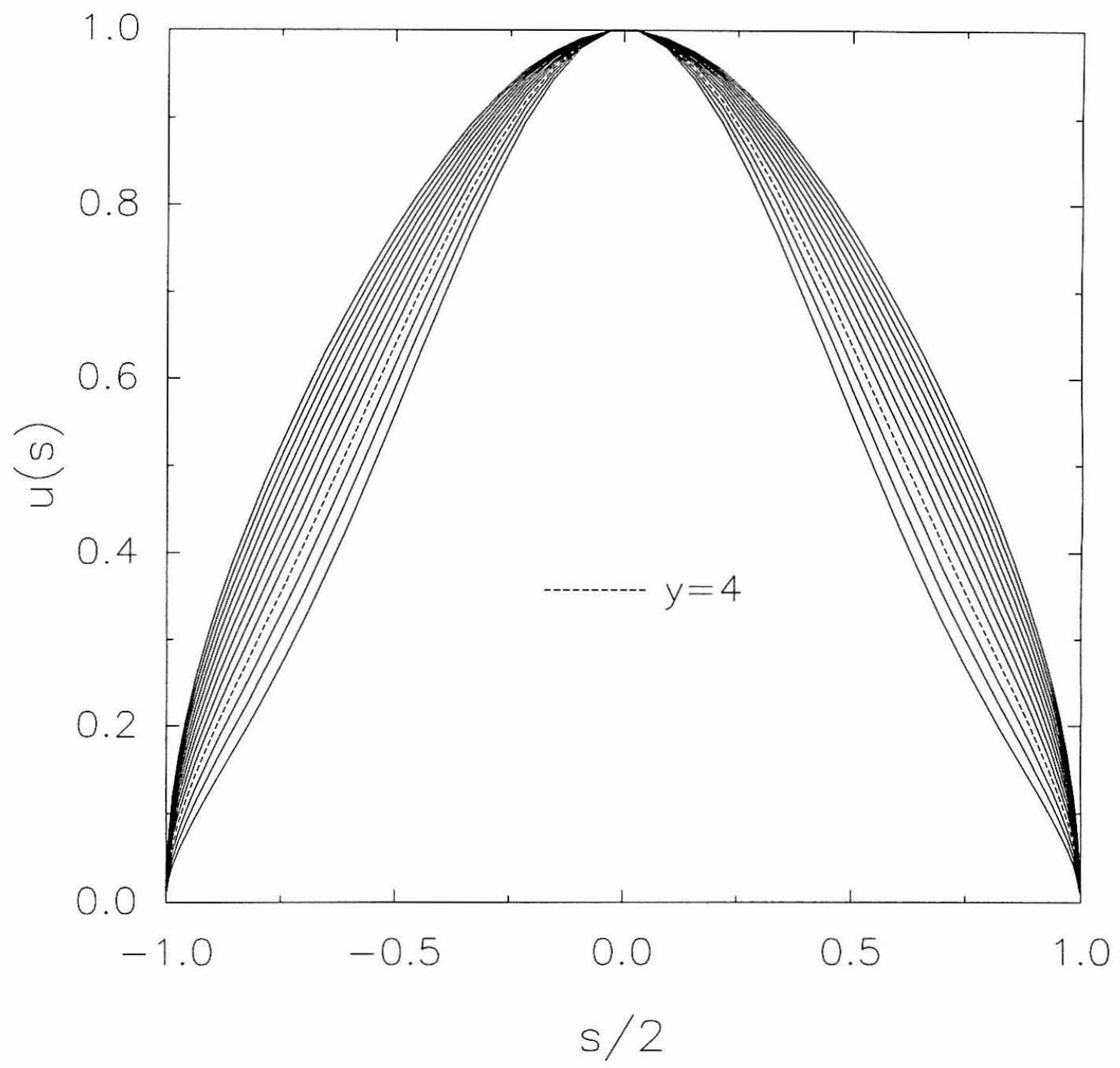

Figure 4-1: Scaling function $u(s)$ for different $y$ 's with $u(0)=1 . y$ is 3.1 for the outmost curve and increases 0.1 for each successive curve. 


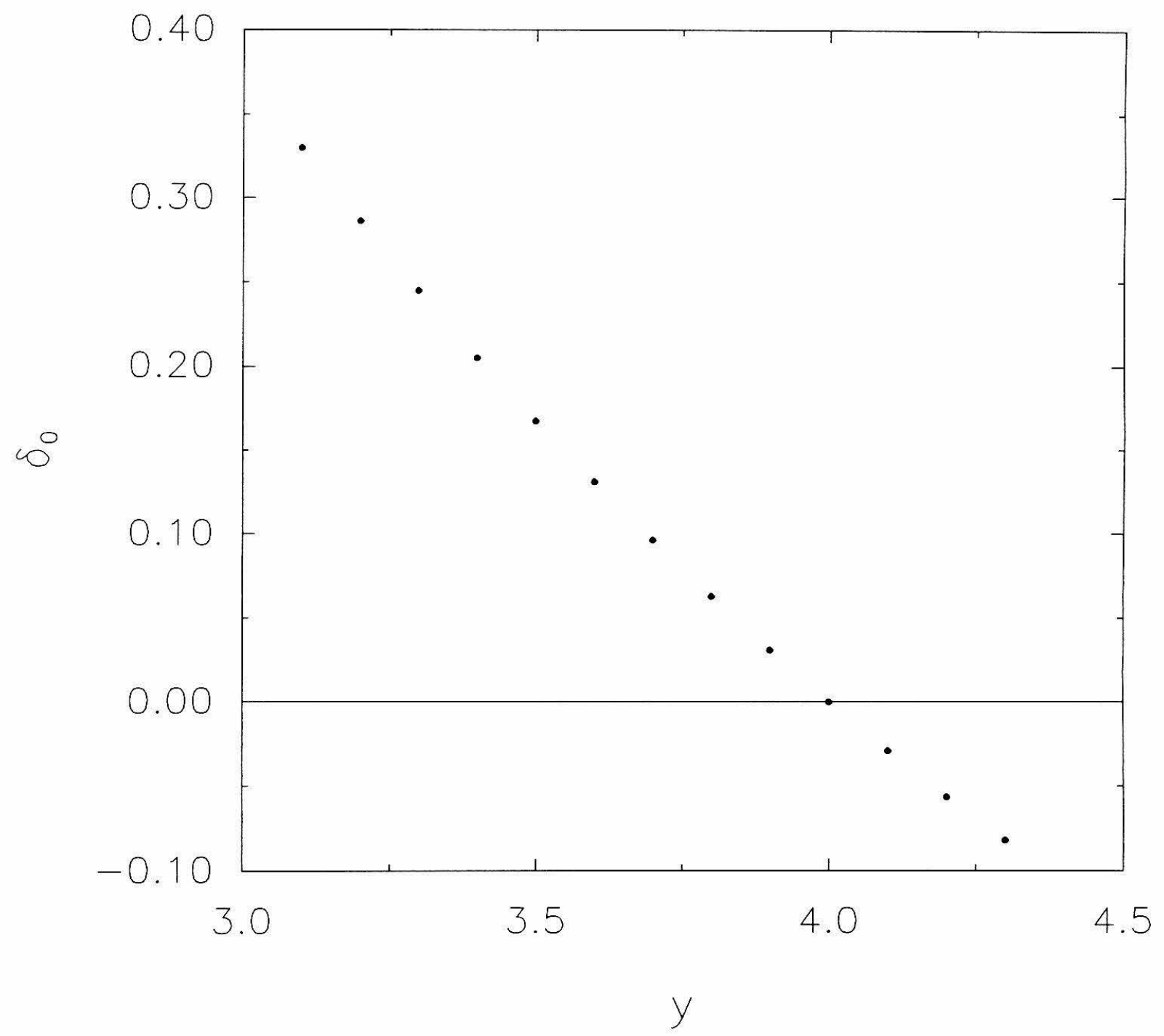

Figure 4-2: $\delta_{0}(y)$ vs. $y$ 


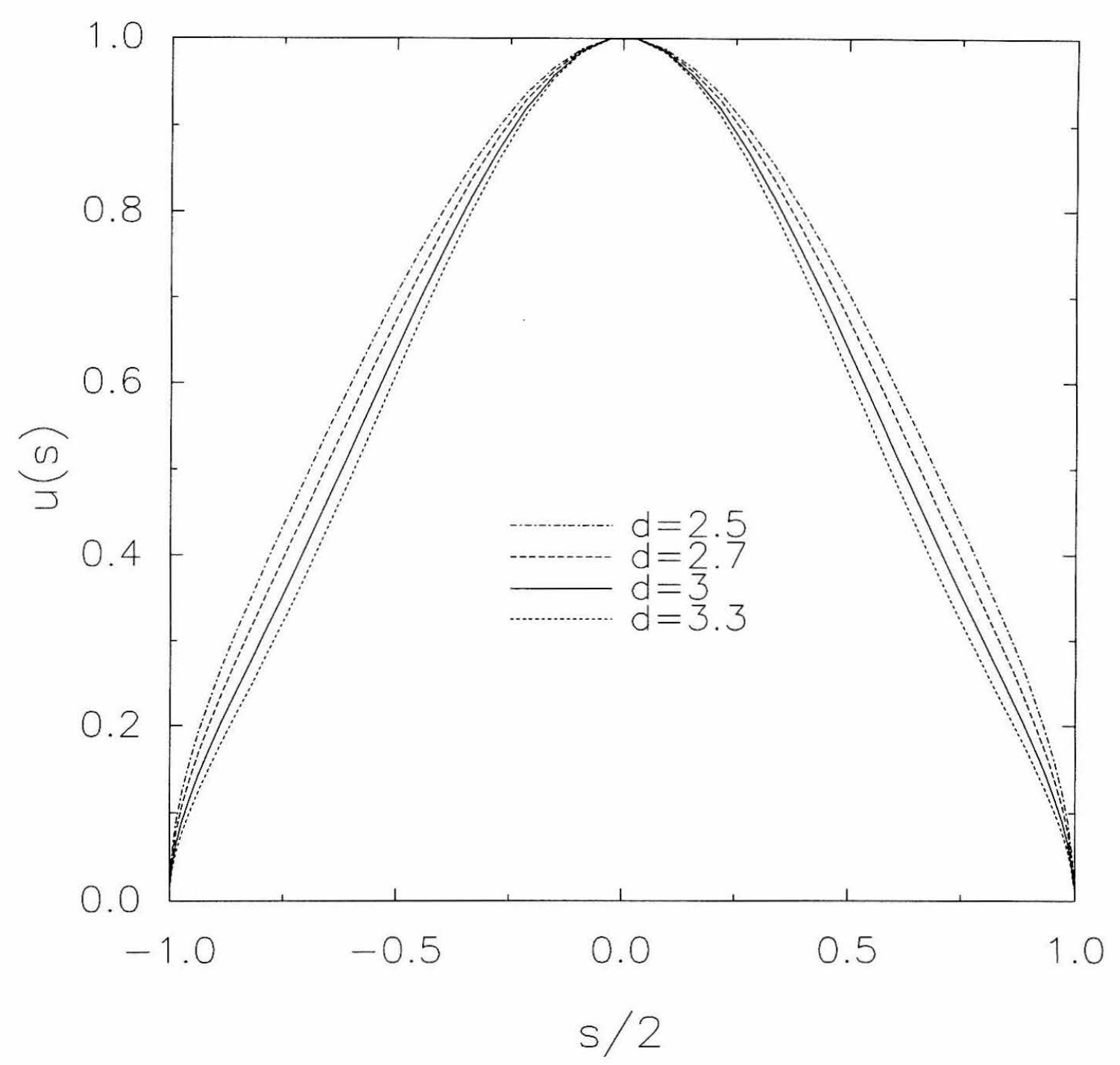

Figure 4-3: Turbulent scaling function for various dimensions 


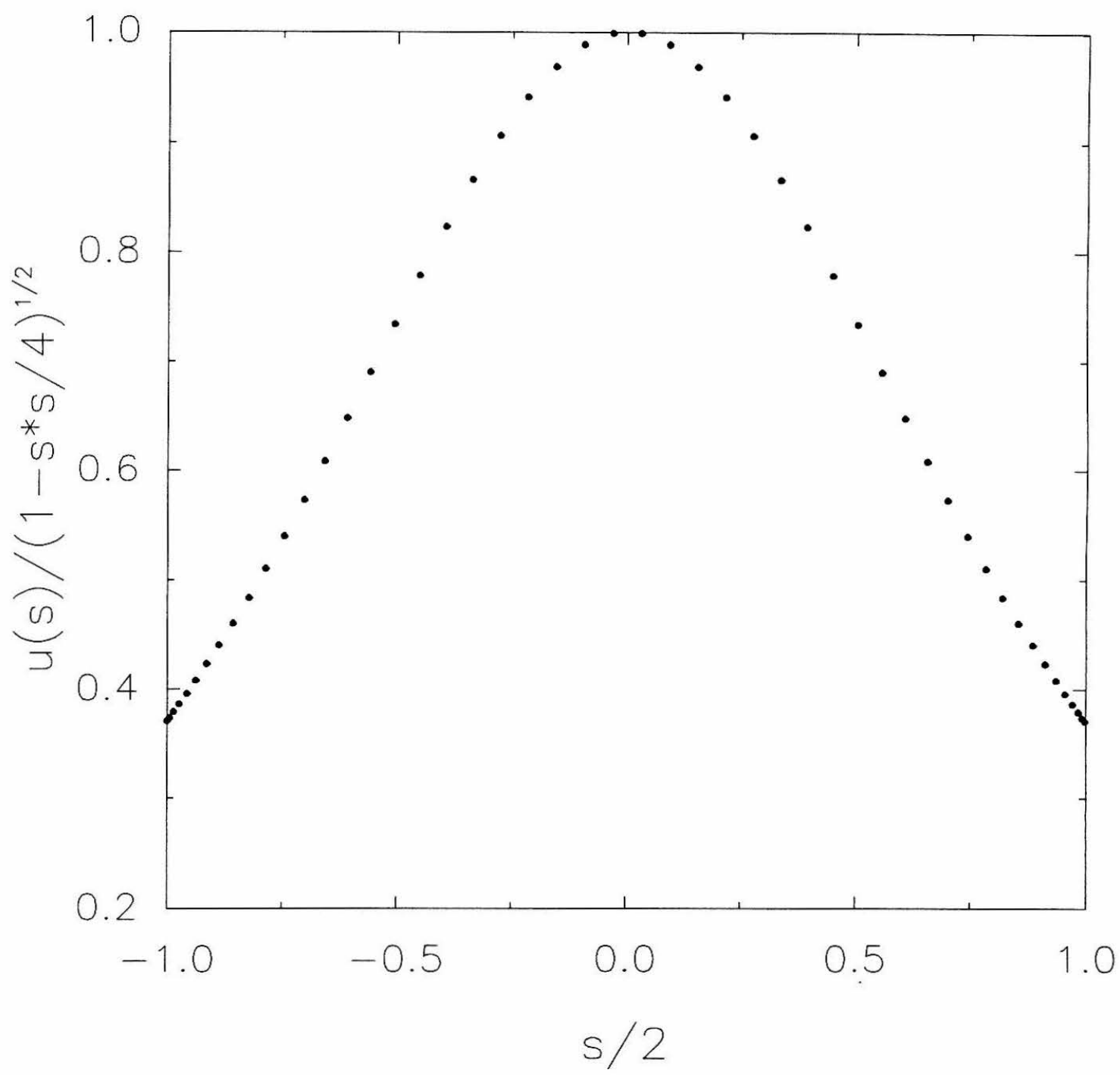

Figure 4-4: Deviation of the correct solution from Kraichnan's solution 
Reynolds number. When including $\Lambda$, the energy spectrum is predicted to be

$$
E(k)=\bar{\epsilon}^{\frac{2}{3}} k^{-\frac{5}{3}} F(k / \Lambda),
$$

where $F(x)$ is a universal function. We may also rewrite it as

$$
\frac{E(k)}{\bar{\epsilon}^{2 / 3} \Lambda^{-5 / 3}}=\left(\frac{k}{\Lambda}\right)^{-5 / 3} F\left(\frac{k}{\Lambda}\right)
$$

Since $\bar{\epsilon}^{2 / 3} \Lambda^{-5 / 3}=\bar{\epsilon}^{1 / 4} \nu_{0}^{5 / 4}$, we may measure $E(k)$ in units of $\bar{\epsilon}^{1 / 4} \nu_{0}^{5 / 4}$ and $k$ in units of $\Lambda$ to test (4.12). If (4.12) is right, one should be able to see that data due to different preparations of turbulence collapse on a universal curve. If neither $k$ nor $E(k)$ are measured in appropriate units, collapse would not occur. Although many subsequent measurements (see Ref. 2) claimed that $F(x)$ is universal, there were also measurements[28] claiming that the data were also consistent with models considering intermittency, in which $m_{0}$ is present in $E(k)$ (which also happens in our spherical limit), and, therefore, $F(x)$ is no longer universal. There appears to be no consensus as to the extent that $F(x)$ is universal. Therefore, it is still an open question.

Despite the important historic role that $\Lambda$ has played in collapsing data, its ratio to $m_{0}$, i.e., $\Lambda / m_{0}$, also gives an estimate of the total number of degrees of freedom in turbulence. Because $\omega \sim k^{z}$, we may naively estimate $\Lambda$ by setting $\nu_{0} \Lambda^{2} \sim \Lambda^{z}$. Hence $\Lambda \sim \nu_{0}{ }^{-\frac{1}{2-z}}$ and, therefore, $\Lambda / m_{0} \sim(R e)^{\frac{1}{2-z}}$. When $z=2 / 3$, we have $\Lambda / m_{0} \sim R e^{3 / 4}$. On the other hand, since $z=1$ in the spherical limit, $\Lambda / m_{0} \sim R e$. As we shall show 
below, this naive analysis turns out to be wrong in the spherical limit.

There are two places in which $\nu_{0}$ enters into the theory. First, there is a $\nu_{0} k^{2}$ term. Second, the cutoff, $\Lambda$, of the integrals also depends on $\nu_{0}$. In general, one expects $\Lambda \rightarrow \infty$ as $\nu_{0} \rightarrow 0$, even though $\Lambda$ may not be proportional to $\nu_{0}^{-3 / 4}$, which is expected in Kolmogorov theory. As far as $\Lambda$ is concerned, its main effect is to renormalize various quantities. For example, if the integral in (3.3) is divergent as $\Lambda \rightarrow \infty$, it contributes a term, $b(\Lambda) k^{2}$, which renormalizes $\nu_{0}$. The fact that we are considering the region where no divergence arises due to $\Lambda$ simply means the effects of renormalization are small.

To see the effect of $\nu_{0} k^{2}$, we include it in (4.7) by

$$
\begin{aligned}
\frac{1}{A_{1} \tilde{\nu} g(s ; x)}= & -i s+\frac{\nu_{0}}{\nu} x^{2-z}+ \\
& A_{1} A_{2} m_{0}{ }^{d+2-\Delta-z} x^{2-2 z}\left[u_{0} g(s ; x)+x^{d+z-\Delta} \Delta J_{b}(s)+\cdots\right],
\end{aligned}
$$

where we have chosen $\tilde{\nu}=\nu m_{0}{ }^{2-z}$ so that $\nu$ has the same dimension of $\nu_{0}$. As was done in Section 4.2, we may choose $z=1, A_{1} \tilde{\nu}=1$ and $u_{0} A_{1} A_{2} m_{0}{ }^{d+2-z-\Delta}=1$. We may also set $A_{2}=1$ and absorb the amplitude into the scaling function $u(s)$. This amplitude of $u(s)$, which is denoted by $\lambda$ in Appendix C, is basically determined by the total energy, i.e., $\lambda \sim u_{0}$ (see equation (C.4)). Hence, we have $A_{1}=\nu^{-1} m_{0}^{z-2}$ 
and $A_{1} m_{0}{ }^{d+2-\Delta-z} u_{0}=1$. Eliminating $A_{1}$, we get

$$
u_{0} / \nu=m_{0}^{\Delta-d}
$$

With the above choices, (4.13) becomes

$$
\frac{1}{A_{1} \tilde{\nu} g(s ; x)}=-i s+g(s ; x)+\frac{1}{u_{0}} x^{d+2-\Delta-z} \Delta J_{b}(s)+\frac{\nu_{0}}{\nu} x^{2-z}+\cdots
$$

Obviously, competing between $\frac{1}{u_{0}} x^{d+2-\Delta-z} \Delta J_{b}(s)$ and $\frac{\nu_{0}}{\nu} x^{2-z}$ sets a characteristic scale, $x_{\Lambda}$, defined by

$$
\frac{\nu_{0}}{\nu} x_{\Lambda}{ }^{2-z} \Delta J_{b}(s)=\frac{\nu_{0}}{\nu} x_{\Lambda}{ }^{2-z}
$$

The relation $\Delta J_{b}(s)=O(u(s)) \sim \lambda \sim u_{0}$ implies

$$
x_{\Lambda}=\frac{\Lambda}{m_{0}} \sim\left(\frac{\nu}{\nu_{0}}\right)^{\frac{1}{\Delta-d}} .
$$

To make contact with the real experimental parameter, $R e$, we make use of equation (4.9) and find

$$
u_{0} \sim \frac{m_{0}^{\Delta-d-2}}{\nu}\left\langle\mathrm{v}^{2}\right\rangle
$$

Eliminating $u_{0}$ in (4.14) and (4.16), we get $\nu \sim m_{0}^{-1} v=l_{0} v$. Therefore, $\nu / \nu_{0}$ is 
simply the Reynolds number. As a consequence, we get

$$
\frac{\Lambda}{m_{0}} \sim(R e)^{\frac{1}{\Delta-d}} \sim R e^{2 / 3} .
$$

This is the correct prediction for $\Lambda / m_{0}$ in spherical limit. 


\section{Chapter 5}

\section{Summary and Future Work}

In summary, we have proposed and investigated a spherical model for turbulence. Our results in the spherical limit are summarized in Figures 1-2 and 1-3. As $y$ is less than three, the exponents follow the RG's results. When $y$ exceeds three, the RG's results were demonstrated to be invalid. Specifically, when $y>3$, we find that $z$ first sticks to one at $y=3$ and then $\Delta$ sticks to $d+3 / 2$ at $y=4$, while $\Delta$ is still controlled by the driving force between $y=3$ and $y=4$.

One of the areas that the formalism developed in this thesis may become useful is in the theories of surface growth. The model that attracted most attention is the Kardar-Parisi-Zhang[29] (KPZ) equation of which the non-linear interaction is also cubic. In the lowest-order RG calculations, however, the non-linear coupling becomes intractably large at $d=2$; and hence, the correct exponents and scaling functions are not known. This is quite similar to the situation of turbulence in which the whole scalings are also dominated by the non-linear coupling. Application of our formalism also produces a pair of coupled integral equations. We expect that a similar numerical 
work on these equations can provide some insights into what is going on at $d=2$.

The other work that needs to be addressed in the future is the next order corrections to the spherical limit. We have made a brief attempt at going beyond the spherical limit. Amit and Roginsky's numerical work suggests that the next order terms would be the order of $1 / \sqrt{N}$. These terms that contribute $O(1 / \sqrt{N})$ are $(6 j)$ and $(9 j)$ coefficients, where the $(6 j)$ coefficients are the second term shown in Figure $3-2$, and the $(9 j)$ coefficients are obtained by adding two more vertices to the $(6 j)$ coefficients. There are seven different diagrams that one has to add to the DIA equations in considering the $(6 j)$ coefficients, and there are even more diagrams for the $(9 j)$ coefficients. Analyzing these extra diagrams is a daunting task, but seems to be a necessary further step.

We close by a brief discussion of the Taylor frozen hypothesis. As it was mentioned, the physics behind $z=1$ may be summarized in the Taylor frozen hypothesis. The Taylor frozen hypothesis is usually invoked when measuring the energy spectrum $E(k)$. It was originally introduced to analyze grid turbulence with a mean flow speed[30]. In this case, the hypothesis reduces the measurement of $E(k)$, which needs to be done at different spatial points, to the measurement of the frequency spectrum $\tilde{E}(\omega)$ at one fixed spatial point. If the mean flow speed is $U$, one may simply replace $\omega$ in $\tilde{E}(\omega)$ by $k \cdot U$ to get $E(k)$.

In the absence of a mean flow, it is expected[31] that the flow caused by large eddies act effectively as the local mean flow, so the Taylor frozen hypothesis still 
applies locally except that $U$ is substituted by $\left\langle\mathbf{v}(\mathbf{r}, t)^{2}\right\rangle^{1 / 2}$. Because the local mean flow is randomly distributed, this effect is usually termed the "random sweeping effect." This effect is especially pronounced when the difference between the spinning velocity scale of large eddies and that of the small eddies is large. In other words, it is expected to happen only at high Reynolds number.

People have argued that because of the random sweeping effect, the small scale structures of turbulence change very little when they pass by a fixed spatial point; therefore, it is impossible to observe the internal dynamics of the small scale structures by looking at the frequency spectrum at a fixed spatial point. Much effort has been thus directed to remove the sweeping effect. The most frequently referred models are those in which one adopts the Lagrangian type description of fluids by following the fluid particles in certain ways[15]. Usually, these theories are not only too complicated for rigorous analysis, even at the level of the DIA equations, but also too cumbersome and insufficiently systematic for further expansions to higher order terms. At the level of the DIA equations, they focus on producing extra terms, which cancel the divergent parts of the integrals when $y \geq 3$, to show the exactness of the Kolmogorv $\frac{5}{3}$-law. As mentioned, the danger for doing this is that if these same terms are produced when $y<3$, there will be large- $k$ divergences in the region described by the $y$-expansion. The theory will then fail to encompass the known exact results.

In a word, all of these attempts are motivated by the closeness of experimental data to $-5 / 3$, and the main efforts are to show that the Komogorov's assumptions can 
be realized in the Lagrangian type descriptions. Once the Komogorov's assumptions are realized, the sweeping effects can be removed completely and therefore all of the predictions made by Komogorov are correct; in particular, the $-5 / 3$ is exact. In view of the experimental data for the higher-order spetra of turbulence, there is no evidence that Kolmogorv's predictions are correct[37]. These theories will then fail to give correct higher-order spectra of turbulence.

The idea we propose in this thesis is to face the infrared divergences caused by the sweeping effect directly. To this end, we have proposed a new set of generalized models for turbulence, parameterized by the the number of velocity fields, $\mathrm{N}$. The DIA equations represent an exact solution in a special limit, which is continuously related, via $N$, to the real turbulence problem. These equations should thus be taken at face value. Previous work[15] which has concentrated on modifying them to obtain the $\frac{5}{3}$-law thus appears to miss the mark. We view the $\frac{3}{2}$-law not as a problem to be fixed, but rather as an amazingly accurate zeroth order approximation in a systematic expansion for $\zeta(N)$. The closeness of the experimental value to the Kolmogorov- $\frac{5}{3}$ is perhaps an unfortunate coincidence which has led people away from taking the DIA equations as seriously as they deserve. 


\section{Appendix A}

\section{The Ward Identity and the Renormalization of $\lambda_{0}$}

The exactness of $\zeta(y)=1-2 y / 3$ when $0<y<3$ is closely related to Galilean invariance at $N=1$. Here, following the usual method employed in Quantum Field theory, we present the derivation of the Ward identity[4b] resulting from Galilean invariance at $N=1$. The main consequence of the Ward identity is that $\lambda_{0}$ is not renormalized[4b]. We further analyze this consequence through a direct graph analysis, which generalizes the method of Forster, Nelson and Stephen[4a]. Finally, we show that even though the Lagrangian is not invariant under the generalized Galilean transformation derived in Section 2.6 when $N>1$ (hence there is no corresponding Ward identity); nevertheless, this graph analysis allows us to show that $\lambda_{0}$ is also not renormalized, even when $N \neq 1$. 


\section{A.1 The Ward Identity $(N=1)$}

To derive the Ward identity, we introduce source terms into the Lagrangian $\mathcal{L}[\mathbf{v}, \mathbf{w}]$ and define

$$
Z\left[\mathbf{J}_{1}, \mathbf{J}_{\mathbf{2}}\right]=\int D \mathbf{v} \int D \mathbf{w} e^{\mathcal{L}[\mathbf{v}, \mathbf{w}]+\int d t \int d^{d} r\left(\mathbf{J}_{\mathbf{1}} \cdot \mathbf{v}+\mathbf{J}_{2} \cdot \mathbf{w}\right)}
$$

One can also define a "free energy" by $F\left[\mathbf{J}_{1}, \mathbf{J}_{2}\right] \equiv-\ln Z\left[\mathbf{J}_{1}, \mathbf{J}_{2}\right]$ and then perform a Legendre transformation of $F\left[\mathbf{J}_{1}, \mathbf{J}_{2}\right]$ via:

$$
\Gamma[\overline{\mathbf{v}}, \overline{\mathbf{w}}]=F\left[\mathbf{J}_{\mathbf{1}}, \mathbf{J}_{\mathbf{2}}\right]+\int d t \int d^{d} r\left(\mathbf{J}_{\mathbf{1}} \cdot \overline{\mathbf{v}}+\mathbf{J}_{\mathbf{2}} \cdot \overline{\mathbf{w}}\right),
$$

and

$$
\overline{\mathbf{v}}=-\frac{\delta F}{\delta \mathbf{J}_{\mathbf{1}}}=\langle\mathbf{v}\rangle, \quad \overline{\mathbf{w}}=-\frac{\delta F}{\delta \mathbf{J}_{\mathbf{2}}}=\langle\mathbf{w}\rangle
$$

It is clear that $\overline{\mathbf{v}}$ and $\overline{\mathbf{w}}$ vanish when $\mathbf{J}_{\mathbf{1}}=\mathbf{J}_{\mathbf{2}}=0$.

The Galilean transformation on various fields are:

$$
\begin{aligned}
& \mathbf{v}^{\prime}(\mathbf{r}, t)=\mathbf{v}\left(\mathbf{r}+\lambda_{0} \mathbf{u} t, t\right)-\mathbf{u} \\
& \overline{\mathbf{v}}^{\prime}(\mathbf{r}, t)=\overline{\mathbf{v}}\left(\mathbf{r}+\lambda_{0} \mathbf{u} t, t\right)-\mathbf{u}, \\
& \mathbf{a}^{\prime}(\mathbf{r}, t)=\mathbf{a}\left(\mathbf{r}+\lambda_{0} \mathbf{u} t, t\right),
\end{aligned}
$$

where a can be any fields of $\mathbf{w}, \overline{\mathbf{w}}, \mathbf{J}_{\mathbf{1}}$ or $\mathbf{J}_{\mathbf{2}}$. Under these transformations, one finds

$$
\mathcal{L}\left[\mathbf{v}^{\prime}, \mathbf{w}^{\prime}\right]=\mathcal{L}[\mathbf{v}, \mathbf{w}]
$$




$$
\begin{aligned}
F\left[\mathbf{J}_{\mathbf{1}}{ }^{\prime}, \mathbf{J}_{\mathbf{2}}{ }^{\prime}\right] & =F\left[\mathbf{J}_{\mathbf{1}}, \mathbf{J}_{\mathbf{2}}\right]+\int d t \int d^{d} \mathbf{J}_{\mathbf{1}} \cdot \mathbf{u}, \\
\Gamma\left[\overline{\mathbf{v}}^{\prime}, \overline{\mathbf{w}}^{\prime}\right] & =\Gamma[\overline{\mathbf{v}}, \overline{\mathbf{w}}] .
\end{aligned}
$$

Therefore, $\delta \Gamma=0$.

Considering the infinitesimal Galilean transformation when $\mathbf{u}=\epsilon \mathbf{h} t$ and $\epsilon \ll 1$, we get from $O(\epsilon)$ of $\delta \Gamma$ that

$$
0=\int d t \int d^{d} r\left\{\frac{\delta \Gamma}{\bar{v}_{\gamma}(\mathbf{r}, t)}\left[\lambda_{0} t \mathbf{h} \cdot \nabla \bar{v}_{\gamma}(\mathbf{r}, t)-h_{\gamma}\right]+\frac{\delta \Gamma}{\bar{w}_{\gamma}(\mathbf{r}, t)} \lambda_{0} t \mathbf{h} \cdot \nabla \bar{w}_{\gamma}(\mathbf{r}, t)\right\}
$$

Integrating by parts, this becomes

$$
0=\int d t \int d^{d} r\left\{\left[\lambda_{0} t \mathbf{h} \cdot \nabla \frac{\delta \Gamma}{\bar{v}_{\gamma}(\mathbf{r}, t)}\right] \bar{v}_{\gamma}(\mathbf{r}, t)+h_{\gamma} \frac{\delta \Gamma}{\bar{v}_{\gamma}(\mathbf{r}, t)}+\left[\lambda_{0} t \mathbf{h} \cdot \nabla \frac{\delta \Gamma}{\bar{w}_{\gamma}(\mathbf{r}, t)}\right] \bar{w}_{\gamma}(\mathbf{r}, t)\right\}
$$

Now functionally differentiating this equation twice with respect to $\bar{v}_{\beta}\left(\mathbf{r}_{1}, t_{1}\right)$ and $\bar{w}_{\alpha}\left(\mathbf{r}_{2}, t_{2}\right)$, and taking $\mathbf{J}_{\mathbf{1}}=\mathbf{J}_{\mathbf{2}}=0$, so that $\overline{\mathbf{v}}=\overline{\mathbf{w}}=0$, we obtain

$$
\begin{aligned}
0= & \lambda_{0}\left(t_{2}-t_{1}\right) \mathbf{h} \cdot \nabla_{r_{2}} \frac{\delta^{2} \Gamma}{\delta \bar{v}_{\beta}\left(\mathbf{r}_{1}, t_{1}\right) \delta \bar{w}_{\alpha}\left(\mathbf{r}_{2}, t_{2}\right)}+ \\
& \int d t \int d^{d} r h_{\gamma} \frac{\delta^{3} \Gamma}{\delta \bar{v}_{\gamma}(\mathbf{r}, t) \delta \bar{v}_{\beta}\left(\mathbf{r}_{1}, t_{1}\right) \delta \bar{w}_{\alpha}\left(\mathbf{r}_{2}, t_{2}\right)}
\end{aligned}
$$

The first term on the right hand side is related to the response function by

$$
\frac{\delta^{2} \Gamma}{\delta \bar{v}_{\beta}\left(\mathbf{r}_{1}, t_{1}\right) \delta \bar{w}_{\alpha}\left(\mathbf{r}_{2}, t_{2}\right)}=\tau_{\alpha \beta} G^{-1}\left(\mathbf{r}_{2}-\mathbf{r}_{1}, t_{2}-t_{1}\right)
$$


The content of (A.1) becomes clear when we apply the following Fourier transform:

$$
\int \frac{d^{d} r_{1}}{(2 \pi)^{d}} \int \frac{d^{d} r_{2}}{(2 \pi)^{d}} \int \frac{d t_{1}}{2 \pi} \int \frac{d t_{2}}{2 \pi} e^{i \mathbf{k} \cdot \mathbf{r}_{2}+i \mathbf{p} \cdot \mathbf{r}_{1}-i \omega t_{2}-i \Omega t_{1}}
$$

We find that

$$
\lambda_{0} \mathbf{h} \cdot \mathbf{k} \tau_{\alpha \beta}(\mathbf{k}) \frac{\partial}{\partial \omega} \hat{G}^{-1}(\mathbf{k}, \omega)+h_{\gamma} \Gamma_{\boldsymbol{\alpha} \beta \gamma}(\mathbf{k} \omega ;-\mathbf{k}-\omega, \mathbf{0} 0)=0
$$

where $\Gamma_{\alpha \beta \gamma}$ is the three-point vertex function. Since $\mathbf{h}$ is arbitrary, we have

$$
\lambda_{0} k_{\gamma} \tau_{\alpha \beta}(\mathbf{k}) \frac{\partial}{\partial \omega} \hat{G}^{-1}(\mathbf{k}, \omega)+\Gamma_{\alpha \beta \gamma}(\mathbf{k} \omega ;-\mathbf{k}-\omega, \mathbf{0} 0)=0
$$

When the bare vertex is symmetrized with respect to $\alpha$ and $\beta$, i.e., $\Gamma_{\alpha \beta \gamma}^{0}=\frac{i}{2} \lambda_{0} P_{\alpha \beta \gamma}$, we must also symmetrize the above result. Finally, we have

$$
\frac{1}{2} \lambda_{0} P_{\alpha \beta \gamma}(\mathbf{k}) \frac{\partial}{\partial \omega} \hat{G}^{-1}(\mathbf{k}, \omega)+\Gamma_{\alpha \beta \gamma}(\mathbf{k} \omega ;-\mathbf{k}-\omega, \mathbf{0} 0)=0
$$

Equation (A.2) is the Ward identity we seek, which can be trivially verified to the order of $\lambda_{0}$. 


\section{A.2 Direct Graph Analysis}

As mentioned, the consequence of (A.2) is that $\lambda_{0}$ is not renormalized; this is because $\frac{\partial}{\partial \omega} \hat{G}^{-1}(\mathbf{k}, \omega)$ is convergent at $y=0[4 \mathrm{~b}]$. To see this result more directly, we resort to direct graphical analysis. First, by naive power counting, we realize that the primitive divergences for the $(2 \mathrm{n}+1)^{t h}$ order diagram of $\Gamma_{\alpha \beta \gamma}$ are $\Lambda^{-n y}$, where we have set all of the internal wavenumbers to $\Lambda$ and all of the internal frequencies to $\Lambda^{2}$. Therefore, all of the diagrams of $\Gamma_{\alpha \beta \gamma}$ are logarithmically divergent at $y=0$. This, however, doesn't imply that the overall $\Gamma_{\alpha \beta \gamma}$ will also diverge, because these divergences may as well cancel each other when we sum them up. Indeed, as we will show below, this is what actually happens.

Following Forster, Nelson, and Stephen, we rewrite

$$
\hat{U}_{0}(\mathbf{k}, \omega)=\left|\hat{G}_{0}(\mathbf{k}, \omega)\right|^{2} D_{0} k^{4-d-y}=\frac{D_{0}}{2 \nu_{0}} k^{2-d-y}\left[\hat{G}_{0}(\mathbf{k}, \omega)+\hat{G}_{0}^{*}(\mathbf{k}, \omega)\right]
$$

and replace all straight lines in diagrams by either straight-wavy lines $\left(\hat{G}_{0}\right)$ or wavystraight lines $\left(\hat{G}_{0}^{*}\right)$.

The resulting diagrams have representations in time-space in which $\hat{G}_{0}(\mathbf{k}, t)$ is retarded, and each vertex is associated with a time label. Because $\hat{G}_{0}$ is retarded, the smaller time label is assigned to the straight end in every straight-wavy line. Thus, each resulting diagram can be specified by the ordering of time labels on vertices. It is important to note that in resulting diagrams, vertices may carry one, two, or even 
three wavy lines, but no matter how many wavy lines they carry, there is only one for each vertex carrying $\frac{i}{2} P_{\alpha \beta \gamma}$. To make distinctions, we use a thick wavy line for the one carrying $\frac{i}{2} P_{\alpha \beta \gamma}$.

One can easily deduce that given the topology and the time ordering of vertices for a diagram, there is only one way to draw this diagram by using straight-wavy lines only and without making distinctions between thick wavy lines and thin wavy lines. Hence, there is only one vertex whose time label is the largest for each diagram. However, the topology and the time ordering of vertices do not uniquely determine a diagram due to the different orientations of the thick wavy line at each vertex.

Given the topology of the diagrams and the time ordering of the vertices, we classify diagrams according to the orientation of the thick wavy line at each vertex: in each class, the orientations of the thick wavy lines are fixed at all of the vertices except the vertex with the largest time label. Hence, diagrams in the same class differ from each other only by the orientation of the thick wavy line at the vertex with the largest time label. There are two possible situations. First, if the largest time vertex is connected to an external line, there are two possible orientations. These are shown in Figure A-1. The summation of leading divergences of these two possible orientations is

$$
\left[\tilde{P}_{\alpha \beta \gamma}(-\boldsymbol{\Lambda})+\tilde{P}_{\gamma \beta \alpha}(\boldsymbol{\Lambda})\right]\left(\frac{D_{0}}{2 \nu_{0}} \frac{\Lambda^{2-d-y}}{\Lambda^{4}}\right) \frac{1}{\Lambda^{4}} \tau_{\alpha \alpha^{\prime}}(\boldsymbol{\Lambda}) \tau_{\gamma \gamma^{\prime}}(\boldsymbol{\Lambda}) M_{\alpha^{\prime} \gamma^{\prime}}(\boldsymbol{\Lambda})
$$

where $M_{\alpha^{\prime} \gamma^{\prime}}$ is the contribution from the remaining part of the diagrams, $P_{\alpha \beta \gamma}$ is reduced to $\tilde{P}_{\alpha \beta \gamma}(\mathbf{k}) \equiv k_{\gamma} \delta_{\alpha \beta}+k_{\beta} \delta_{\alpha \gamma}$ by using the incompressibility condition, and we 

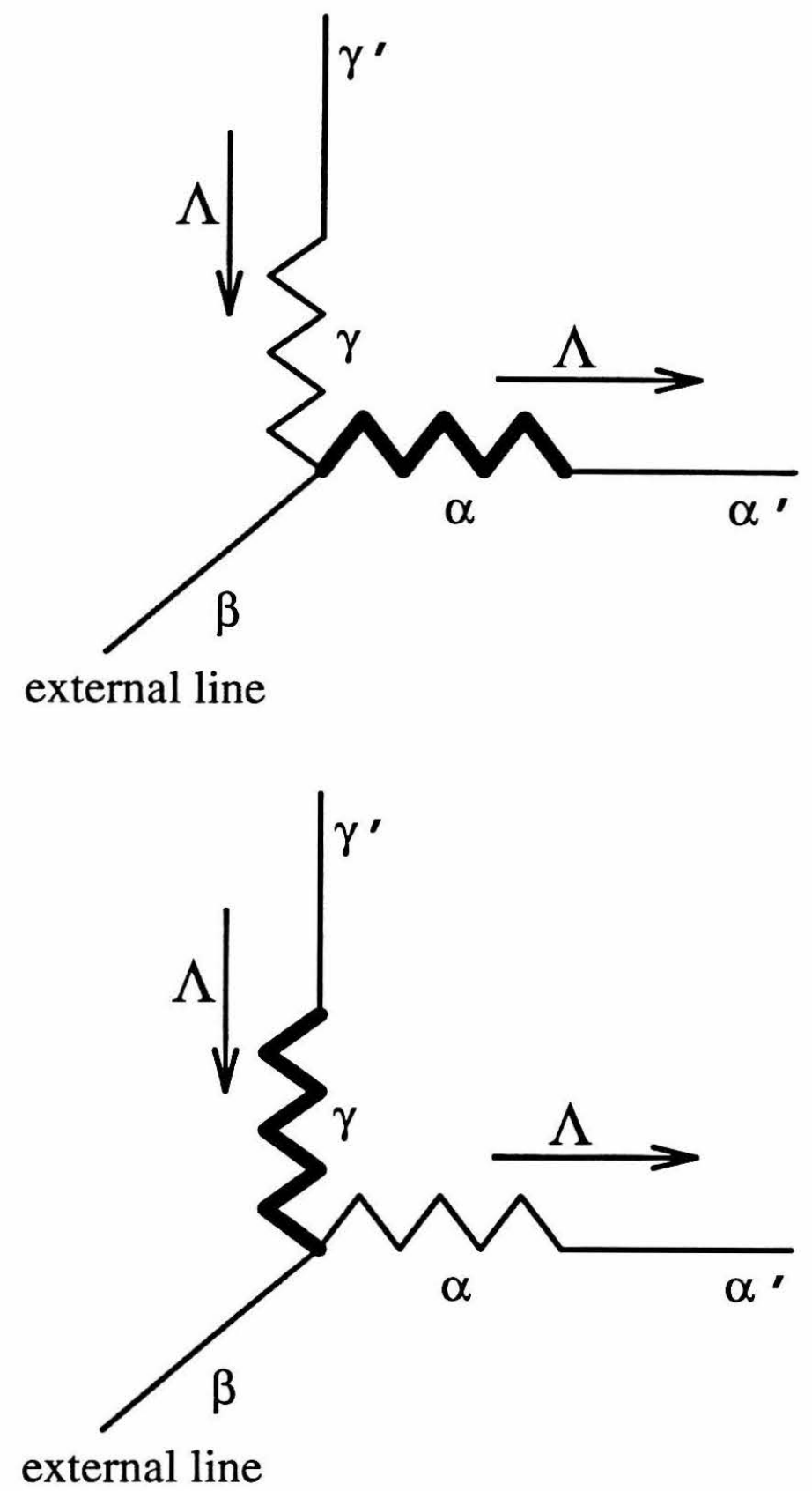

Figure A-1: Two possible orientations when the largest time vertex connects to an external line 
have also made use of $\hat{G}_{0} \sim \frac{1}{\Lambda^{4}}$. It is straightforward to show that the summation in the bracket is zero.

The second possibility occurs when the largest time vertex is entirely internal as shown in Figure A-2. In this case, the summation of leading divergences is

$$
\begin{aligned}
& {\left[\tilde{P}_{\boldsymbol{\alpha} \beta \gamma}\left(\boldsymbol{\Lambda}_{1}\right)+\tilde{P}_{\beta \alpha \gamma}\left(\boldsymbol{\Lambda}_{2}\right)+\tilde{P}_{\gamma \alpha \beta}\left(\boldsymbol{\Lambda}_{3}\right)\right] \times} \\
& \tau_{\alpha \alpha^{\prime}}\left(\boldsymbol{\Lambda}_{1}\right) \tau_{\beta \beta^{\prime}}\left(\boldsymbol{\Lambda}_{2}\right) \tau_{\gamma \gamma^{\prime}}\left(\boldsymbol{\Lambda}_{3}\right) N_{\alpha^{\prime} \beta^{\prime} \gamma^{\prime}}(\boldsymbol{\Lambda})\left(\frac{D_{0}}{2 \nu_{0}} \frac{\Lambda^{2-d-y}}{\Lambda^{4}}\right)^{2} \frac{1}{\Lambda^{4}},
\end{aligned}
$$

where $\Lambda_{1}+\Lambda_{2}+\Lambda_{3}=0,\left|\Lambda_{1}\right|=\left|\Lambda_{2}\right|=\left|\Lambda_{3}\right|=\Lambda$, and $N_{\alpha^{\prime} \beta^{\prime} \gamma^{\prime}}$ denotes the contribution from the rest of diagrams. The total sum in the bracket also vanishes in this case. Therefore, the leading divergences in any class of diagrams get canceled.

Now since the leading divergences are logarithmic at $y=0$ and they are cancelled after summation, we conclude that $\Gamma_{\alpha \beta \gamma}$ is convergent at $y=0$. As a result, $\lambda_{0}$ is not renormalized.

When $N \neq 1$, there is an additional factor due to the presence of $A_{N}^{l m n}$ for each diagram. However, if $A_{N}^{l m n}$ is symmetric under permutations of $l, m$, and $n$, these factors will be the same for diagrams considered in either Figure A-1 or Figure A-2. Hence, the same cancellations happen, and $\lambda_{0}$ is also not renormalized. 

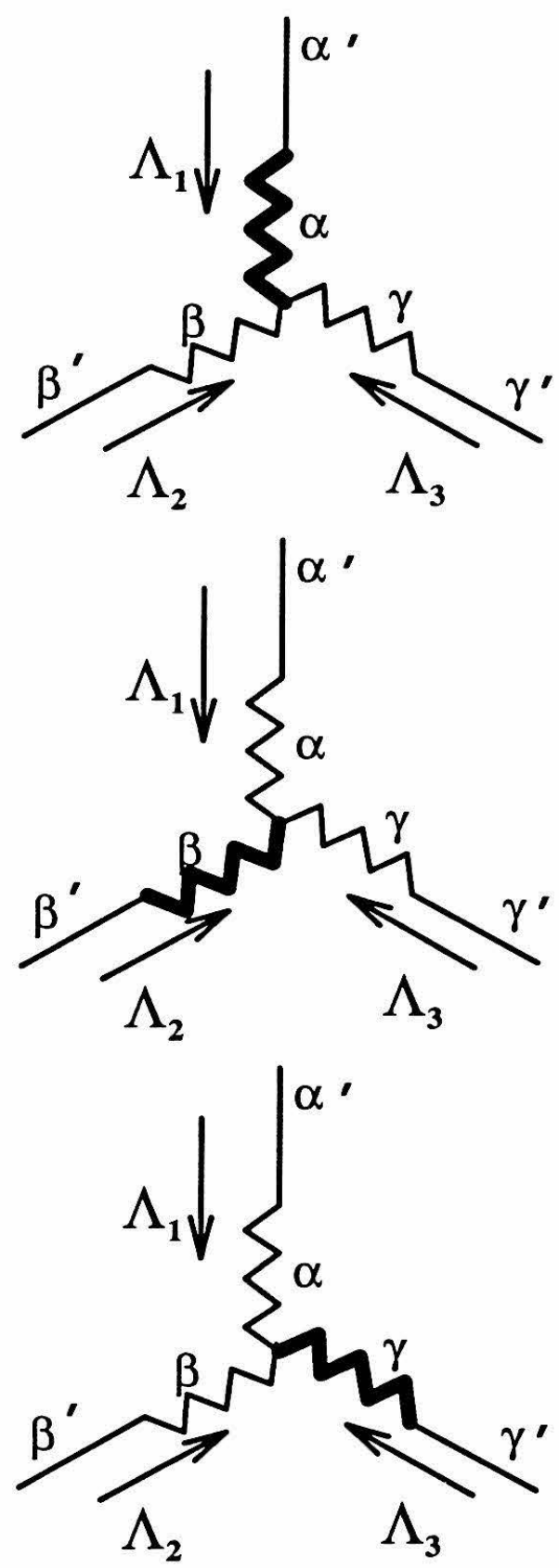

Figure A-2: Three possible orientations when the largest time vertex is internal 


\section{Appendix B}

\section{The Model DIA Equations}

To seek the intuition for analyzing DIA equations, we analyze a set of simplified equations - model DIA equations - in which all frequency dependencies are simply suppressed

$$
\begin{aligned}
\frac{1}{G(\mathbf{k})} & =\nu_{0} k^{2}+k^{2} \int_{\mathbf{q}} b(\mathbf{k}, \mathbf{q}) U(\mathbf{k}-\mathbf{q}) G(\mathbf{q}) \\
U(\mathbf{k}) & =|G(\mathbf{k})|^{2}\left[D(\mathbf{k})+k^{2} \int_{\mathbf{q}} a(\mathbf{k}, \mathbf{q}) U(\mathbf{k}-\mathbf{q}) U(\mathbf{q})\right]
\end{aligned}
$$

The underlying equations are the time-independent Navier-Stokes equations

$$
\lambda_{0}(\mathbf{v} \cdot \nabla) \mathbf{v}=-\frac{1}{\rho_{0}} \nabla p+\nu_{0} \nabla^{2} \mathbf{v}+\mathbf{f} ; \quad \nabla \cdot \mathbf{v}=0
$$

Note that although the dynamics is suppressed, the nontrivial kernels $a(\mathbf{k}, \mathbf{q})$ and $b(\mathbf{k}, \mathbf{q})$ are still included. 
If we reparameterize $D(k)$ by

$$
D(k)=\frac{D_{0} k^{6-d}}{\left(m_{0}^{2}+k^{2}\right)^{\frac{y}{2}}},
$$

then $\left[\lambda_{0}\right]=\frac{y}{2}$ (the dimension of $\lambda_{0}$ ) is the same as before[4b].

To explore the solutions of (B.1) and (B.2), we assume power law solutions

$$
\begin{aligned}
& G(k) \sim \frac{h_{1}}{k^{z}}, \\
& U(k) \sim \frac{h_{2}}{k^{\Delta}} .
\end{aligned}
$$

When we substitute them into (B.1) and (B.2) and rescale $\mathbf{q}$ by $\mathbf{q} / k$, we get

$$
\begin{aligned}
& 1=h_{1}{ }^{2} h_{2} k^{d+2-\Delta-2 z} \int_{m_{0} / k}^{\Lambda / k} d^{d} \mathbf{q} b(\hat{k}, \mathbf{q}) \frac{1}{|\hat{k}-\mathbf{q}|^{\Delta} q^{z}} \\
& 1=D_{0} \frac{h_{1}{ }^{2}}{h_{2}} k^{6-d-y+\Delta-2 z}+h_{1}{ }^{2} h_{2} k^{d+2-\Delta-2 z} \int_{m_{0} / k}^{\Lambda / k} d^{d} \mathbf{q} a(\hat{k}, \mathbf{q}) \frac{1}{|\hat{k}-\mathbf{q}|^{\Delta} q^{\Delta}}
\end{aligned}
$$

Clearly, if the integrals are convergent, we would conclude

$$
\begin{aligned}
\Delta+2 z & =d+2, \\
6-d-y & =2 z-\Delta .
\end{aligned}
$$

Thus, we have $z=2-\frac{y}{4}$ and $\Delta=d-2+\frac{y}{2}$, which play the same role as the $y$-expansion solutions to the full DIA equations. To find when the integrals are convergent, we 
need to know how $a(\hat{k}, \mathbf{q})$ and $b(\hat{k}, \mathbf{q})$ behave as $q \rightarrow \infty, \mathbf{q} \rightarrow \mathbf{0}$, or $\mathbf{q} \rightarrow \hat{k}$. These are summarized below:

(a) $q \rightarrow \infty$

$$
\begin{aligned}
& a(\hat{k}, \mathbf{q})=\frac{1}{d-1} \sin ^{2} \theta\left(d-2+2 \cos ^{2} \theta\right)+O\left(\frac{1}{q}\right) \\
& b(\hat{k}, \mathbf{q})=\frac{2}{d-1} q\left(\cos ^{3} \theta-\cos \theta\right)+\sin ^{2} \theta+O\left(\frac{1}{q}\right),
\end{aligned}
$$

(b) $\mathbf{q} \rightarrow \mathbf{0}$

$$
\begin{aligned}
& a(\hat{k}, \mathbf{q})=\frac{1}{2} \sin ^{2} \theta+O(q) \\
& b(\hat{k}, \mathbf{q})=\frac{2}{d-1}\left(\cos ^{3} \theta-\cos \theta\right) q+\left(\sin ^{2} \theta\right) q^{2}+O\left(q^{3}\right)
\end{aligned}
$$

(c) $q^{\prime} \equiv|\hat{k}-\mathbf{q}| \rightarrow 0$

$$
\begin{aligned}
& a(\hat{k}, \mathbf{q})=a(\hat{k}, \hat{k}-\mathbf{q}), \text { the expansion is the same as }(\mathrm{b}) \\
& b(\hat{k}, \mathbf{q})=\sin ^{2} \theta^{\prime}+\left({\mathbf{q}^{2}}^{2}\right),
\end{aligned}
$$

where $\cos \theta=\hat{k} \cdot \hat{q}$, while $\cos \theta^{\prime}=\hat{k} \cdot \hat{q}^{\prime}$. It is then easy to show that the integrals in (B.5) and (B.6) are convergent as long as $\frac{d}{2}<\Delta<d, \Delta+z>d$ and $z<d+2$. Consequently, the $y$-expansion solutions of the model DIA equations are valid only when $4-d<y<4$. Moreover, as $y \rightarrow 4, \Delta$ approaches $d$, which is the boundary when the convergence of $m_{0} \rightarrow 0$ fails.

It follows from the above observation that when $y>4$, one has to treat the lower cutoff $m_{0}$ carefully. As a first step, we consider an extreme case when $y$ is very large. For this purpose, let us parameterize $D(k)$ by $D_{0}\left(m_{0}\right) \eta\left(\frac{k}{m_{0}}\right)$, with $\eta(x)=0$ when $x>1$. Since everything is convergent as $k \rightarrow \infty$ when $y>4$, the limit $\nu_{0} \rightarrow 0$ is well- 
defined and we may simply drop it. The only relevant scale is then $m_{0}$. Therefore, we generalize (B.3) and (B.4) to

$$
\begin{aligned}
G(k) & =\frac{h_{1}}{k^{z}} g(s), \\
U(k) & =\frac{h_{2}}{k^{\Delta}} u(s),
\end{aligned}
$$

where $s=\frac{k}{m_{0}}$ and by construction, $g(s), u(s) \rightarrow 1$ as $s \rightarrow \infty$. It suffices to take constant $\Delta$ and $z$ in the whole range of $s$, while the forcing dependence and the crossover from $s<1$ to $s>1$ are all thrown into $g(s)$ and $u(s)$. Following the same procedure of obtaining (B.5) and (B.6), we substitute (B.7) and (B.8) into the model DIA equations and find

$$
\begin{aligned}
\frac{1}{g(s)}= & h_{1}{ }^{2} h_{2}\left(m_{0} s\right)^{d+2-\Delta-2 z} \int_{\mathbf{q}} b(\hat{k}, \mathbf{q}) \frac{u(s|\hat{k}-\mathbf{q}|) g(s q)}{|\hat{k}-\mathbf{q}|^{\Delta} q^{z}} \\
\frac{u(s)}{|g(s)|^{2}}= & D_{0}\left(m_{0}\right)\left(m_{0} s\right)^{\Delta-2 z} \frac{h_{1}{ }^{2}}{h_{2}} \eta(s)+ \\
& h_{1}{ }^{2} h_{2}\left(m_{0} s\right)^{d+2-\Delta-2 z} \int_{\mathbf{q}} a(\hat{k}, \mathbf{q}) \frac{u(s|\hat{k}-\mathbf{q}|) u(s q)}{|\hat{k}-\mathbf{q}|^{\Delta} q^{\Delta}} .
\end{aligned}
$$

Here no explicit cutoffs are introduced for the integrals. Singularities due to the limit $m_{0} \rightarrow 0$ appear in the singular behaviors of the integral as functions of $s$.

Clearly, (B.9) and (B.10) imply $h_{1}{ }^{2} h_{2} \sim m_{0}^{\Delta+2 z-d-2}$. Moreover, if $D_{0}\left(m_{0}\right)=$ $D_{0} m_{0}{ }^{-P}$, then $\frac{h_{1}^{2}}{h_{2}} \sim m_{0}{ }^{P+2 z-\Delta}$. Hence, $h_{1} \sim m_{0}{ }^{z+\frac{1}{4}(P-d-2)}$ and $h_{2} \sim m_{0}{ }^{\Delta-\frac{1}{2}(P+d+2)}$. 
Let

$$
\begin{aligned}
I_{a}(s) & \equiv \int_{\mathbf{q}} a(\hat{k}, \mathbf{q}) \frac{u(s|\hat{k}-\mathbf{q}|) u(s q)}{|\hat{k}-\mathbf{q}|^{\Delta} q^{\Delta}} \\
I_{b}(s) & \equiv \int_{\mathbf{q}} b(\hat{k}, \mathbf{q}) \frac{u(s|\hat{k}-\mathbf{q}|) g(s q)}{|\hat{k}-\mathbf{q}|^{\Delta} q^{z}}
\end{aligned}
$$

As it was mentioned, they are convergent when $\frac{d}{2}<\Delta<d, \Delta+z>d$ and $z<d+2$. We shall consider the case when $\Delta<d$ is violated so that only singularities at $\mathbf{q} \rightarrow \mathbf{0}$ and $\mathbf{q} \rightarrow \hat{k}$ are problems. These singularities can be easily isolated because they essentially happen at isolated points. For example, the singularity at $\mathbf{q} \rightarrow \hat{k}$ may be isolated by setting the regular parts (excluding $a$ and $b$ ) of the integrands to their values at $\mathbf{q}=\hat{k}$. Hence, we define

$$
\begin{aligned}
I_{a}^{\text {sing }} & =u(s) \int_{\mathbf{q}} a(\hat{k}, \mathbf{q}) \frac{u(s|\hat{k}-\mathbf{q}|)}{|\hat{k}-\mathbf{q}|^{\Delta}}+u(s) \int_{\mathbf{q}} a(\hat{k}, \mathbf{q}) \frac{u(s q)}{q^{\Delta}} \\
I_{b}^{\text {sing }} & =g(s) \int_{\mathbf{q}} b(\hat{k}, \mathbf{q}) \frac{u(s|\hat{k}-\mathbf{q}|)}{|\hat{k}-\mathbf{q}|^{\Delta}}
\end{aligned}
$$

Therefore, $\Delta I_{a}(s) \equiv I_{a}(s)-I_{a}^{\text {sing }}$ and $\Delta I_{b}(s) \equiv I_{b}(s)-I_{b}^{\text {sing }}$ are convergent. As $s \rightarrow \infty$, since $u(s|\hat{k}-\mathbf{q}|), u(s q)$ and $g(s q) \rightarrow 1$ for most $q$ except when $\mathbf{q} \cong \hat{k}$ or $q \cong 0$, and at these points the integrands are either subtracted or vanishing, we conclude

$$
\Delta I_{a}(\infty) \equiv \Gamma_{a}(\Delta, \Delta, d)=\text { Finite part of } \int_{\mathbf{q}} a(\hat{k}, \mathbf{q}) \frac{1}{|\hat{k}-\mathbf{q}|^{\Delta} q^{\Delta}}
$$




$$
\Delta I_{b}(\infty) \equiv \Gamma_{b}(\Delta, \Delta, d)=\text { Finite part of } \int_{\mathbf{q}} b(\hat{k}, \mathbf{q}) \frac{1}{|\hat{k}-\mathbf{q}|^{\Delta} \mathbf{q}^{z}}
$$

By changing variables: either $\mathbf{w}=s \mathbf{q}$ or $\mathbf{w}=s(\hat{k}-\mathbf{q})$, we may write

$$
\begin{aligned}
& I_{a}^{s i n g}=s^{\Delta-d} u(s) \int_{\mathbf{w}} \frac{u(w)}{w^{\Delta}} 2 a\left(\hat{k}, \frac{\mathbf{w}}{s}\right), \\
& I_{b}^{s i n g}=s^{\Delta-d} g(s) \int_{\mathbf{w}} \frac{u(w)}{w^{\Delta}} b\left(\hat{k}, \hat{k}-\frac{\mathbf{w}}{s}\right),
\end{aligned}
$$

where we have made use of $a(\mathbf{k}, \mathbf{q})=a(\mathbf{k}, \mathbf{k}-\mathbf{q})$. Now because of

$$
\begin{aligned}
a\left(\hat{k}, \frac{\mathbf{w}}{s}\right) & =\frac{1}{2}\left(1-(\hat{k} \cdot \hat{w})^{2}\right)+O\left(\frac{1}{s^{2}}\right), \\
b\left(\hat{k}, \hat{k}-\frac{\mathbf{w}}{s}\right) & =1-(\hat{k} \cdot \hat{w})^{2}+O\left(\frac{1}{s^{2}}\right),
\end{aligned}
$$

we may simply redefine

$$
\begin{aligned}
I_{a}^{\text {sing }} & =u(s) \int_{\mathbf{q}} 2 \tilde{a}\left(\hat{k}, \frac{\hat{k}-\mathbf{q}}{|\hat{k}-\mathbf{q}|}\right) \frac{u(s|\hat{k}-\mathbf{q}|)}{|\hat{k}-\mathbf{q}|^{\Delta}} \\
I_{b}^{\text {sing }} & =g(s) \int_{\mathbf{q}} \tilde{b}\left(\hat{k}, \frac{\hat{k}-\mathbf{q}}{|\hat{k}-\mathbf{q}|}\right) \frac{u(s|\hat{k}-\mathbf{q}|)}{|\hat{k}-\mathbf{q}|^{\Delta}}
\end{aligned}
$$

with $2 \tilde{a}(\hat{k}, \hat{w})=\tilde{b}(\hat{k}, \hat{w}) \equiv 1-(\hat{k} \cdot \hat{w})^{2}$. The leading order terms of $I_{a}(s)$ and $I_{b}(s)$ can thus be identified

$$
\begin{aligned}
& I_{a}(s)=\Gamma_{a}(\Delta, \Delta, d)+u_{\infty} s^{\Delta-d} u(s)+\cdots, \\
& I_{b}(s)=\Gamma_{b}(\Delta, z, d)+u_{\infty} s^{\Delta-d} g(s)+\cdots
\end{aligned}
$$


where $u_{\infty}=\int \frac{u(w)}{w^{\Delta}}\left(1-(\hat{k} \cdot \hat{w})^{2}\right)=\frac{d-1}{d} \int \frac{u(w)}{w^{\Delta}}$ is a cutoff dependent measure of total kinetic energy density. In view of (B.13) and (B.14), it is clear that all the higher order terms are at least $O\left(s^{\Delta-d-2}\right)$, which vanish if $d<\Delta<d+2$.

We now substitute $I_{a}(s)$ and $I_{b}(s)$ back to (B.9) and (B.10). We find

$$
\begin{aligned}
& \frac{1}{g(s)}=h_{1}{ }^{2} h_{2}\left(m_{0} s\right)^{d+2-\Delta-2 z}\left[\Gamma_{b}(\Delta, z, d)+u_{\infty} s^{\Delta-d} g(s)+\cdots\right], \\
& \frac{u(s)}{|g(s)|^{2}}=h_{1}{ }^{2} h_{2}\left(m_{0} s\right)^{d+2-\Delta-2 z}\left[\Gamma_{a}(\Delta, \Delta, d)+u_{\infty} s^{\Delta-d} u(s)+\cdots\right] .
\end{aligned}
$$

For large $s$ and $\Delta>d$, the R.H.S.'s are dominated by the $u_{\infty}$ terms. Thus we require $d+2-\Delta-2 z+\Delta-d=0$, i.e., $z=1$ and $h_{1}{ }^{2} h_{2}\left(m_{0} s\right)^{d-\Delta} u_{\infty}=1$. The equations then become

$$
\begin{aligned}
& \frac{1}{g(s)}=\frac{1}{u_{\infty}} \Gamma_{b}(\Delta, z, d) s^{d-\Delta}+g(s)+\cdots, \\
& \frac{u(s)}{|g(s)|^{2}}=\frac{1}{u_{\infty}} \Gamma_{a}(\Delta, \Delta, d) s^{d-\Delta}+u(s)+\cdots .
\end{aligned}
$$

It is clear that to next-to-leading order, we may write[32]

$$
\begin{aligned}
& g(s)=1+g_{1} s^{d-\Delta}+\cdots, \\
& u(s)=1+u_{1} s^{d-\Delta}+\cdots .
\end{aligned}
$$


Substituting them into (B.17) and (B.18), one finds

$$
g_{1}=-\frac{1}{2 u_{\infty}} \Gamma_{b}(\Delta, z, d)=-\frac{1}{2 u_{\infty}} \Gamma_{b}(\Delta, \Delta, d)
$$

and $u_{1}$ is undetermined at this order. This finally determines the self-consistent equation for the exponent $\Delta: \Gamma_{a}(\Delta, \Delta, d)=\Gamma_{b}(\Delta, z, d)$ and $z=1$.

To solve for $\Delta$, we first note that because $a(\hat{k}, \mathbf{q})=\frac{1}{2}(b(\hat{k}, \mathbf{q})+b(\hat{k}, \hat{k}-\mathbf{q}))$, we have $\Gamma_{a}(\Delta, \Delta, d)=\Gamma_{b}(\Delta, \Delta, d)$. Thus the final equation we need to solve is

$$
\Gamma_{b}(\Delta, \Delta, d)=\Gamma_{b}(\Delta, 1, d)
$$

Let $\hat{k} \cdot \hat{q}=y, b(\hat{k}, \mathbf{q})$ becomes

$$
b(q, y)=\frac{q\left(1-y^{2}\right)\left[\left(d-1+4 y^{2}\right) q-2 y-2 q y^{2}\right]}{(d-1)\left(1-2 q y+q^{2}\right)}
$$

Note that $b(q, y)$ satisfies $b\left(\frac{1}{q}, y\right)=\frac{1}{q^{2}} b(q, y)$. We may write out the integrals in $\Gamma_{b}$, which is the finite part of

$$
I=K_{d-1} \int_{0}^{\infty} d q q^{d-1-z} \int_{-1}^{1} d y\left(1-y^{2}\right)^{\frac{d-3}{2}} \frac{b(q, y)}{\left(1-2 q y+q^{2}\right)^{\frac{\Delta}{2}}}
$$

where $(2 \pi)^{d} K_{d-1}$ is the area of unit sphere in $d-1$ dimension. After doing a simplified 
"conformal transformation" [33] $q \rightarrow \frac{1}{q^{\prime}}$, we get

$$
\begin{aligned}
I & =K_{d-1} \int_{0}^{\infty} d q^{\prime} q^{d-1}{q^{\prime}}^{\Delta+z-2 d-2} \int_{-1}^{1} d y\left(1-y^{2}\right)^{\frac{d-3}{2}} \frac{b\left(q^{\prime}, y\right)}{\left(1-2 q^{\prime} y+q^{\prime 2}\right)^{\frac{\Delta}{2}}} \\
& =\int_{\overrightarrow{q^{\prime}}} b\left(\hat{k}, \overrightarrow{q^{\prime}}\right) \frac{1}{\left|\hat{k}-\overrightarrow{q^{\prime}}\right|^{\Delta} q^{\prime 2 d+2-\Delta-z}} .
\end{aligned}
$$

It implies $\Gamma_{b}(\Delta, z, d)=\Gamma_{b}(\Delta, 2 d+2-\Delta-z, d)$ [34]. An application of this identity is that aside from the obvious solution $\Delta=z$ for (B.19), we may also have $z=$ $2 d+2-2 \Delta$, i.e., $2 \Delta+z=2 d+2$ which yields $z=1$ and $\Delta=d+\frac{1}{2}$. However, since we are interested in the range $\Delta>d, \Delta=z$ is ruled out, and we are left with the only solutions, $z=1$ and $\Delta=d+\frac{1}{2}$, at this stage.

The conformal transformation is not powerful enough to exhaust all possible solutions. To explore other possible solutions, one has to evaluate $\Gamma_{b}$ numerically. A good way to accomplish this evaluation is via using the identity $2 \vec{a} \cdot \vec{b}=(\vec{a}+\vec{b})^{2}-a^{2}-b^{2}$. Then $b(\hat{k}, \mathbf{q})$ can be cast in the form $\sum C_{\beta \gamma} q^{\beta}|\hat{k}-\mathbf{q}|^{\gamma}$. Clearly, the basic integral involved is

$$
C(\alpha, \beta, d) \equiv \text { Finite part of } \int d^{d} q \frac{1}{|\hat{k}-\mathbf{q}|^{\alpha}} \frac{1}{q^{\beta}}
$$

which can be evaluated via the standard method. We find

$$
C(\alpha, \beta, d)=\frac{1}{2} \frac{\Gamma\left(\frac{d}{2}\right) \Gamma\left(\frac{\alpha+\beta}{2}-\frac{d}{2}\right) \Gamma\left(\frac{d}{2}-\frac{\beta}{2}\right) \Gamma\left(\frac{d}{2}-\frac{\alpha}{2}\right)}{\Gamma\left(\frac{\alpha}{2}\right) \Gamma\left(\frac{\beta}{2}\right) \Gamma\left(d-\frac{\alpha+\beta}{2}\right)} .
$$

Substituting $b(\hat{k}, \mathbf{q})$, in the form of $\sum C_{\beta \gamma} q^{\beta}|\hat{k}-\mathbf{q}|^{\gamma}$, back to $\Gamma_{b}$, and after some 
lengthy but straightforward algebra, we find

$$
\begin{aligned}
& \Gamma_{b}(\Delta, z, d)=-\frac{d-1}{8} C(\Delta+2, z, d)+\frac{d-1}{4} C(\Delta+2, z-2, d)- \\
& \frac{d-1}{8} C(\Delta+2, z-4, d)-\frac{d+1}{8} C(\Delta-2, z, d)+\frac{1}{8} C(\Delta, z+2, d)+ \\
& \frac{1}{8} C(\Delta, z-4, d)-\frac{1}{8} C(\Delta-6, z+2, d)+\frac{2 d-3}{8} C(\Delta, z-2, d)+ \\
& \frac{3}{8} C(\Delta-4, z+2, d)+\frac{2 d-3}{8} C(\Delta, z, d)-\frac{3}{8} C(\Delta-2, z+2, d)+ \\
& \frac{3}{8} C(\Delta-4, z, d)-\frac{3}{8} C(\Delta-2, z-2, d) .
\end{aligned}
$$

The above expression is ready for direct computer computations. We find that $\Delta=$ $d+\frac{1}{2}$ is the only solution of (B.19) persistent in all dimensions. In addition to this solution, there are two other solutions at $d=2: \Delta=3$ and $\Delta=\frac{7}{2}$. It is interesting to note that the solution, $\Delta=\frac{7}{2}$, could also be obtained by assuming that the enstrophy flux is constant over the inertial range. These solutions disappear when $|d-2| \gtrsim 0.05$. Therefore, they exist essentially at $d=2$.

We now go back to the old parametrization of $\eta(s)=s^{6-d-y}$ and ask how the $y$-expansion solutions, $z=2-\frac{y}{4}$ and $\Delta=d-2+\frac{y}{2}$, connect to the solutions, $z=1$ and $\Delta=d+\frac{1}{2}$.

For this end, we first include $\eta(s)$ in (B.16)

$$
\begin{aligned}
& \frac{u(s)}{|g(s)|^{2}}=D s^{\Delta-2 z+6-d-y}+ \\
& h_{1}{ }^{2} h_{2}\left(m_{0}\right)^{d+2-\Delta-2 z}\left[\Gamma_{a}(\Delta, \Delta, d) s^{d+2-\Delta-2 z}+u_{\infty} s^{2-2 z} u(s)+\cdots\right]
\end{aligned}
$$




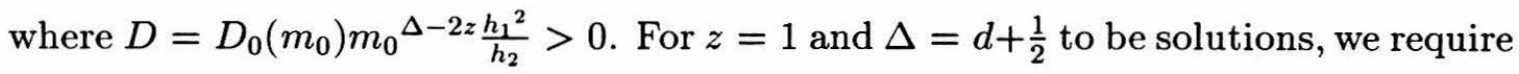
$s^{d+2-\Delta-z}$ be the the next-to-leading order term, and thus $\Delta-2 z+6-d-y<d-\Delta<0$. Clearly, this implies that $z=1$ and $\Delta=d+\frac{1}{2}$ are valid only when $y>5$. On the other hand, because the $y$-expansion solutions are obtained under the condition that both $s^{\Delta-2 z+6-d-y}$ and $s^{d+2-\Delta-2 z}$ are leading terms, and this is true only when $s^{2-2 z}$ is subleading, hence $y$ has to be less than 4 . As a result, when $4 \leq y \leq 5$, none of them is the solution.

One must bear in mind that although (B.16) becomes (B.20) when the force is present, (B.15) is unchanged and will always fix $z$ to 1 when $\Delta>d$ (or $y>4$ ). Therefore, $s^{2-2 z}$ is always the leading term in (B.20) when $y>4$. As a consequence, when $4<y<5$, solutions are obtained by setting both $s^{\Delta-2 z+6-d-y}$ and $s^{d+2-\Delta-2 z}$ as the next-to-leading order terms. We get $z=1, \Delta=d-2+y / 2$ and $g_{1}=-\frac{\Gamma_{b}}{2 u_{\infty}}=$ $-\frac{\Gamma_{a}}{2 u_{\infty}}-\frac{D}{2}$. Hence, for $4 \leq y \leq 5$, the self-consistent equation is

$$
\Gamma_{b}-\Gamma_{a}=u_{\infty} D
$$

The implication of (B.21) for numerical computations of the exponent $\Delta$ is clear. If one starts from $y=4$, then one should look for at what value of $y$ the difference between $\Gamma_{a}$ and $\Gamma_{b}$ vanishes. Suppose this happens at $y_{c}$, then at $y_{c}$ and beyond $y_{c}$, $\Delta$ sticks to $d-2+y_{c} / 2$. 


\section{Appendix C}

\section{Details of Numerical Work}

In this Appendix, we outline our numerical work for $u(s)$ and $y_{c}$. We start from the subtracted version of $(4.11)$

$$
-u(s) \operatorname{Re}\left[\Delta J_{b}(s)\right]-\sqrt{1-\frac{s^{2}}{4}} \Delta J_{a}(s)-\sqrt{1-\frac{s^{2}}{4}} \delta_{0}=0,
$$

where

$$
\begin{aligned}
-\operatorname{Re}\left[\Delta J_{b}(s)\right]= & \int_{\mathbf{q}, t} u\left(\frac{s-q^{z} t}{|\hat{k}-\mathbf{q}|^{z}}\right)|\hat{k}-\mathbf{q}|^{-\Delta} . \\
& {\left[b(\hat{k}, \mathbf{q}) \sqrt{1-\frac{t^{2}}{4}}-\tilde{b}\left(\hat{k}, \frac{\hat{k}-\mathbf{q}}{|\hat{k}-\mathbf{q}|}\right) \sqrt{1-\frac{s^{2}}{4}}\right], } \\
\Delta J_{a}(s)= & \int_{\mathbf{q}, t} u\left(\frac{s-q^{z} t}{|\hat{k}-\mathbf{q}|^{z}}\right)|\hat{k}-\mathbf{q}|^{-\Delta} . \\
& {\left[b(\hat{k}, \mathbf{q}) u(t) q^{z-\Delta}-\tilde{b}\left(\hat{k}, \frac{\hat{k}-\mathbf{q}}{|\hat{k}-\mathbf{q}|}\right) u(s)\right] }
\end{aligned}
$$

We emphasize that in (C.1) the integrals are over $|t|<2$ and $|(s-q t) /| \hat{k}-\mathbf{q}||<2$. For convenience, we scale down a factor 2 by setting $s^{\prime}=s / 2, t^{\prime}=t / 2$, and $\tilde{u}(x) \equiv u(2 x)$. 
Then, for example, we may write

$$
\begin{aligned}
& \int_{\mathbf{q}} \int_{-2}^{2} d t b(\hat{k}, \mathbf{q}) \sqrt{1-\frac{t^{2}}{4}}|\hat{k}-\mathbf{q}|^{-\Delta} u\left(\frac{s-q t}{|\hat{k}-\mathbf{q}|}\right) \\
& =2 A_{d-1} \int_{-1}^{1} d t^{\prime} \int_{0}^{\infty} d q \int_{-1}^{1} d y\left(1-y^{2}\right)^{\frac{d-3}{2}} q^{d-1} \frac{b(q, y) \sqrt{1-t^{\prime 2}}}{\left(1-2 q y+q^{2}\right)^{\frac{\Delta}{2}}} \tilde{u}\left(\frac{s^{\prime}-q t^{\prime}}{\sqrt{1-2 q y+q^{2}}}\right) \\
& \equiv 2 A_{d-1} I\left(s^{\prime}\right)
\end{aligned}
$$

with $A_{d-1}=$ area of unit sphere in $d-1$ dimension.

We convert the infinite integral domain of $q$ into a finite one by performing a conformal transformation $q \rightarrow 1 / q$ on $\int_{1}^{\infty} d q$ and find that

$$
\begin{aligned}
I(s)= & \int_{-1}^{1} d t \int_{0}^{1} d q \int_{-1}^{1} d y \sqrt{1-t^{2}}\left[K_{d}^{(1)}(q, y) \tilde{u}\left(\frac{s-q t}{\sqrt{1-2 q y+q^{2}}}\right)+\right. \\
& \left.K_{d}^{(2)}(q, y) \tilde{u}\left(\frac{t-q s}{\sqrt{1-2 q y+q^{2}}}\right)\right]
\end{aligned}
$$

where we have defined

$$
\begin{aligned}
& K_{d}^{(1)}(q, y)=\left(1-y^{2}\right)^{\frac{d-3}{2}} \frac{b(q, y)}{\left(1-2 q y+q^{2}\right)^{\frac{\Delta}{2}}} q^{d-1} \\
& K_{d}^{(2)}(q, y)=\left(1-y^{2}\right)^{\frac{d-3}{2}} \frac{b(q, y)}{\left(1-2 q y+q^{2}\right)^{\frac{\Delta}{2}}} q^{\Delta-d-3} .
\end{aligned}
$$

The singular point is now located at $y=1$ and $q=1$, but is subtracted. We may also rewrite the other integrals in the same way, then (C.1) becomes

$$
\tilde{u}(s) B(s)-\sqrt{1-s^{2}} A(s)-\sqrt{1-s^{2}} \tilde{\delta}_{0}=0 .
$$


Here $\tilde{\delta}_{0}=\delta_{0}\left(2 A_{d-1}\right)^{-1}$ and we have defined

$$
\begin{aligned}
& A(s)= \int_{-1}^{1} d t \int_{0}^{1} d q \int_{-1}^{1} d y \tilde{u}\left(\frac{s-q t}{\sqrt{1-2 q y+q^{2}}}\right)\left[K^{(2)}(q, y) \tilde{u}(t)-K_{d}^{(3)}(q, y) \tilde{u}(s)\right]+ \\
& \tilde{u}\left(\frac{t-q s}{\sqrt{1-2 q y+q^{2}}}\right)\left[K^{(1)}(q, y) \tilde{u}(t)-K_{d}^{(4)}(q, y) \tilde{u}(s)\right] \\
& B(s)= \int_{-1}^{1} d t \int_{0}^{1} d q \int_{-1}^{1} d y \tilde{u}\left(\frac{s-q t}{\sqrt{1-2 q y+q^{2}}}\right)\left[K_{d}^{(1)}(q, y) \sqrt{1-t^{2}}-\right. \\
&\left.K_{d}^{(3)}(q, y) \sqrt{1-s^{2}}\right]+\tilde{u}\left(\frac{t-q s}{\sqrt{1-2 q y+q^{2}}}\right)\left[K_{d}^{(2)}(q, y) \sqrt{1-t^{2}}-K_{d}^{(4)}(q, y) \sqrt{1-s^{2}}\right]
\end{aligned}
$$

and

$$
\begin{aligned}
& K_{d}^{(3)}(q, y)=\left(1-y^{2}\right)^{\frac{d-3}{2}} \frac{\tilde{b}(q, y)}{\left(1-2 q y+q^{2}\right)^{\frac{\Delta}{2}}} q^{d-1}, \\
& K_{d}^{(4)}(q, y)=\left(1-y^{2}\right)^{\frac{d-3}{2}} \frac{\tilde{b}(q, y)}{\left(1-2 q y+q^{2}\right)^{\frac{\Delta}{2}}} q^{\Delta-d-3}, \\
& K^{(1)}(q, y)=\left(1-y^{2}\right)^{\frac{d-3}{2}} \frac{b(q, y)}{\left(1-2 q y+q^{2}\right)^{\frac{\Delta}{2}}} q^{2 \Delta-d-4}, \\
& K^{(2)}(q, y)=\left(1-y^{2}\right)^{\frac{d-3}{2}} \frac{b(q, y)}{\left(1-2 q y+q^{2}\right)^{\frac{\Delta}{2}}} q^{d-\Delta},
\end{aligned}
$$

with $\tilde{b}(q, y) \equiv \frac{q^{2}\left(1-y^{2}\right)}{1-2 q y+q^{2}}$.

The integration is done by Gaussian quadrature using Gauss-Legendre weights and points. Thus $\int_{-1}^{1} d t \int_{0}^{1} d q \int_{-1}^{1} d y F(s, t, q, y)$ is replaced by

$$
\sum_{i=1}^{n} \sum_{j=n_{1}}^{n} \sum_{k=1}^{n} w(i) w(j) w(k) F(x(l), x(i), x(j), x(k))
$$

where $w(i)$ are the weights, $x(i)$ reside on the Gauss-Legendre points of $(-1,1)$, and 
$n, n_{1}$ are taken to be 50,26 , considering the capacity of our computer system (Sun SPARC $1+$ station).

As a result of discretization, (C.2) becomes a system of nonlinear equations which then may be fed into any "standard" subroutine designed for such a problem. Our error bar, when finding the root, is always at most $O\left(10^{-12}\right)$ for each individual equation.

The interpolation scheme is linear. For comparison, we also run a computation at $\Delta=d+\frac{3}{2}$ and $d=3$, using a quadratic interpolation. The difference is very tiny as shown in Figure C-1. Increasing divisions to 100 also has little effect (see Figure C-2). It is important to note that (C.1) is homogeneous in $u(s)$. Hence, if $u(s)$ is a solution, so is $\lambda u(s)$ for any $\lambda$. Similarly, if $\tilde{u}(s)$ and $\tilde{\delta}_{0}$ are solutions to (C.2), so are $\lambda \tilde{u}(s)$ and $\lambda \tilde{\delta}_{0}$. Numerically, it is convenient to fix the value of $\tilde{u}(s)$ at some particular $s$. (In our work, we normalize $\tilde{u}(0)$ to 1 .) Let such a solution be $\tilde{u}_{p}(s)$, then the general solution is $\lambda \tilde{u}_{p}(s)$. Consequently, we have

$$
U(\mathbf{k}, \omega)=\frac{A_{2}}{k^{\Delta}}\left[\lambda \tilde{u}_{p}\left(\frac{w}{2 \tilde{\nu} k^{z}}\right)+u_{1}\left(\frac{w}{2 \tilde{\nu} k^{z}}\right) x^{d+1-\Delta}+\cdots\right]
$$

Physically, $\lambda$ is fixed by $u_{0}$. To see it, we use the expression

$$
u_{0}=\frac{d-1}{d} \frac{m_{0}^{\Delta-d-1}}{A_{2} \tilde{\nu}} \int_{\mathbf{k} \omega} U(\mathbf{k}, \omega)
$$




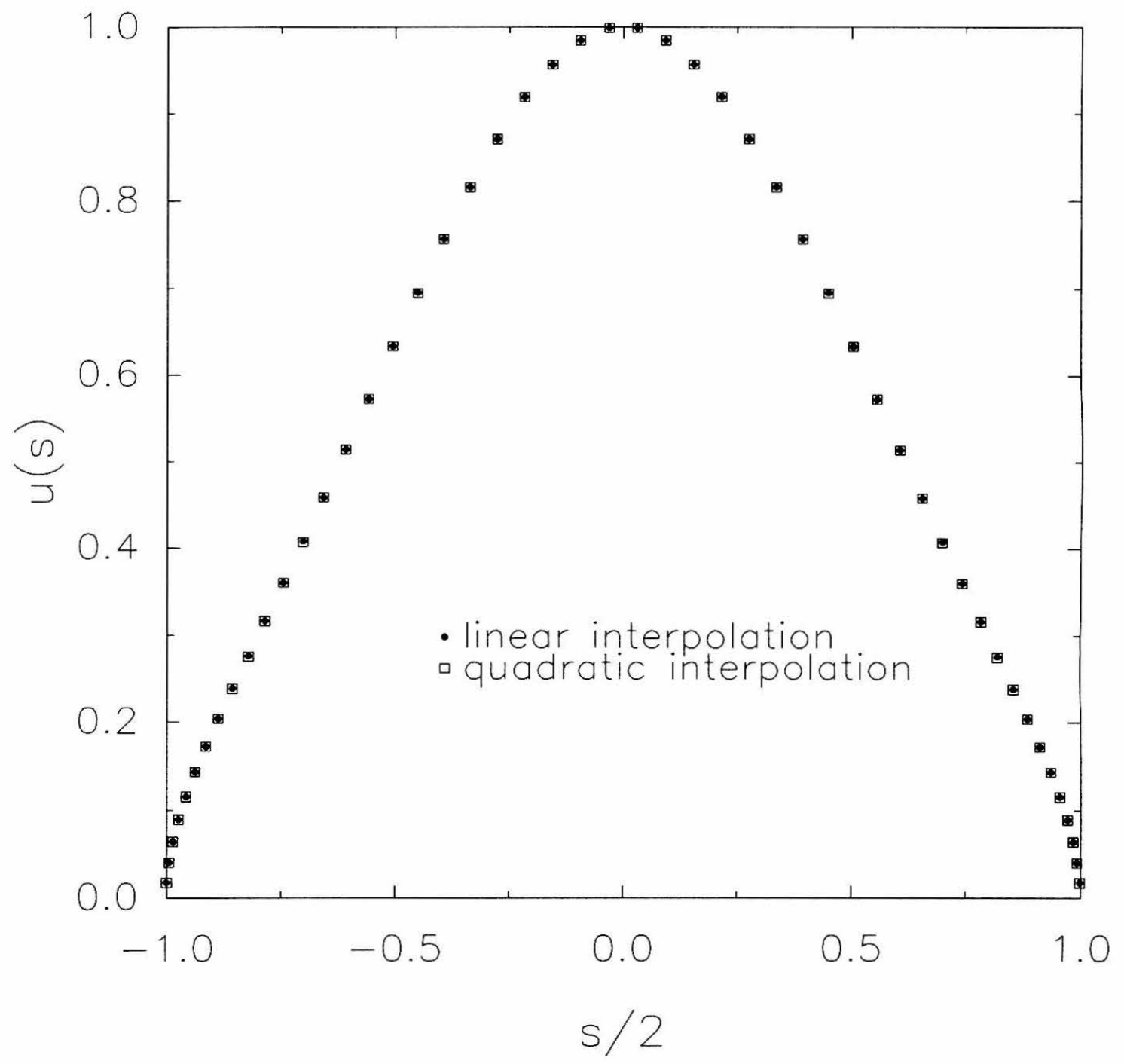

Figure C-1: Comparison of linear and quadratic interpolations 


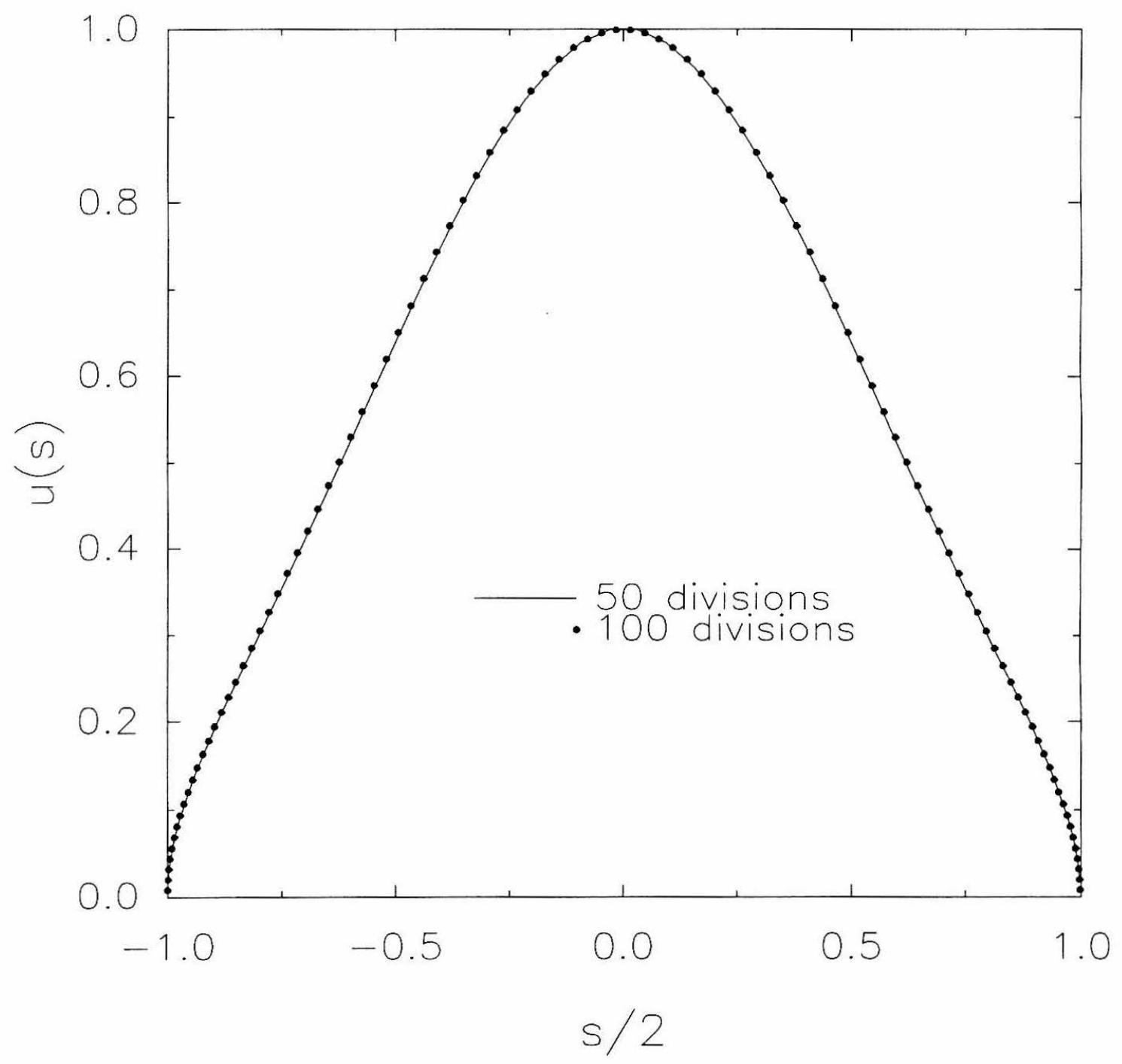

Figure C-2: Comparison of two different numbers of divisions 


\section{4}

Assuming that $\int_{k<m_{0} w} U(k, w)$ is negligible when $m_{0} \rightarrow 0$, we have

$$
u_{0}=4 \lambda \frac{d-1}{d} K_{d} \int_{-1}^{1} \frac{d x}{2 \pi} \tilde{u}_{p}(x)
$$

where $(2 \pi)^{d} K_{d}$ is the area of unit sphere in $d$ dimension and we have made use of (C.3). Finally, we have

$$
\lambda=\frac{d u_{0}}{4 K_{d}(d-1) \int_{-1}^{1} \frac{d x}{2 \pi} \tilde{u}_{p}(x)} .
$$




\section{Appendix D}

\section{Large- $N$ Limit of a Cubic Invariant}

In this Appendix, we describe briefly the large- $N$ limit of a model with the following cubic trace invariant

$$
\begin{aligned}
A_{N}^{l m n}= & f(N) \tilde{\delta}(l+m+n) \times \\
& {\left[e^{\frac{2 \pi i}{M} l \times m}+e^{-\frac{2 \pi i}{M} l \times m}+e^{\frac{2 \pi i}{M} m \times n}+e^{-\frac{2 \pi i}{M} m \times n}+e^{\frac{2 \pi i}{M} n \times l}+e^{-\frac{2 \pi i}{M} n \times l}\right], }
\end{aligned}
$$

where the notations will be explained shortly. We shall show that this model selects all of the diagrams in the large- $N$ limit and therefore is not useful in considering the large- $N$ expansion of the exponents.

In (D.1), following the trace invariants approach described in Section $2.1, A^{l m n}$ is constructed from $\mathrm{SU}(\mathrm{M})$, where $M$ is an odd integer. $l, m$, and $n$ are two-dimensional vectors represented by $\left(a_{x}, a_{y}\right)$, where $a_{x}$ and $a_{y}$ are integers which may be confined 
in

$$
-\frac{M-1}{2} \leq a_{x}, a_{y} \leq \frac{M-1}{2}
$$

with the origin $(0,0)$ excluded. Hence the number of allowed $\left(a_{x}, a_{y}\right)$ is $N \equiv M^{2}-1$. $\tilde{\delta}(a)$ is 1 if $\left(a_{x} \bmod M, a_{y} \bmod M\right)=(0,0) . f(N)$ is introduced for the purpose of normalization and will be chosen below. Clearly, $A_{N}^{l m n}$ is periodic in any of the indices $l, m, n$ with the period $M$ in both $x$ and $y$ directions. Thus the choice of (D.2) is only a convention.

The generators $\mathbf{J}^{l}, l=1, \cdots, N$, which define the cubic trace invariant $A_{N}^{l m n}(\sim$ $\left.\operatorname{tr}\left(\mathbf{J}^{l}\left\{\mathbf{J}^{m}, \mathbf{J}^{n}\right\}\right)\right)$, are a special basis of $\operatorname{su}(\mathrm{M})$ algebra, which is also useful in constructing finite modes approximation of 2D Euler's equation[35]. We refer readers to references[36] for their explicit matrix representations. Here, for our purpose, knowing their explicit forms is not necessary.

In this model, $\mathbf{v}^{l}$ transforms according to $\mathrm{SU}(\mathrm{M})$ of which the dimension of representation is $N$ (see (2.41)). It can be shown that there always exists an irreducible representation of $\mathrm{SU}(\mathrm{M})$ with the dimension of representation equal to $N$. (This representation is associated with the Young diagram in which the first row has two columns, while the rest $M-1$ rows have one column.) Hence rules (i) and (ii) of Chapter 3 are valid here.

As before, the derivation of large- $N$ is proceeded first by computing

$$
I_{2}=\sum_{l, m, n}\left(A_{N}^{l m n}\right)^{2}
$$


which can be reduced to

$$
[3 f(N)]^{2} \sum_{m, n}\left[e^{\frac{2 \pi i}{M} m \times n}+e^{-\frac{2 \pi i}{M} m \times n}\right]^{2} .
$$

There are two types of terms when we expand out the bracket in the above equation. One is the cross term which has a zero phase and is proportional to $N^{2}$ after summing over $n$ and $m$. The other terms, $e^{ \pm \frac{4 \times i}{M} m \times n}$, have non-zero phases. Summing of these terms over $n$ and $m$ may be evaluated first by computing more generally the following sum

$$
S_{a} \equiv \sum_{n} e^{\frac{2 \pi i}{M} n \times a} ; \quad a=\left(a_{x}, a_{y}\right)
$$

where $a_{x}$ and $a_{y}$ are integers. In the large- $N$ limit (hence $M$ is also large), $S_{a}$ may be estimated by

$$
\begin{aligned}
\frac{S_{a}}{M^{2}} & =\int_{-\frac{1}{2}}^{\frac{1}{2}} d x \int_{-\frac{1}{2}}^{\frac{1}{2}} d y e^{i 2 \pi\left(x a_{y}-y a_{x}\right)}+O\left(\frac{1}{M^{2}}\right) \\
& =\frac{\sin \pi a_{x}}{\pi a_{x}} \frac{\sin \pi a_{y}}{\pi a_{y}}+O\left(\frac{1}{M^{2}}\right),
\end{aligned}
$$

where the summations over $n_{x}$ and $n_{y}$ are replaced by the integrals over $x=n_{x} / M$ and $y=n_{y} / M$. Clearly, if $a_{x}$ and $a_{y}$ are integers but not equal to multiples of $M$, then the integral vanishes and hence $S_{a} \sim O(1)$. On the other hand, when $\left(a_{x} \bmod M\right.$, $\left.a_{y} \bmod M\right)=(0,0), S_{a}=N$. This implies

$$
S_{a}=N \tilde{\delta}(a)+O(1) .
$$


The most important consequence of (D.3) is that terms with non-zero phases are subdominant to terms with zero phase. For example, in the evaluation of $I_{2}$, the zero phase term is $O\left(N^{2}\right)$, while the non-zero phase terms are only $O(N)$. This conclusion applies equally well to the evaluations of other 2PI diagrams. Consequently, the leading order terms for any $2 \mathrm{PI}$ diagrams must be these with zero phase. In the rest of this Appendix, we shall lay out an argument to establish the existence of such terms by explicit constructions. Following this argument, we give a simple example to demonstrate the constructions. Then, we show that because of the existence of zero phase terms in any 2PI diagrams, all of the 2PI diagrams are $O(N)$.

For any 2PI diagram, we assign an index to each line obeying the "conservation" of indices at each vertex (see Figure D-1). The conservation of indices at each vertex originates from the $\tilde{\delta}$ function in (D.1). There are two possible configurations at each vertex, i.e, either two indices coming out from the vertex (Figure D-1(a)) or two indices combined at the vertex (Figure D-1(b)). In both cases, $l$ may be substituted in terms of $m$ and $n ; A_{N}^{l m n}$ then becomes

$$
A_{N}^{l m n}=3 f(N) \tilde{\delta}(l+m+n)\left[e^{\frac{2 \pi i}{M} m \times n}+e^{\frac{2 \pi i}{M} n \times m}\right] .
$$

Therefore, each vertex contributes either $e^{\frac{2 \pi i}{M} m \times n}$ or $e^{\frac{2 \pi i}{M} n \times m}$. The rules for the construction of a term with zero phase are to choose the cross product $(m \times n$ or $n \times m)$ clockwise at each vertex. (Counterclockwise is equally good as long as one sticks to one of the conventions all the time.) For example, if our convention is clockwise, then 


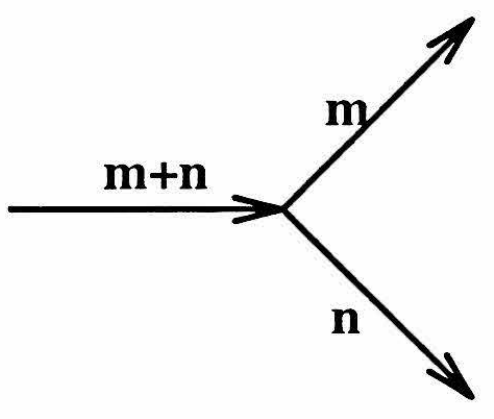

(a)
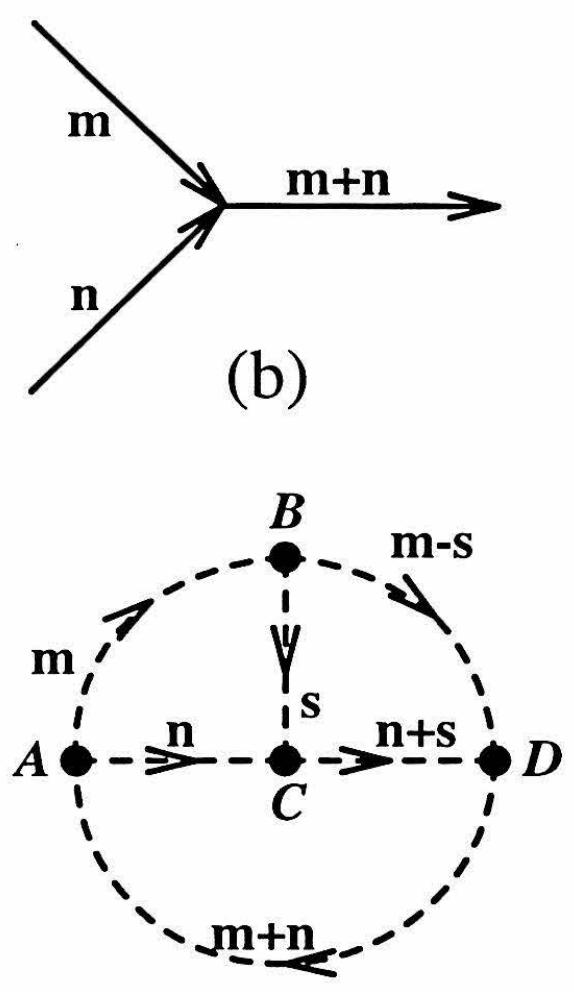

(c)

Figure D-1: Two possible configurations: (a) $m \times n$ (b) $n \times m$ (c) An example for the construction of a zero-phase term 
Figure D-1(a) contributes $e^{\frac{2 \pi i}{M} m \times n}$, while Figure D-1(b) contributes $e^{\frac{2 \pi i}{M} n \times m}$. Obviously, if two indices $m$ and $n$ depart from a vertex somewhere in the diagram, they will eventually combine at another vertex. Therefore, according to our rules, the phases due to $m$ and $n$ at these two vertices are canceled $(m \times n+n \times m=0)$. This concludes our proof for the existence of zero-phase terms.

As an example, we consider the diagram shown in Figure D-1(c), which is $I_{4}$ according the notations of Chapter 3. By using (D.4), its explicit expression is

$$
I_{4}=[3 f(N)]^{4} \sum_{m, n, s} B_{m, n} B_{m-s, s} B_{n, s} B_{n+s, m-s}
$$

where $B_{m, n} \equiv e^{\frac{2 \pi i}{M} m \times n}+e^{\frac{2 \pi i}{M} n \times m}$. Note that the contributions of vertices $A, B, C$, and $D$ are $B_{m, n}, B_{m-s, s}, B_{n, s}, B_{n+s, m-s}$ respectively. Our rules correspond to the case when $B_{m, n}, B_{m-s, s}, B_{n, s}$, and $B_{n+s, m-s}$ contribute $e^{\frac{2 \pi i}{M} m \times n}, e^{\frac{2 \pi i}{M}(m-s) \times s}, e^{\frac{2 \pi i}{M} n \times s}$, $e^{\frac{2 \pi i}{M}(n+s) \times(m-s)}$ respectively. One can easily check that this gives a null phase.

Since there are three free dummy indices, $I_{4} \sim(3 f)^{4} N^{3}$. In general, if a 2PI diagram has $2 k$ vertices, there are $k+1$ free dummy indices. Hence we conclude $I_{2 k}^{(i)} \sim(3 f)^{2 k} N^{k+1}$. As a result, one may choose $(3 f)^{2} N=1$, i.e., $f=\frac{1}{3 \sqrt{N}}$. Under this normalization, $I_{2 k}^{(i)}=O(N) \equiv O\left(N^{1-\alpha_{k}^{i}}\right)$. This gives $\alpha_{k}^{i}=0$ and hence all of the 2 PI diagrams survive as $N \rightarrow \infty$. 


\section{References}

[1] (a) A. N. Kolmogorov, C. R. Acad. Sci. U.S.S.R. 30, 301 (1941); 32, 16 (1941).

(b) L.D. Landau and E.M. Lifshitz, Fluid Mechanics (2nd Edition, Pergamon, 1987), Chap. 3.

[2] See, e.g., A. Monin and A. M. Yaglom, Statistical Fluid Mechanics, Vol. 2 (MIT Press, Cambridge, 1975).

[3] See, e.g., T. S. Lundgren, Phys. Fluids 25, 2193 (1982); L'vov, Phys. Rep. 207, 1 (1991); Yakhot and Zakharov, unpublished.

[4] (a) D. Forster, D. R. Nelson, and M. J. Stephen, Phys. Rev. A 16,732 (1977).

(b) C. DeDominicis and P. C. Martin, Phys. Rev. A 19, 419 (1979).

[5] Such coincidences are not so uncommon in critical phenomena: consider, for example, the proximity of the critical correlation decay exponent, $\eta$, to the mean field value $\eta=0$. Also, the specific heat exponent, $\alpha$, for the superfluid transition in ${ }^{4} \mathrm{He}$ is very nearly zero, and the susceptibility exponent, $\gamma$, for the $3-d$ Ising model is very close to $5 / 4$. One knows in these cases that exponents are not simply rational numbers, and there is no simple way of obtaining their values. 
[6] See, e.g., P. Manneville, Dissipative Structures and Weak Turbulence (Academic Press, New York, 1990).

[7] See, e.g., V. Yakhot and S. A. Orszag, J. Sci. Comput. 1, 3 (1986).

[8] This analogy, as far as we know, originates from an oral presentation by P. C. Hohenberg, and was brought into our attention by M. C. Cross (private communication).

[9] M. E. Fisher, S. -K. Ma, and B. G. Nickel, Phys. Rev. Lett. 29, 917 (1972).

[10] See, e.g., K.G. Wilson and J. Kogut, Phys. Rep. 12C, 75 (1974).

[11] J. Sak, Phys. Rev. B 8, 281 (1973); 15, 4344 (1977).

[12] R. Abe, Prog. Theor. Phys. 49,113 (1973); S.-K. Ma, Phys. Rev. A 7, 2172 (1973).

[13] Results for this thesis have been reported briefly in C. -Y. Mou and P. B. Weichman, Phys. Rev. Lett. 70, 1101 (1993).

[14] R. H. Kraichnan, J. Fluid Mech. 5, 497 (1959).

[15] See, e.g., R. H. Kraichnan, Phys. Fluids 8, 575 (1965); L'vov, Phys. Rep. 207, $1(1991)$.

[16] D. R. Nelson and E. Domany, Phys. Rev. B 13, 236 (1976). 
[17] Kraichnan has considered equations of the form (2.1) in which $A_{N}^{l m n}$ is randomly $\pm 1 / N$, subject only to symmetry under any permutation of indices. In the limit $N \rightarrow \infty$ this model yields precisely the results we obtain: the DIA equations become exact. For a review, see R. H. Kraichnan and S. Chen, Physica D 37, 160 (1989). However, if one contemplates a calculation of higher order corrections due to finite $N$, only when the generalized fluid equations include some higher symmetry do we expect the universal exponents to vary continuously with $N$, thereby yielding a systematic expansion. We do not expect Kraichnan's model to respect this requirement.

[18] We could further compactify the notation, with a general relativistic flavor, using upper and lower indices on $\stackrel{D}{D}_{N}$ and defining $D^{l}{ }^{\prime}(g)=D^{l^{\prime}}{ }_{l}\left(g^{-1}\right)$, but we wish to spare the reader the added confusion.

[19] P. C. Martin, E. Siggia, and H. Rose, Phys. Rev. A 8, 423 (1973).

[20] H. Wyld, Ann. Phys. (N.Y.) 14, 143 (1961).

[21] See, e.g., J. Zinn-Justin, Quantum Field Theory and Critical Phenomena (Clarendon Press, Oxford, 1989), Chap. 16.

[22] See, e.g., L.D. Landau and E.M. Lifshitz, Quantum Mechanics (3rd Edition, Pergamon, 1977), Chaps. 4, 8, and 14.

[23] (a) H. Georgi, Lie Algebra in Particle Physics (Benjamin Cummings, Reading, Mass., 1982). 
(b) D. B. Lichtenberg, Unitary Symmetry and Elementary Particles (2nd Edition, Academic Press, New York, 1978).

[24] D. J. Amit and D. V. I. Roginsky, J. Phys. A 12, 689 (1979), note that the rules listed in Figure 3-1 generalize what were used by the above authors.

[25] R. H. Kraichnan, J. Math. Phys. 2, 124 (1961).

[26] U. Frisch, M. Lesieur, and A. Brissaud, J. Fluid Mech. 65, 145 (1974).

[27] We thank D. R. Nelson for pointing this out to us.

[28] See, e.g., C. W. Van Atta and W. Y. Chen, J. Fluid Mech. 44, 145, 1970;

F. Anselmet, F. Gagne, and E. J. Hopfinger, J. Fluid Mech. 140, 63, 1984.

[29] M. Kardar, G. Parisi, and Y.-C. Zhang, Phys. Rev. Lett. 56, 889 (1986).

[30] G. T. Taylor, Proc. Roy. Soc. A164, No.919, 476 (1938).

[31] See, e.g., H. Tennekes, J. Fluid Mech. 67, 561 (1975); M. Nelkin and M. Tabor, Phys. Fluids A2, 81 (1990).

[32] As it turns out that $\epsilon \equiv \Delta-d=1 / 2$, any integers are thus multiples of $\epsilon$. The complete expansions are then $u(s)=1+\sum_{n=1}^{\infty} u_{n} s^{-n \epsilon}, g(s)=1+\sum_{n=1}^{\infty} g_{n} s^{-n \epsilon}$. In principle, one can work out each term in the expansions.

[33] E. A. Kuznetsov and V. S. L'vov, Physica 2D, 203 (1981). 
[34] A careful treatment would involve introducing a small cutoff around $\hat{k}$. Since $\hat{k}$ is not changed under the conformal transformation, this will not change the conclusion.

[35] See, e.g., J. Miller, P. B. Weichman, and M. C. Cross, Phys. Rev. A 45, 2328 (1992); J. S. Dowker and A. Wolski, Phys. Rev. A 46, 6417 (1992).

[36] G. 't Hooft, Nucl. Phys. B 138, 1 (1978); D. B. Fairlie and Z. K. Zachos, Phys. Lett. B 224, 101 (1989).

[37] See, e.g., C. W. Van Atta and J. C. Wyngaard, J. Fluid Mech. 72, 673 (1975). 